\title{
The cultural evolution of emergent group-level traits
}

\author{
Paul E. Smaldino \\ Center for Advanced Modeling in the Social, Behavioral, and Health Sciences, \\ Johns Hopkins University, Baltimore, MD 21209. \\ paul.smaldino@gmail.com \\ http://www.smaldino.com
}

\begin{abstract}
Many of the most important properties of human groups - including properties that may give one group an evolutionary advantage over another - are properly defined only at the level of group organization. Yet at present, most work on the evolution of culture has focused solely on the transmission of individual-level traits. I propose a conceptual extension of the theory of cultural evolution, particularly related to the evolutionary competition between cultural groups. The key concept in this extension is the emergent group-level trait. This type of trait is characterized by the structured organization of differentiated individuals and constitutes a unit of selection that is qualitatively different from selection on groups as defined by traditional multilevel selection (MLS) theory. As a corollary, I argue that the traditional focus on cooperation as the defining feature of human societies has missed an essential feature of cooperative groups. Traditional models of cooperation assume that interacting with one cooperator is equivalent to interacting with any other. However, human groups involve differential roles, meaning that receiving aid from one individual is often preferred to receiving aid from another. In this target article, I discuss the emergence and evolution of group-level traits and the implications for the theory of cultural evolution, including ramifications for the evolution of human cooperation, technology, and cultural institutions, and for the equivalency of multilevel selection and inclusive fitness approaches.
\end{abstract}

Keywords: altruism; cooperation; cultural group selection; inclusive fitness; innovation; institutions; interdependence; multilevel selection; niche construction

\section{Introduction}

Humans are social animals, creatures of culture. Cultural traits are transmitted across generations, and varieties of socially learned norms and behaviors compete in a manner that is analogous to Darwinian natural selection. Over the last few decades, a theory of cultural evolution has arisen to describe the transmission of traits via social learning rather than genetic inheritance, as well as the ways in which genes and cultural traits coevolve (Boyd \& Richerson 1985; Cavalli-Sforza \& Feldman 1981; Durham 1991; Mesoudi 2011; Richerson \& Boyd 2005; Whiten et al. 2011). Despite the claims of some who doubt the applicability of evolutionary models to cultural evolution (e.g., Claidière \& André 2012; Fracchia \& Lewontin 1999; Pinker 2012) and despite the real need for better, more explanatory models specific to cultural evolution (Mesoudi 2007; Sperber \& Claidière 2006), an evolutionary theory of culture is here to stay. Cultural knowledge and behaviors are transmitted from generation to generation, and improved ideas and practices replace those that are less effective. Formal cultural evolutionary theory has gained additional robustness from models that show that natural selection can operate on cultural variants even if traits are not discrete and even if transmission is highly error-prone (Henrich et al. 2008).

The development of a cultural evolutionary theory, however, has suffered from an overemphasis on the experiences and behaviors of individuals at the expense of acknowledging complex group organization and behavior. For example, Richerson and Boyd (2005), two of the seminal figures in the development of a formal theory of cultural evolution, have defined culture as "information capable of affecting individuals' behavior that they acquire from other members of their species through teaching, imitation, and other forms of social transmission" (p. 5, italics added). Cultures, however, are more than collections of individuals with shared traits. Cultural groups are organized, and organization matters. I argue that many important behaviors related to the success and function of human societies are only properly defined at the level of groups. I further argue that group-level traits, which involve organized collections of differentiated individuals, present a unit of cultural selection that is not encompassed by selection on individuals. This implies that selection on group-level traits is qualitatively different

Paul E. Smaldino is a Postdoctoral Fellow at the Center for Advanced Modeling in the Social, Behavioral, and Health Sciences at Johns Hopkins University. He was educated at Wesleyan University (B.A. in Physics) and the New School for Social Research (M.A. in Psychology) before receiving his Ph.D. in Psychobiology from the University of California, Davis. His research focuses on how organizational patterns emerge in humans and other social organisms, with perspectives drawn from psychology, evolutionary ecology, and the social sciences. He is particularly interested in the use of computer simulations to uncover principles of social dynamics and evolution. 
from selection on groups as defined by traditional multilevel selection (MLS) theory, which does not account for emergent traits based on group organization. A further implication is that a fully fleshed out multilevel selection theory of cultural evolution cannot be reduced to an inclusive fitness approach that focuses solely on the individual, as some have argued (see discussion in Pinker 2012).

In this target article, I will discuss the significance of what I term group-level traits in the context of human cultural evolution. I will first describe what I mean by grouplevel traits. Second, I will show that this type of organization is not fully accounted for by the multilevel selection perspective currently in vogue to describe evolutionary competition between cultural groups. Third, I will extend this analysis to show that cooperation is insufficient as the defining feature of humans groups and that in many contexts collaborative interdependence is more appropriate. Fourth, I will discuss how group-level traits emerge from collections of individual actors. Fifth, I will discuss mechanisms by which group-level traits are maintained, transmitted, and evolve. Sixth, I will argue that the interactional complexity of human societies supports extending the perspective of multilevel selection to incorporate the causal interactions between individuals and groups and that this extended perspective eliminates the equivalence between multilevel selection and inclusive fitness approaches to modeling evolution. Finally, I will suggest directions for future research and theoretical development to better understand the evolution of group-level traits.

\section{What are group-level traits?}

Consider two armies squaring off. On one side we have the Barbarian Horde, a ragtag group of bloodthirsty killing machines. Each individual Barbarian is a deadly warrior, savage and merciless. Nevertheless, they have minimal group-level organization beyond attempting to avoid hurting one another - they are essentially an undifferentiated mass. On the other side is the Roman Legion. Individually, each Roman soldier is a skilled fighter, but would tend to lose in one-on-one combat with a Barbarian. The Romans, however, are a highly regimented unit. They have differentiated roles and hierarchical organization. Each soldier understands his role and how it relates to his fellow soldiers. In a fight between armies of equal size, the Romans will tend to dominate over the Barbarians, not because each Roman is better than each Barbarian, but because group-level organization allows the Romans to outmaneuver their opponents. Here I am using the terms "Roman" and "Barbarian" rhetorically rather than historically, but a dramatic historical example of this kind of confrontation is the famous Battle of Thermopylae in 480 BCE, in which a small but highly organized Greek force of about seven thousand men used a phalanx formation to successfully defend their front against Persian forces of more than 100,000 soldiers for a full week (before finally succumbing). The properties that allowed one group to triumph or persist against another in these cases did not belong to each individual group member, but rather emerged from the organized interactions between those individuals. I term such properties group-level traits.

Group-level traits are possible when individuals display both differentiation and organization. By differentiation, I mean that individuals take on different roles. The assignment of these roles may be based on differences in innate physical characteristics or abilities, in age or extent of experience, or in deliberate training and specialization. By organization, I mean that differentiated individuals coordinate and collaborate for a shared purpose. Grouplevel traits rely on organization, but the organization itself is not the trait. Group-level traits are related, but not equivalent to institutions, which are "the laws, informal rules, and conventions that give durable structure to social interactions in a population" (Bowles 2004, p. 47). Instead, a group-level trait is the phenotypic effect of social organization. Thus, examples of group-level traits are the music rather than the rock band, the election of a leader who reflects the public interest rather than the democratic voting system, the sailing ship's voyage rather than the crew positions, the economic surplus rather than the market economy.

The significance of differentiation and organization is well known in evolutionary theory in the context of the major transition from single-celled organisms to multicellular life (Maynard Smith \& Szathmary 1995; Michod 2007; Michod \& Nedelcu 2003; Michod \& Roze 1997; Okasha 2005). Indeed, there are a number of similarities in the distinction between individual cells and a whole organism and that between individual humans and emergent group organization. The somatic cells of a multicellular organism give up their right to reproduce without bound for the sake of propagating the germ cells that share their genomic sequence. Natural selection can act on individuals because the traits of the individual cells are subsumed into the larger organism; somatic cells cooperate with one another and inhibit the unbounded reproduction common to single-celled creatures (Michod \& Roze 2001). Although they each share the same genetic code, the cells are differentiated by experience, chemical environment, and gene expression.

Somatic cells meet the criteria for Darwinian evolution there is heritable variation that influences fitness (Jablonka \& Lamb 2005) - yet we do not usually think of the cells in the body as undergoing individual evolution (except perhaps in the case of cancer cells). Reproductive success for a multicellular organism translates not to the propagation of the traits of any one cell, but rather to the traits that emerge from the interconnection and cooperation among cells. A better-organized collection of cells is a fitter multicellular individual, and it is this organization that is selected for. Selection therefore operates on traits that involve the interactions of many individual cells. These traits do not exist within any individual cell, but only in their organized aggregation. It follows that if a heritable trait found in a group of multicellular individuals exists not as a property of each individual but as an emergent property of their organized interconnectedness, and if groups possessing this property increase their ability to propagate over groups without it, then selection will favor such groups.

Human groups organize in ways that produce emergent group-level traits. These traits produce between-group differences in genetic and cultural fitness and are heritable through cultural transmission. Group-level traits are not expressed by any single individual in the group, but emerge only from the structured organization of differentiated individuals. The reduction of culture and cultural practices to individual-level variants therefore misses an 
essential component of what enables human groups to succeed, as well as a key component in the evolution of cultures and societies.

\subsection{What is a group?}

To paraphrase Wilson (2002), a discussion of the role of group-level traits in evolution requires an unambiguous definition of groups. Yet groups may constitute quite different things at different times. The Roman Legion acts as a unit during battle, and the fate of each individual depends on the behavior and coordination of his brothers-in-arms. So the entire Legion is a group producing certain traits. Historically, however, soldiers in Roman battalions were drawn from many different cultural and ethnic populations, with diverse diets, languages, and customs. Off the battlefield, individuals organized into different group configurations, producing different types of traits. A deer hunter and a bowyer (bowmaker) might collaborate far from the fighting zone to their mutual benefit. Such a relationship, if largely uncomplicated by the actions of other individuals, would also constitute a group. Discussing the more traditional approach to multilevel selection theory, which deals with the selection of individual-level traits in a group context, Wilson writes: "When the trait is a social behavior, the fitness of an individual is determined by its own traits and the traits of the individuals with whom it interacts. These individuals constitute the group, which must be identified accurately to calculate the fitnesses that determine the outcome of evolution. It follows that groups must be defined separately for each and every trait." (Wilson 2002, p. 15). Group-level trait groups should be defined similarly. The trait-group, to use Wilson's (1975) term, is the collection of individuals interacting to produce the trait in question. A group may consist of a simple dyad or a population of thousands.

\subsection{Not all collective behaviors are group-level traits}

Collections of individuals can exhibit many behaviors that lone individuals cannot, but not all of these behaviors should be considered emergent group-level traits. For example, prey animals in large groups can use simple, individual-level rules to generate flocking, schooling, or herding behavior to more effectively find food or evade predators (Sumpter 2006). Collections of humans making independent evaluations can often make optimal decisions by averaging across all individual assessments (Surowiecki 2004). These kinds of collective behaviors are clearly important in the ecological behavior of humans and other species, and exert influence on evolution by creating new selection pressures (Lewontin 2000; Odling-Smee et al. 2003), yet are qualitatively different from group-level traits. In contrast, Woolley and colleagues (2010) recently presented a series of experiments showing that the collective ability of a small group to solve problems (the group's collective intelligence) emerged from communication between the group members and was uncorrelated to the intelligence of the individuals in the group. Though the authors did not investigate exactly how the groups solved problems, other researchers have shown that groups in which individuals possess different abilities are often better able to solve collective problems (S.E. Page 2007; Post et al. 2009).
Wimsatt (1997; 2006) discusses properties of systems that are aggregate, rather than emergent, as having four qualities: (1) the system property is invariant to rearrangement of the parts, (2) the system is qualitatively similar under addition or subtraction of parts, (3) the system property is invariant to regrouping of the parts in system subgroups, and (4) there are no cooperative or inhibitory interactions among the parts of the system that affect this property. Many collective behaviors are largely aggregate, exhibiting qualities 1-3. They result from a number of interchangeable individuals acting independently (though they each react to different local stimuli) and tend to have qualitatively similar behavior for a wide range of group sizes. Flocking birds, for example, may inhibit or elicit cooperative behaviors in one another, but birds could be added or removed from the flock without qualitatively altering the collective behavior. Meanwhile, grouplevel traits are emergent to a much larger degree, because they depend strongly on specific arrangements of differentiated actors in specific organizational roles (and do not meet aggregate qualities 1-3). The difference between aggregate and emergent properties is often relative, but a useful heuristic for distinguishing group-level traits from collective behaviors is that the latter depend strongly on the specific organization of differentiated individuals, whereas the former do not.

\section{Group-level traits and cultural group selection}

Darwin (1871) originally proposed that, at least in the case of humans, many psychological traits related to empathy and altruism would have been selected against at the individual level and so must have evolved because groups of cooperative individuals would outperform selfish groups. WynneEdwards (1962) later developed a theory of group selection in which he proposed that predators should exhibit restraint in reproduction to avoid overexploiting their resources and that such restraint could evolve as a result of being groupbeneficial. However, the logic of Wynne-Edwards' argument was rightly criticized (Williams 1966) as being incompatible with natural selection - less "prudent" predators would outcompete their more restrained neighbors.

If well-defined groups compete, however, and the variance of a trait tends to be higher between than within groups, then it is theoretically possible for an altruistic, group-beneficial trait to emerge, because groups with many individuals possessing such a trait will have higher mean fitness than groups with fewer altruists. This point is well supported by formal models (Henrich 2004a; Price 1972; Wilson 1975; Wilson \& Dugatkin 1997) and has found empirical validation (Goodnight \& Stevens 1997; Wade 1978). This newer group selection perspective, closer to Darwin's original ideas than to those of WynneEdwards, has since been adopted as the multilevel selection (MLS) framework (Bijma \& Aanen 2010; Wade et al. 2010; Wilson 1997; Wilson \& Sober 1994; Wilson \& Wilson 2007).

Recently the MLS perspective has been applied to culturally transmitted traits in humans (Boyd \& Richerson 1985; 2005; Henrich 2004a; Richerson \& Boyd 1998; Soltis et al. 1995; van den Bergh \& Gowdy 2009) under the heading cultural group selection. This perspective applies the same logic as MLS (and shall hereafter be referred to as cMLS) and works as follows. Altruists, who contribute to the 
average group fitness at a personal cost, are outperformed by selfish individuals within groups, but altruistic groups outperform selfish groups. Therefore, if the variance within groups is sufficiently low and the variance between groups is sufficiently high, between-group selection can have a bigger influence than within-group selection, and selection for altruistic traits occur. The primary argument that has been pitched against MLS is that these conditions of low within-group variance and high between-group variance are rarely met in most naturally occurring species. Proponents of cMLS have presented strong evidence that this argument does not apply in the case of culturally transmitted traits, because human psychology and cultural norms tend to maintain between-group differences (Boehm 1997; Boyd \& Richerson 2005; Chudek \& Henrich 2011; Henrich 2004a; Laland et al. 2000).

In discussing the adoption of the cMLS perspective, Laland et al. (2000) have written: "Group selection of cultural rather than genetic variation requires a 'frame shift' of replicator, because it is not genes that are selected for, but rather groups of individuals expressing a particular cultural idea" (p. 143). Nevertheless, the traits in question in the cMLS perspective - whether "cultural ideas" or the more behavioral "cultural variants" (Richerson \& Boyd 2005) exist at the level of individuals. Although fitness may be evaluated at the group level by averaging across group members, each trait in question is still the property of individuals in that group. I argue that the frame shift proposed by Laland et al. (2000) is not incorrect, but it is incomplete. The cMLS view does not at present account for the fact that collaborative behaviors, requiring differentiated and structurally organized roles, have played an essential role in the success of human groups. Group-level traits exist fundamentally at the level of groups and can therefore only be defined in those terms. A group may be partially successful because its members express a particular cultural idea. But a large part of group success comes not from an aggregate of identical individuals each expressing a unitary idea, but from the organization of a well-defined collection of differentiated individuals all participating in a group-level behavior, as in the case of the Roman Legion.

A related view has been advocated by MLS theorists seeking to distinguish between effects of group structure on the fitness of either (1) the constituent individuals or (2) the groups themselves, with the former view designated MLS1 and the latter MLS2 (Damuth \& Heisler 1988; Michod 2005; Okasha 2005; 2006). On the surface, MLS2 may appear to be the appropriate framework for discussing the evolution of human group-level traits. Indeed, some presentations explicitly discuss emergent traits as properties of the collective group, rather than belonging to the individual group constituents. However, these "emergent" traits are, in general, aggregate properties of the collective as a whole, rather than produced by interactions among its members. Two examples given by Okasha (2006) are (a) the geographical range of a species of mollusk and (b) the degree of morphological differentiation between castes in a colony of social insects. These traits are to some degree emergent in the sense discussed by Wimsatt (1997; 2006), yet both exhibit aggregate quality 1 , in that they are invariant to a rearrangement of parts. Example (a) does not depend on either differentiation or organization-but only on the sum of the ranges of the individual species members. Example (b) describes differentiation and organization. However, the trait does not emerge from the collection of individuals possessing those properties, but is merely a statistical description of their organization. As such, the MLS1/MLS2 framework is not yet well developed enough to account for the evolution of group-level traits. Moreover, as discussed earlier, human trait-groups are not fixed collections but are defined by the traits themselves. Individuals may become involved in many groups, with varied memberships and purposes, throughout their lives. Thus, although patterns of organization and differentiation may propagate through culture and social learning-discussed in more detail in subsequent sections - a trait-group is not itself consistent enough over time to constitute a unit of selection as defined by MLS2.

Several authors have previously discussed group-level traits produced by organization and differentiation in the context of human cultural evolution (e.g., Boehm 1997; Caporael 2001; Henrich 2004a; 2010; Richerson \& Henrich 2012; Wilson \& Sober 1994). However, these authors have typically failed to acknowledge a special significance of such traits and have instead discussed them as equivalent to aggregates of individuals all exhibiting individual-level traits (i.e., not defined by organization and differentiation). For example, Wilson and Sober (1994), in their famous paper "Reintroducing Group Selection to the Human Behavioral Sciences," write: "It is also crucial for our hypothesis that group-level functional organization is, in some sense, superior to what can be accomplished by individuals when they are free to pursue their own selfinterest" (p. 604). Yet the idea of "group-level functional organization" is not explored further in their paper.

Some considerations of cultural group selection have gone beyond ignoring within-group differentiation and have actively argued for the importance of within-group homogeneity. Boehm (1997), for example, has made this suggestion in his discussions of "egalitarian behavioral syndrome" in small-scale societies, which involves moralistic norms to control antisocial behavior and thereby dampens within-group phenotypic variation and amplifies variation between groups. The argument focuses on the fact that egalitarian norms allow maximal sharing of resources, which in turn purportedly maximizes group success. However, this argument holds only as long as the baseline fitness, beyond the sharing or withholding of individual resources, is constant between groups. Any fitness gains (or losses) based on group organization will be missed by cMLS models that do not consider the influence of within-group differentiation and organization. In contrast, such withingroup differentiation allows for the emergence of grouplevel traits and can thereby permit structured groups to have an evolutionary advantage over similar groups with more homogeneity and less emergent structure. Although selection on both biological and cultural traits may favor homogeneity in some cases, such as in the emergence of early hunter-gatherer societies, the historical record emphatically supports the conclusion that structured differentiation is often beneficial to group success.

\section{Beyond cooperation}

The majority of the theoretical and empirical literature on social and cultural evolution, in humans as well as other 
species, has focused on cooperation. This stems in part from the seemingly mysterious questions of how cooperation can first evolve and how it can be maintained. After all, if I help you at a cost to myself and you do not reciprocate, then I suffer a cost and you reap all the benefit. Half a century of research on cooperation has largely solved this puzzle. Cooperation can evolve if individuals with heritable cooperative tendencies can positively assort (Fletcher \& Doebeli 2009; Smaldino \& Schank 2012a; Wilson \& Dugatkin 1997), meaning that they tend to interact more with one another than at random. Positive assortment can be facilitated by a variety of mechanisms, including kin recognition, cognitive bookkeeping (McElreath \& Boyd 2007), spatial assortment with limited dispersal (Koella 2000; Smaldino \& Schank 2012a), or goal-directed movement away from free riders (Aktipis 2004; Helbing \& Yu 2009; Smaldino \& Lubell 2011). Free riders will of course be a perennial problem, in part because the relative benefit of free riding increases with the frequency of cooperators. However, once established, cooperation can be enforced by social institutions such as direct reciprocity (Trivers 1971), indirect reciprocity (Nowak \& Sigmund 2005), reputational exclusion (Smaldino \& Lubell 2014), and punishment (Boyd \& Richerson 1992; Henrich \& Boyd 2001).

So, although there are still open questions related to the evolution and maintenance of cooperation, its presence is no longer a mystery. Its ubiquity in human society, far beyond that found in other species, presents perhaps more of a puzzle. Humans live in large groups of unrelated individuals and are inherently group-minded. The question often posed is: How is it that we cooperate so much? Proponents of cultural group selection have argued forcefully for a two-step process (Chudek \& Henrich 2011; Richerson \& Boyd 2005). First, cultural learning biases (e.g., conformity, learning from prestigious individuals) and a suite of psychological mechanisms evolved for dealing with social norms combine to maintain within-group similarity and between-group variation. Second, competition between groups selects for groups with cooperative social norms. Although the logic of this process is sound, it also downplays the important role of within-group variation in making groups of individuals working together so successful. For example, norms of organization and differentiation may not be fully carried in the minds of individuals, but may be transmitted through inheritance of social structure (Laland \& O'Brien 2012), as well as through the maintenance of variation within the population to produce individuals who take on differentiated roles.

\subsection{Cooperation vs. collaborative interdependence}

In the Russian arctic, Inuit hunters forage for seabird eggs during the summer months when seals and walrus are scarce (Fothergill \& Berlowitz 2011). These birds nest on sheer cliffs, making accessing their eggs a challenge. The hunters' solution is to fasten a strong rope to the waist of one individual and to have the other members of the team lower him down the cliff to collect eggs into a leather bag. Adjustments in the rope tension are made through verbal communication, and the climber is pulled up when his bag is full. Through this collaborative exercise, enough eggs can be collected to feed all the hunters and their families. In many respects, this activity is obviously a cooperative behavior. Each individual is contributing time and energy to a common good. Yet, the group-level behavior is defined not simply in terms of individuals donating or withholding contributions, but in terms of each individual doing his own part in a coordinated and organized manner. These hunters are doing more than cooperating: they are collaborating.

Much of the work on the evolution of cooperation is based on social dilemma games such as the prisoner's dilemma, public goods, snowdrift, and stag hunt games, and research tends to focus on how to evolve and sustain general practices of cooperation. An implicit assumption of the payoff structures of these cooperative games is that cooperative or altruistic acts are completely general; that is, interacting with one cooperator is just as good as the next. Some work has considered cooperation only between members of the same group (Antal et al. 2009; Axelrod et al. 2004; Hammond \& Axelrod 2006; Jansen \& Van Baalen 2006), but acts of cooperation within groups are still treated as equivalent. This structure misses a couple of important points about how cooperation works in human societies. The first is that cooperation is often domain specific. For example, some Indian villagers have been known to cooperate across castes in some domains (e.g., farming) but not others (e.g., marriage) (Waring 2012).

The second and much richer point is that with whom one cooperates matters. It matters not only to the individuals involved, but also to other members of the group. Generically, assume individuals A, B, and C are all members of a larger group, and further assume that they all tend to cooperate with members of their group. Whether A chooses to interact with $\mathrm{B}$ or with $\mathrm{C}$ matters, not only for A's individual payoff, but also to the quality of the public good available to A's group. In other words, some structural relationships are more productive, in absolute terms, than others. As an example, consider a hypothetical smallscale society of arctic hunters. A seal hunter and a kayak builder have a high incentive to cooperate with one another. They have each developed skills that require those provided by the counterpart. If the kayak builder gives the seal hunter a boat, he can be rewarded in turn with sustaining meat. The kayak builder has a very different relationship with a rival kayak builder, who may cooperate by sharing knowledge or building materials, but also competes for customers (and therefore the returns from hunting voyages). Moreover, the seal hunter should choose carefully with which kayak builder he cooperates. If he chooses one with less skill, he may have to expend more time and resources to guide his craft on the water and to repair it if it is more prone to damage. This will lead to a less fruitful hunt and to less food for the group overall. For a related discussion, see Calcott (2008).

Some work on game-theoretic models of cooperation may tacitly assume that differentiation and specialization are sources of the non-zero sum nature of scenarios such as the prisoner's dilemma. The omission of individuallevel diversity and differing payoffs for different constellations of collaborators is often a useful simplifying assumption. Nevertheless, an inclusion of that diversity will help to shed a brighter and broader light on the forces underlying human evolutionary success, not only for evolutionary researchers, but also for students learning to think about human cooperation through the abstractions 
of game-theoretic models. The real story of how large-scale cooperation became established during the evolution of behaviorally modern humans is likely to have involved a trajectory of organization and differentiation that cannot be captured by more traditional cooperation models. As humans evolved to live in groups, group behaviors such as cooperative breeding and collective foraging allowed them to adapt to environments too harsh to sustain noncooperative individualists (Bergmüller et al. 2007; Hill \& Hurtado 2009). Eventually, such adaptations may have created the necessity for interdependence, in which survival without some minimal amount of cooperation or aid from other group members was impossible (Smaldino et al. 2013a; 2013b). Interdependence sustains cooperation and provides a stable environment of mutual aid in which differentiation, division of labor, and complex group organization can emerge.

The simplest form of interdependent collaboration stems from a diversity of abilities when a group is engaged in a common endeavor. Diversity creates a broader base of ideas for solving problems and innovation. Organization and management scientists have shown that for a variety of problems, problem-solving teams whose members exhibit diversity in training background and styles of thinking are often more effective than teams consisting of experts in any one area (S. E. Page 2007; Post et al. 2009). Henrich (2004b) has also shown that greater diversity in observer inferences during social learning can promote adaptive cultural evolution for individual-level traits.

Over time, even subtle differences in abilities and circumstances can give rise to differentiated individuals who are dedicated in their roles and deeply specialized. Formal division of labor is required for many group-level behaviors. Division of labor allows specialized individuals to achieve depths of skill impossible for the generalist. A group with division of labor can easily outperform a group of generalists, but only if those specialists reinforce one another by providing what others lack.

In this light, cooperation becomes more nuanced and gives way to collaboration, in which specialized individuals form an interdependent network of skills, personalities, and experiences that all contribute to group success. This also suggests a reconsideration of the value some researchers have placed on egalitarian norms (Boehm 1997; Wilson \& Sober 1994). For example, Wilson and Sober (1994) discuss the Hutterite society, a communal branch of Anabaptists that originated in Central Europe in the sixteenth century. Hutterites place high value on egalitarianism, which minimizes within-group variation and should, in the cMLS framework, promote group success. But this view can also overlook the importance of differentiation and organization within groups, which are essential for generating group-level traits. Wilson and Sober remark that in present-day (i.e., 1994) Canada, "Hutterites thrive in marginal farming habitat ... and would almost certainly displace the non-Hutterite population in the absence of laws that restrict their expansion" (p. 605). Wilson and Sober (1994) are arguing not only that egalitarians norms have driven the success of the Hutterites, but also that it is lucky for Canada that its government has regulatory laws in place to stem the otherwise unstoppable tide of Hutterite expansion. In my view, a key element of this story is that the more "mainstream" society of Canada and its government possess organizations such as regulatory agencies and law enforcement that permit a seemingly effortless impediment to Hutterite expansion. Canadian culture in this sense outcompetes Hutterite culture not through increased norms of cooperation, but via properties that emerge from complex social organization. It is clear from models and from studies of small-scale societies that, in a population of near-equals, increased cooperation and egalitarianism can promote group success. When dealing with complex societies, however, a focus on cooperation is limited because it misses the influences of collaborative interdependence and group-level traits. This point is supported by a recent game-theoretic model demonstrating that economic stratification and division of labor within a structured society could generate higher total group payoffs than egalitarian norms (Henrich \& Boyd 2008). Importantly, such a payoff differential creates the conditions for selection between groups. Moreover, Henrich and Boyd's (2008) model provides a rare example of an explicit evolutionary model of cooperation among differentiated individuals. The authors assumed that all interactions were cooperative but posited different payoffs for different dyadic pairings. Further work on the evolutionary significance of interdependent collaboration will be illuminating.

The aim of this discussion is not to downplay the importance of cooperation in human evolution. Cooperation is essential in many contexts of human life, and the value of promoting cooperation and deterring free riding should not be understated. Nevertheless, cooperation only gets us part of the way to explaining the emergence and sustainability of complex group-level behaviors. Once cooperation between individuals evolves, the stage is set - via persistent association, interdependence, and cultural transmission for the evolution of nuanced collaboration between individuals with differentiated roles in a meaningful social organization.

\section{Explaining the emergence of group-level traits}

Group-level traits allow groups to do things that aggregates of individuals cannot do otherwise. Humans build skyscrapers, sail ships, and create complex tools, infrastructure, and modes of transportation. We pass down traditions of learning that enable cumulative improvements and innovations, including knowledge that no single generation of social learners could acquire on its own. How do complex group-level traits emerge from properties and behaviors of individuals?

The eusocial insects, which includes many species of ants, termites, and bees, also exhibit group-level traits with differentiation and organization. The construction and defense of ant bivouacs or giant termite mounds are neither the product of a single individual nor that of an aggregate of identical workers. Rather, it takes the interdependent collaboration of workers and soldiers, drones and queens. Eusocial insect colonies, however, are collections of individuals with a high degree of genetic relatedness, and the emergence of group-level traits in these species is encoded at the level of the genotypes of the foundress queens, their mates, and their offspring. ${ }^{1}$ The colony is in some sense an extended phenotype of the queen (Reeve \& Hölldobler 2007), with between-group differences 
explained by genetic (and also environmental) differences, and within-group differences explained by variation in environmental stimuli, triggering differential gene activation during development. In contrast, group-level traits in humans are transmitted culturally rather than genetically, requiring different explanations for their emergence and evolution. In this section, I focus on proximate mechanisms that contribute to the emergence of group-level traits.

Uniquely, humans live in large, complex societies full of cooperative and collaborative relationships between nonkin, and so the extended phenotype explanation does not apply to selection on human group organization. Humans seem built to learn from one another, and most differences between groups of humans appear to be largely the result of learning rather than genotype (Richerson \& Boyd 2005; Baldini et al., under review). This statement is well supported by the empirical literature on human development (Chudek et al. 2013; Harris 2012; Baldini et al., under review) and cultural psychology (Nisbett et al. 2001; Nisbett \& Miyamoto 2005). Even the motherinfant attachment relationship, which is for most humans their first participation in a group-level trait and is in many ways a human universal (Grossman \& Grossman 2006), is influenced by the culture and past experiences of the mother (van Ijzendoorn et al. 2006). Is it possible that group-level traits are fully explained by socially learned individual-level traits? In order to explain grouplevel traits, the emergence of differences among individuals within a single group, and the subsequent organization of those differentiated individuals, and their coordinated behavior must be accounted for. Bearing in mind the complications in the proximate/ultimate distinction ${ }^{2}$ (Laland et al. 2011), three possible proximate explanations for the emergence of group-level traits present themselves. Each is only partly explanatory on its own - a fuller picture requires the incorporation of all three.

\subsection{The role of leaders}

First, some individuals may possess a plan or leadership quality that allows them to direct others into some organization and guide them through a task. Indeed, a person can - and often does - carry around large portions of the roles, rules, interactions, and institutions for group-level traits in her mind. Not only that, but the individual can use that information to predict how a group will behave under such a structural organization. Indeed, much of modern social science has been dedicated to the conceptualization and description of the ways in which organizational changes will affect group outcomes. The crux, however, is that individuals cannot express those grouplevel traits. To do so, they need a collective of cooperative, differentiated individuals. They have to start a company, form a band, inspire a religion, lead a nation. This sort of behavior is at the heart of modern human history.

Moreover, it is often impossible to precisely guide other individuals to perform exactly as one wishes. An old adage in the film industry is that "good directing is 90 percent casting." In other words, getting the right group of people in the right roles is essential for achieving good results. This is not to suggest that individual leaders, such as directors, architects, and CEOs, are not important to the operation of group behaviors. Rather, their roles are limited by other forces, including not only the characteristics of the other individuals involved, but also the constraints related to environment, infrastructure, and culture. Furthermore, leadership does not explain the existence of suitable differentiated individuals to perform a given task.

Maynard Smith and Szathmary (1995) contrast the function and complexity of a termite mound with that of a human building, noting that although the two resemble one another in many ways, the termite mound "differs in that not one of its builders had a picture of the completed structure before building started" (p. 257). Although many of the people involved in manufacturing human constructions have a mental picture of the finished object, it also true that, in many cases, no single person has either the knowledge or the ability to build that object. This was noted by the economist Leonard Read (1958) in his whimsical essay "I, Pencil" in which he suggests that no single person can make something so seemingly simple as a pencil. Indeed, much of the technology in the developed world is so complex and built on cumulative technologies, designs, and materials that thousands if not millions of individuals may be involved in their construction, none of whom know how to make the completed product (and few of whom may ever use or even see the completed product!) (Ridley 2010). ${ }^{3}$ Therefore, although important, planning and leadership cannot fully explain group-level traits.

\subsection{The emergence of division of labor}

Second, although individuals may all be exposed to the same socially transmitted information via common cultural environments, environmental and biological differences may create the opportunities for differentiation. Differentiation and division of labor may emerge when small differences are exacerbated through the exploitation of opportunities. R. E. Page and colleagues (Fewell \& Page 1999; Page \& Mitchell 1991) have developed a model of division of labor in insect societies in which differentiation emerges when intrinsic variation exists in the ability to perform a behavior and performing that behavior reduces the need for others to complete the specific task. It seems reasonable to propose that some differentiation in human societies may also be explained in this way. For example, most competitive Canadian hockey players are born in the first few months of the year (January, February, March). This is not because of astrological providence, but rather because January 1 is the age cutoff for most youth hockey leagues. In these leagues, players born earlier will tend to be bigger and stronger than their co-players and will tend to perform best and, subsequently, will have more opportunities and more coaching, widening the performance gap (Gladwell 2008). Since not everyone can play in the pro leagues, some players will get dropped, and on average, rosters will include more players born early in the year. The widening of intrinsic differences through education and experience therefore creates opportunities for differentiation. Here, selection can operate at the individual level, with a frequency dependency for various phenotypes depending on the priority of the organizational roles to which those phenotypes are best suited. However, two questions illustrate that this is not a complete explanation of group-level traits. First, how do opportunities for improvement in a given role arise? That is, how 
are individuals selected for specific roles, and how do they get their role-specific training? Second, and more importantly, where do the available roles come from in the first place? A hockey player can only play hockey if opportunities to do so are available and can only turn professional if pro hockey leagues exist. Similarly, a soldier must be trained in an active military establishment or by cultural traditions of warriorhood, and a novice canoe builder must generally be taught by an elder canoe builder.

\subsection{Repeated assembly}

A third explanation for group-level traits incorporates the facts that humans have cumulative culture, a long developmental trajectory, and lots of generational overlap. Grouplevel traits may be examples of what Caporael (2003) has termed repeated assemblies. These are "recurrent entityenvironment relations composed of hierarchically organized heterogeneous components having different temporal frequencies and scales of replication." (Caporael 2003, p. 77) The repeated assembly view as applied to individual development stands in counterpoint to the view that contributions from genotype ("nature") and environment ("nurture") may be viewed additively or, at best, as interacting in fairly simple ways; this latter view characterizes some work in evolutionary psychology (e.g., Tooby \& Cosmides 1992) and behavioral genetics (Bouchard \& Loehlin 2001; Kendler \& Greenspan 2006). Instead, development is characterized by a process by which the individual is assembled in overlapping cycles - aspects of the current cycle seed the beginning of the next - with cycles occurring at varying timescales and involving varied interactions between the individual, the external environment, and other social interactants. Stages of development may be scaffolded by more experienced individuals, as well as environmental and cultural structures that promote learning and transitions to subsequent stages (Wimsatt \& Griesemer 2007). The development of organized groups, exhibiting emergent group-level traits, may occur in a similar manner. A major difference is that the components of the group may change over time, while the group's structure may change little. The inverse may also occur: group structure may change even though the constituents do not, or the constituents may change only in the sense that the same individuals change roles to fit a new structural organization. The evolution of such organizational structure is therefore qualitatively different from selection on individual-level traits.

Consider also the importance of pedagogy and the development of social roles. In any given social context, individuals may have roles related to the structure of the organization and the needs of the related endeavor. These roles may be entrenched in tradition and infrastructure, but they may also be in near-constant flux. Adult humans do not spring into existence fully formed with a set of relevant skills. Throughout their development, individuals are drawn to different roles through opportunity, experience, and epigenetically developed predispositions (Gottesman \& Hanson 2006; Nijhout 2003; Simonton 1999). As novices in an organization, they learn by instruction, imitation, and exposure from more experienced individuals. Once they themselves become experienced, they may take on the role of mentor to more junior members of the organization, and there is no reason this scaffolding cannot be multitiered.
Human cognition is highly adapted for social coordination, to a much greater extent than our nearest primate relatives (Herrmann et al. 2007). Although chimpanzees may engage in collective behaviors, such as those for hunting or defense, Tomasello and colleagues (2005) have argued that "in these interactions each individual does basically the same thing, they just do it in concert" (p. 685). In contrast, humans collaborate in organized groups of differentiated individuals, and much of our evolved psychology is related to the fact that we live in social groups - perception, cognition, and behavior relating to social relationships are paramount (Brewer 2004; Caporael \& Brewer 1995).

Humans have species-specific cognitive and perceptual mechanisms that allow for scaffolded social learning, which likely facilitated the emergence of cumulative culture (Csibra \& Gergely 2011; Moll \& Tomasello 2007; Tomasello et al. 2005). These psychological mechanisms likely coevolved through mutual reinforcement with social structures that promoted coordinated communication and organization (Gong \& Shuai 2012), allowing generations of early hominins to develop organizational structures of increasing complexity over many generations. In general, modern humans have deep-rooted dispositions toward cooperation and collaboration, far beyond those of other species (Bowles \& Gintis 2011; Nowak 2011; Richerson \& Boyd 2005; Tomasello 2009). Once these collaborative instincts are present, different distributed collective adaptations - group-level traits - can be transmitted among groups, because people remain largely unspecialized in morphology but can become extremely behaviorally specialized during development.

\section{The maintenance and evolution of group-level traits}

People do not simply assess and imitate individual behaviors. They adopt entire suites of behaviors, perceptual norms, and decision heuristics (e.g., "What would Jesus do?"). Norm-enforcing institutions allow suites of cultural traits to be transmitted wholesale, which in turn allows for something analogous to natural selection to operate on the group-level traits that emerge from cohesive collections of individual-level traits. Mechanisms that stabilize group-level cultural identities have been well documented. For example, initially arbitrary ethnic markers can evolve and stabilize because they help facilitate within-group interactions, which tend to be more important than extragroup interactions (McElreath et al. 2003). Durham (1991) discusses a number of ecological, psychological, linguistic, and cultural barriers to the blending of cultures, which he terms transmission isolating mechanisms (TRIMs). These mechanisms ensure that cultural identities remain relatively stable, even when individuals from different cultures interact. TRIMs are the major reason that cultural evolution at the level of groups, in the cMLS sense, can occur. TRIMs also help to stabilize the differentiated roles needed for the successful functioning of an organized group-level trait. As a simple example, positions within a formal organization may only be filled when there is a vacancy, and there are incentives to perform one's stated role and not impinge on another's responsibilities.

Mechanisms also exist that allow within-group differences and patterns of organization to be maintained and 
transmitted. For example, narrative stories and other media are important factors in the preservation of cultures and customs. An important but rarely made point about the role of narrative in human evolution is that a cultural tradition of story and mythology gives the members of a culture common referents - a perceptual lens through which to assess and communicate a particular situation. This claim is supported by evidence that culture and social learning can have important influences on basic cognitive and perceptual functioning.

L. F. Barrett and her colleagues have proposed that the common emotion categories (e.g., fear, anger, happiness) are not true natural kinds, but rather emerge through attractor states that arise as neurobiology is shaped by language and culture (L. F. Barrett 2006; L. F. Barrett et al. 2007; Lindquist et al. 2012). In other words, different neurophysiological states may lead to similar arousal levels and may be cognitively and phenomenologically mapped as a particular emotion. This process of fast categorization may aid in the adaptive rapid assessment of a situation and may interact with fast and frugal decision-making heuristics (Cunningham et al. 2007; Gigerenzer et al. 1999; Smaldino \& Schank 2012b). Analogously, there are myriad ways in which humans can perceive any given set of circumstances. Perception is constrained in part by our biology, but culture also constrains even our basic perceptions of a situation (Nisbett \& Miyamoto 2005; Smaldino \& Richerson 2012). For example, Masuda and Nisbett (2001) showed American and Japanese university students animated underwater scenes with a focal fish. In a recall task, Americans were much better identifying fish they had seen independent of background information, but Japanese students were much better at remembering details of the background scenes. Cultural differences in patterns of perception and memory fit larger cultural differences in epistemology and styles of thinking that exist between East and West (Nisbett et al. 2001). Nisbett and Miyamoto (2005) have even proposed that cultures tend to shape their own environmental landscapes to reinforce cultural perceptive norms.

If culture can influence how members of that culture perceive situations, then culturally transmitted norm-enforcing institutions should indicate norms of organization and roles within those organizations. Narratives, mythologies, and other media take on the important role not only of maintaining differences between cultural groups, but also of maintaining differences between individuals within groups and organizational structures for those individuals. Institutions that transmit these patterns of organization are in some (very loose) sense the vehicles through which group-level traits are transmitted.

\subsection{Religion as a norm-enforcing institution}

Dennett (2006b) has proposed that "the key to our domination of the planet is culture, and the key to culture is religion." Although I believe that religion is only one among a number of important norm-enforcing institutions, it has also historically been a particularly effective mechanism for transmitting whole suites of cultural norms. Religion may be viewed as one of the primary methods by which a culture maintains its norms of social organization and by which it propagates those norms (Atran \& Henrich 2010; Wilson 2002). In a now-classic study, Cavalli-Sforza and colleagues (1982) found that religious traits are among those most strongly influenced by vertical transmission, especially between mothers and offspring. Religious identity is therefore both highly susceptible to cultural transmission and unlikely to change through adult peer influence. This may partly be because religions are highly entrenched in cultures as a force for the differentiation and organization of individuals and are thus more difficult to change compared with institutions that only affect individual-level traits. However, large-scale religious conversion is not unknown. In this way, a suite of individuallevel traits and organizational norms can be transmitted wholesale from one cultural group to another.

There is a particularly enlightening historical example of a suite of cultural norms being mis-transmitted from one group to another, because the two cultures lacked sufficient common ground (Clark \& Brennan 1991) to effectively understand the context of the other's organizational roles. These are the famous cargo cults of the Pacific Islands, and they provide an example of something analogous to mutation in the transmission of group-level traits (Dennett 2006a). A number of times in the last few hundred years, Europeans and Americans landed and set up shop on certain Pacific Islands, which were home to small-scale societies with traditional practices of ancestor worship. Some of the (often arbitrary) organizational traits of the Westerns were perceived by the islanders as practices through which their gods must have given them "cargo," a blanket term denoting the Westerners' wondrous material wealth. During World War II, Americans set up a base on the island of Efate and recruited workers from among the islanders of nearby Tana. Soon the people of Tana, having heard tales of the colossal wealth and technology of the Americans, began marching in parades, marking "USA" on their chests, and carving bamboo figurines of American warplanes, helmets, and rifles for use as religious icons. During festivals, elders would perform a dance based on American military drills. This was ostensibly in hopes that whatever gods bestowed cargo on the Americans would bless them similarly. What is especially fascinating in these examples is that not only were individual behaviors transmitted, but so were complex group-level traits and patterns of role differentiation. Islanders built plane runways, with some individuals stationed as flaggers and others up in towers wearing headphones made from coconuts (White 1965). The cargo cultists appear to have gotten the idea that, in order to receive cargo, they needed to do more than adopt particular behaviors - they needed to adopt particular social structures. This is significant, because social transmission of behavior is typically assumed to occur at the level of individuals. Here instead we see the transmission of social organization.

\subsection{The adaptiveness of group-level traits}

Modern Christian religions provide an example of how group-level traits might propagate more reliably. Religious organizations often include the office of missionary, in which church members are rewarded for bringing new individuals or, even better, new communities into the fold. The Church of Latter-Day Saints (LDS, Mormon) has gone so far as to make missionary work a developmental stage in the lives of all its members, which may partially account for why LDS is one of the fastest growing religions 
in many parts of the world (Grammich et al. 2012). As another example, consider Catholicism, which has been so important to the success of certain cultural groups (and remains so in some parts of the world). There, functional differentiation of roles has given rise to the priesthood, a set of individuals whose genetic fitness is zero but who exert a major influence on the cultural transmission of the norms and group-level traits associated with the Church. Direct proselytizing is only one way, however, by which group-level traits may spread.

Norms that facilitate more effective group organization will spread because the groups that exhibit the associated group-level traits will outperform groups with less effective organization. The Roman Legion will defeat the Barbarian Horde nine times out of ten. ${ }^{4}$ This principle of organization triumphing over sheer numbers is itself a common trope in Western cultural narratives. Consider, for example, the number of films in which a ragtag group of scrappers beat some sort of corporate behemoth by cunning and coordination. In this way, a focus on group-level traits may become more likely to guide organizational decisions.

Some group-level traits may promote not only themselves, but also the persistence of other cultural traits (including other group-level traits), in a manner similar to genetic linkage. For example, democratic norms, by which the citizenry vote on policy choices, can maintain many normative aspects of a culture because ideas that represent large deviations from the status quo are unlikely to receive a majority of votes (Nagel 2010). On a smaller scale, consider a musical group. A rock band is not a collection of individuals all with a "musician trait." Rather, the individuals play different instruments and often have quite different skills and qualities. The collectively formed musical group exhibits group-level traits that not only propagate themselves (in the spread of the music and the formation of future bands), but also ideas and norms associated with both the music and personalities of the individual musicians, band identity, and related subcultures. Returning to the subject of religion, Atran and Norenzayan (2004) have persuasively argued that religious rituals, which tend to involve specialized roles (Barrett 2000), often serve an important function in maintaining group cohesiveness and commitment to the group's welfare among its constituents.

\subsection{Technology and the adaptive response of group-level traits}

Groups of humans can differentiate and organize based on learned traditions and the interplay between biological predispositions and cultural opportunities. However, many group-level traits also rely on characteristics of the environment, including the social environment, which can irrevocably change from one generation to the next. Perhaps the simplest characteristic of the social environment is population size. Isolated from mainland Australia after the seas began to rise after the last glacial period, humans on Tasmania were stranded on an island that could not sustain more than a few thousand people. Over several thousands of years, the Tasmanians lost previously held technologies (e.g., bone tools) and never evolved others (e.g., coldweather clothing, fishing hooks) that readily developed in Aboriginal communities on the Australian mainland (Davidson \& Roberts 2009; Diamond 1978; 1999;
Henrich 2004b). Isolated from the mainland, they had no influx of new technologies and too small a population to successfully sustain their existing complex technologies. Recent theoretical work has suggested that if complex technologies are more difficult to learn and individuals vary in their ability to imitate the best among them, then complex skills can fade away in small populations (Henrich 2004b; Powell et al. 2009; but see also Read 2006; Vaesen 2012). This pattern of technology loss as a result of a shrink in population size has also been observed in several other populations (Boyd et al. 2011), and a recent analysis of indigenous marine foraging societies of Oceania found that both population size and contact with other populations predicted the technological complexity of their toolkits (Kline \& Boyd 2010). The evolution of complex group-level traits may therefore require some threshold population size in order to generate sufficient numbers of organized and specialized individuals. As such, technological complexity can be properly considered a group-level trait.

How are technologies maintained? Clearly, cumulative social learning and innovation across generations are necessary to build many things that a single human being could not invent from scratch. Beyond this, however, social networks, division of labor, and cultural traditions also contribute to the maintenance of innovation. Recall the earlier discussion of the complexity of the pencil. Certain types of organizational structures might be necessary to maintain even simple technologies. A sufficiently large population is, in turn, necessary to maintain the levels of complexity in differentiated social networks required to sustain complex technologies. Because complex technology often requires significant division of labor, the costs of specialization may be outweighed by the gains only when the specialist has enough customers and collaborators to be meaningfully useful. Thus, as the population of Tasmanians fell (and lost contact with outsiders), the group-level traits necessary to sustain innovations were no longer possible. ${ }^{5}$ This makes further sense if we consider an inverse situation: the vastly complex technologies of the modern developed world. How many hundreds of millions of interconnected individuals are necessary to sustain the Internet? Smartphones? Airline travel? Innovations are abundant and sustainable precisely because our population is so large and intertwined.

\section{Moving between the levels of selection}

Human societies are high in what Wimsatt (1974) has called interactional complexity. Roughly, this means that an investigator wishing to make useful predictions must simultaneously consider the system from multiple descriptive perspectives, because elements of those different perspectives interact in a causal manner. We can describe societies at the level of individuals, in terms of nuclear families, kin groups, subcultures, and social classes. We can also include infrastructure and transportation, livestock and farming, religious rituals and linguistic traditions, and all this is on top of the descriptive complexity of an individual human. A complex society cannot be adequately described in terms of any single descriptive decomposition. In order to better characterize the behavior and evolution of human societies, we must move beyond a simplistic multilevel 
viewpoint that portrays groups as aggregates of relatively undifferentiated individuals and thus implies that it is coherent to speak of the "group level" and "individual level" separately. Groups are complex organizations of differentiated individuals, and individual behavior is constrained by group organization.

Some perspectives on social and cultural evolution have recently emerged which accept the idea that organization matters in evolutionary models. Certainly, plenty of work on spatial organization and mobility within and between groups has shown that these factors influence evolutionary dynamics (Durrett \& Levin 1994; Lion \& van Baalen 2008; Perc \& Szolnoki 2010; Smaldino \& Schank 2012a). Work on niche construction (Laland \& O'Brien 2012; Laland \& Sterelny 2006; Odling-Smee et al. 2003) has formalized and extended Lewontin's (1982) observation that individuals actively alter their environments, creating new selection pressures that can be inherited by subsequent generations. Even more recently, discussion has turned to an emphasis on developmental scaffolds as a force in cultural evolution (Caporael et al. 2014; Wimsatt \& Griesemer 2007), though this perspective has not been extensively modeled. Still, a between-levels perspective in which groups and individuals are separate but intrinsically connected and in which the organization of differentiated individuals creates new group-level traits has yet to be addressed.

\subsection{Group-level traits, MLS, and inclusive fitness}

Traditional formulations of MLS (MLS1) treat group effects in terms of positive assortment-in-group members assort, so the fitness of an individual is influenced by the individual fitness differential of its own traits plus the fitness differential provided by the social traits of its group members. Thus, an altruistic trait that extracts a cost from an individual but benefits group members can evolve if the degree of in-group assortment is high enough. As many have pointed out, this formulation is mathematically equivalent to an inclusive fitness approach that accounts for the degree of assortment without explicitly accounting for group structure (Bijma \& Aanen 2010; Bourke 2011; Marshall 2011; Wild et al. 2009; Wilson \& Dugatkin 1997). Nevertheless, many proponents of cMLS theory have argued that studies of human cultural evolution and gene-culture coevolution are best served by a perspective that accounts for group structure (Chudek \& Henrich 2011; Henrich 2004a; Laland et al. 2000; Richerson \& Boyd 1998; 2005). The arguments presented in this target article support this position and take it a step further. When it comes to human cultural evolution, the equivalency between MLS and inclusive fitness theory dissolves once a between-levels perspective incorporating group-level traits is adopted.

When group-level traits can emerge and be maintained through processes that do not require genetic relatedness, an individual's social environment cannot be reduced to the frequencies of various social phenotypes. Rather, the social environment includes the structured groups (exhibiting group-level traits) in which the individual participates, as well as those groups that influence the fitness and behavior of both the individual and her groups. Individuals partner with other differentiated individuals in an organized fashion, constrained by institutional and developmental processes. This organizational complexity means that accounting for the degree of assortment between individual trait types is insufficient to calculate fitness. It follows that a multilevel selection theory of cultural evolution that incorporates emergent group-level traits is not equivalent to an inclusive fitness approach that focuses only on individuals and aggregate conceptualizations of fitness in groups.

\section{Future directions}

The idea that the structured organization of differentiated individuals influences the behavior of human groups is not new. Cultural anthropologists, for example, have long recognized the importance of characterizing multiple levels of organization in their descriptions of cultures (e.g., Hinde 1976b). The fields of management science and organizational psychology are almost entirely devoted to studying aspects of the organization and dynamics of groups. In discussions of cultural evolution, however, group organization is often ignored. By ignoring the role of group-level traits in cultural evolution, as well as the evolution of those traits, researchers have overlooked a major force in the ecology and evolution of human societies. This is particularly true when attempting to extend cultural evolution theory beyond small-scale societies and into the more developed civilizations of the past 5,000 years. If complex organization and differentiation of roles were key factors in the cultural and genetic success of those peoples, then those factors should be examined head on.

A pressing problem concerns the understanding of how group-level traits are transmitted and how the relevant patterns of organization adapt and change. Other important problems involve the identification of cooperative and collaborative organizations that have culturally evolved, understanding how those organizations are related to each other, and incorporating in this framework how individuals can participate in multiple group organizations at the same time. Studying group-level traits in ecological and evolutionary contexts may require new methodologies or, minimally, the alteration of existing methods. A detailed outline of such methods is beyond the scope of this target article, but several possibilities readily present themselves. A general challenge for mathematical biology is the search for better ways to model multilevel systems (Cohen 2004). A focus on the emergence and evolution of group-level traits provides a suite of interesting problems for mathematical scientists. For example, models are needed that capture the difference between the social spreading of a particular individual-level trait and the emergence of group-level behaviors that rely on differentiation and organization. There is also a need for methods for identifying the presence of group-level traits and their evolutionary and developmental trajectories, as well as their effects on the evolution of individual genes and cultural variants (and vice versa). Theorists should work to develop models for how groups form, evolve, and coevolve with individual-level traits. Another important avenue of research concerns the influence of cultural niche construction (e.g., Ihara \& Feldman 2004; Laland et al. 2001), in which changes made by humans to their environments create new evolutionary pressures. The acknowledgment of group-level traits also has obvious importance for the 
Commentary/Smaldino: The cultural evolution of emergent group-level traits

evolution of institutions. The processes of institutional change and evolution have been subjects of interest for economic and political theorists for some time (Aoki 2007; Greif \& Laitin 2004; North 1990; Young 1998) and have more recently begun to be addressed from the perspective of cultural evolution theory (Bowles et al. 2003; Boyd \& Richerson 2008; Richerson \& Henrich 2012; van den Bergh \& Gowdy 2009). In all these cases, however, the significance of group-level traits has been ignored or downplayed. The incorporation of group-level traits into future models of cultural evolution is crucial.

\section{Conclusion}

Human groups can manifest structural features and behaviors that are more complex, highly coordinated, fluid, and diverse than groups of any other species. Although the information for group organization can exist in the minds of individuals, group-level traits cannot be expressed by individuals but only through the coordinated organization of group members. Group-level traits represent an important factor in human ecology and cultural evolution and are categorically different from aggregates of individual-level traits. Traits at the level of individuals are the bedrock of human behavior and encompass the phenomenological experience of being human. But organization matters. Emergent group-level traits allow one group to outperform another, and they alter the physical and social environment, providing additional selection pressures and opportunities for new behaviors. The history of modern humans is to a large extent the history of organization. Researchers interested in the evolution of culture, and in the coevolution of culture and genes, must consider the mechanisms by which group-level traits emerge and evolve.

\section{ACKNOWLEDGMENTS}

I am indebted to Josh Epstein, Jim Griesemer, Richard McElreath, Emily Newton, Pete Richerson, Jeff Schank, and Tim Waring for comments and conversation concerning the topics discussed in this manuscript. I also thank the anonymous reviewers for their helpful comments. This work was supported by NIH Pioneer Award DP1OD003874, awarded to J.M. Epstein.

\section{NOTES}

1. In some social insect colonies, the presence of multiple foundresses leads to low degrees of genetic relatedness. Nevertheless, control of differentiation and organization is still genetic in nature.

2. In evolutionary theory, "ultimate" explanations of a trait deal with the adaptive properties that caused natural selection to favor that trait, whereas "proximate" explanations deal with the immediate physiological and developmental mechanisms that give rise to the trait in an organism. Laland et al. (2011) discuss how feedback between organism and environment complicates this distinction.

3. Both Read (1958) and Ridley (2010) make compelling cases for the complexity and interconnectedness of technologies, but they also conclude that this complexity supports the case for unconstrained free markets, which I do not believe follows necessarily from their premises.

4. The adaptive fitness of group-level traits, as with individuallevel traits, is of course dependent on the selective environment. In a densely treed forest, the organizational properties of the Roman Legion may hinder rather than help, leading to domination by less encumbered Barbarians.

5. Explanations for the loss of complex technology in Tasmania have been proposed that do not rely explicitly on group-level traits, but instead rely on success-based biases in social learning (Henrich 2004b; Powell et al. 2009). Nevertheless, the arguments made in this target article imply that group organization and differentiated roles are crucial (and missing) parts of the Tasmanian story.

\section{Open Peer Commentary}

\section{Maintenance of cultural diversity: Social roles, social networks, and cognitive networks}

\author{
doi:10.1017/S0140525X13002811
}

\section{Marshall Abrams}

Department of Philosophy, University of Alabama at Birmingham, Birmingham, AL 35294-1260.

mabrams@uab.edu

http://members.logical.net/ marshall

Abstract: Smaldino suggests that patterns that give rise to group-level cultural traits can also increase individual-level cultural diversity. I distinguish social roles and related social network structures and discuss ways in which each might maintain diversity. I suggest that cognitive analogs of "cohesion," a property of networks that helps maintenance of diversity, might mediate the effects of social roles on diversity.

As Smaldino notes, cultural diversity matters because it is one source of a group's ability to generate solutions to challenges. He suggests that the patterns that give rise to group-level cultural traits can also increase individual-level cultural diversity (sect. 4.1, para. 6). Smaldino distinguishes between two dimensions of collaborative interdependence: social differentiation between individuals' characteristics and social organization-the fact that differentiation contributes to individuals playing different roles in collaborative tasks.

Although differentiated social roles in a collaborative process may depend on differences in individuals' characteristics, social roles need not require different characteristics. The same person may play the role of juror in one trial, witness in another, and defendant in a third. Moreover, some social roles, such as serving on a jury, do not require special characteristics. On the other hand, differences in social roles usually result in differences in experience. Thus, social roles may themselves generate diversity in patterns of thought and behavior, as suggested by Smaldino's remarks.

Social organization can encourage or constrain social network structure. To continue the earlier example, the amount and nature of communication between participants in a courtroom depends on their roles in the proceedings. Work within more persistent organizations often creates more persistent patterns of social networks. Social network models provide one way of characterizing certain differences between social roles by capturing differences in the direction, amount, and nature of what is communicated. Managers and employees both talk to each other, but the kinds of things they say may differ, and what each says to employees with different roles differs. This is exactly the kind of difference that can affect cultural transmission at the individual level, as illustrated, for example, by empirical studies of cultural transmission networks (Atran \& Medin 2008; Henrich \& Broesch 2011).

Thus, collaborative interdependence may influence (a) individual cultural diversity and (b) patterns of cultural transmission. Moreover, we can distinguish the causes of these two effects of collaborative interdependence: two organizations might have different social roles but very similar social network structures, 
Commentary/Smaldino: The cultural evolution of emergent group-level traits

or similar social roles but different social structures (juries and managers may have different roles in different kinds of court proceedings or in different companies).

Some patterns of cultural transmission tend to reduce cultural diversity. These patterns include copying of random individuals (Alexander 2007; Abrams 2013), bias toward copying successful or high-status individuals, and bias toward copying high-frequency cultural variants (Boyd \& Richerson 1985). Models in which individuals' beliefs are weighted averages of others' generally produce consensus, as long as no individuals are isolated from others and degrees of trust are stable (DeGroot 1974; Grim et al. 2011; Lehrer \& Wagner 1981; Zollman, personal communication, cf. Zollman 2013). Some models can maintain diversity, however.

A coordination game models a situation in which it is better for each person to do the same as others, even if one coordinated action might be preferable to another. In one kind of model, each person makes a single choice in simultaneous games with each neighbor on a network with bidirectional, unweighted links. The best response to neighbors' choices depends on the percentage of them who play various options, and the payoffs for coordination with each. Iterating the process can spread a given choice: if enough of my neighbors adopt, say, option A, I will do the same, which may eventually cause a neighbor who has been playing $B$ to switch to $A$, if her neighbors switch as well. This models cultural transmission in which individuals are influenced both by the number of neighbors adopting a variant and its perceived intrinsic attractiveness.

Morris (2000) showed that distinct cultural variants can be maintained in such a model if some groups have high enough cohesion, also known as cohesiveness (Vega-Redondo 2007; Young 1998). The cohesiveness of a group is the minimum of the ratio between the number of intra-group links and the number of all links, in each group member's links. A group with high cohesiveness is one with mainly within-group communication. Members' interactions reinforce their common cultural variants despite attractive alternatives presented by outsiders.

Alexander (2007) investigated agent-based simulations of various combinations of (a) games and payoffs, (b) network structures, and (c) rules for copying from neighbors. Some combinations of these factors make it difficult or impossible to maintain cultural diversity; others make it easy. Alexander's results appear to show that on the whole, more social interconnections tend to make cultural disagreement less likely, thus apparently broadening Morris's result to a variety of other cases.

Morris's sense of cohesion is related to others (Wasserman \& Faust 1994) and to measures of community structure (Newman 2010). Using a cohesion measure called close-knittedness, Young (1998) proves results for stochastic coordination games that support the idea that cohesion allows local reinforcement and global disagreement (at least in the short run). My own experiments with a simple model of cultural transmission that does not use averaging for updating of beliefs (http://modelingcommons.org/browse/one_model/3829) suggest that cultural diversity can easily be maintained by cohesion on reasonable timescales.

Plausibly, the influence of collaborative interdependence on social network structure sometimes generates structures in which groups exhibit cohesion, communicating largely with members of the same group on certain topics of discussion. This is a second way that collaboration can help maintain cultural diversity within a population.

As suggested by some of Durham's (1991; 1992) proposed transmission isolating mechanisms mentioned by Smaldino (sect. 6, para. 1), individual resistance to alternative variants or certain kinds of interlocutors can maintain cultural diversity (Axelrod 1997; Hegselmann \& Krause 2002; Mueller et al. 2010). Note though that cognitive processes such as inference and emotion can transfer influence from one mental state to another, and people seem to be quite capable of keeping large domains of thought isolated from others. This suggests that resistance to alternatives might be modeled in terms of cohesion-like properties of networks of influence between mental states (cf. Colombo 2013). Then different social roles may encourage "cohesive" cognitive subnetworks, reinforcing particular patterns of thought in individuals who fill similar social roles. Perhaps cohesion mediates both effects of collaborative interdependence on cultural diversity highlighted above.

\section{The primacy of scaffolding within groups for the evolution of group-level traits}

\section{doi:10.1017/S0140525X13002823}

\section{Linnda R. Caporael and Colin K. Garvey \\ Department of Science and Technology Studies, Rensselaer Polytechnic Institute, Troy, NY 12180. \\ caporl@rpi.eduｇarvec@rpi.edu \\ http://www.rpi.edu/ caporl}

Abstract: Although both a "simple dyad or a population of thousands" are groups, these are, respectively, face-to-face embodied groups and collective symbolic groups. We applaud Smaldino for recognizing and describing the concept of the group-level trait. As an expansion, we propose an evolutionary-developmental model of face-to-face groups that scaffolds theorizing the evolution of cultural group-level traits.

One look at the human body - bipedal, no claws, pitiful canines, and a long developmental period-and it is clear that humans could have only evolved from groups, not from a primal condition of solitary living. Members of other ancestral species, not even bipedal, faced the initial problems in the evolution of coordinated activity. Group-living evolved as an interface between individual and habitat (e.g., protection from predators; exploitation of large, patchy, distributed resources). Groups that formed more coordinated units to interact with the habitat (thereby benefiting component individuals) would have been relatively more persistent than less coordinated units. Both minimum and maximum constraints on group size would occur: eventually too small a group would have a higher risk of perishing; too large a group strains the carrying capacity of the environment. Because a group mediates individual contact with the environment, and the number of niches within groups is constrained by minimum group size and carrying capacity of the habitat, we expect the evolution of perceptual, affective, and cognitive processes that support the development and maintenance of group membership (Caporael et al. 1989). In short, we expect humans to have evolved to be obligately interdependent, unable to reproduce and survive to reproductive age outside a group. Few would disagree with this minimalist scenario. The point of traction is how we conceive of group structure, social motives, and cognition generally. We agree with Smaldino that more highly coordinated groups are likely to outcompete less coordinated ones, but we also emphasize that within-group pressures have the major role in evolved grouplevel traits, not between-group conflicts.

Anthropologists identify three categories of functional group organization among hunter-gatherer groups: hunting and gathering is typically done by workgroups, subdivisions of a band. A band undertakes domestic functions - butchering, preparing food for storage, child-rearing, and adjudicating conflict. A macroband is a seasonal gathering of bands, with a wide range of ritual, social, cognitive, and informational activities. The organizational structure is remarkably stable through time, across continents, and different habitat types. Independently, Hull (1988) observed similar configurations in his participant-observation research on the organization of scientific practice. He identified a "demic structure of science" consisting of small research groups, "conceptual demes," and seasonal society meetings. Group size at these three levels was comparable - about 3 to 5, 30 to 50, and 100 to 500 individuals (cf. Dunbar 1993), respectively. With the addition 
Commentary/Smaldino: The cultural evolution of emergent group-level traits

Table 1 (Caporael \& Garvey). Repeatedly Assembled Core Configurations

\begin{tabular}{|c|c|c|c|}
\hline Core configuration & Group size & Modal tasks & Group-level traits \\
\hline Dyad & 2 & $\begin{array}{l}\text { "Up-close" interactions; sex, artifacts, infant- } \\
\text { caregiver interaction }\end{array}$ & Microcoordination \\
\hline Task group & 5 & $\begin{array}{l}\text { Foraging, hunting, gathering, direct interface } \\
\text { with habitat }\end{array}$ & Distributed cognition \\
\hline Deme (Band) & 30 & $\begin{array}{l}\text { Movement from place to place, general } \\
\text { processing and maintenance, work group } \\
\text { coordination }\end{array}$ & $\begin{array}{l}\text { Shared construction of reality (includes } \\
\text { indigenous psychologies), relational group } \\
\text { identity }\end{array}$ \\
\hline Macrodeme (Macroband) & 300 & $\begin{array}{l}\text { Seasonal gathering, exchange of individuals, } \\
\text { resources, and information }\end{array}$ & $\begin{array}{l}\text { Stabilizing and standardizing language; } \\
\text { ontologies, collective identities }\end{array}$ \\
\hline
\end{tabular}

The names of core configurations refer to distinctive kinds of situated activity. The term "bands" is used to refer to (idealized) hunter-gatherers; otherwise, "deme" refers to the model. Except for dyads, the group size numbers should be considered as basins of attraction. Reprinted and modified from Caporael (2014).

of the dyad, considerations of repeatedly assembled morphology, tasks, and group size motivate a model of core group configurations that form a sociocognitive selective environment. This selective environment scaffolds both MLS and cMLS approaches, providing far richer possibilities for theoretical development and elaboration consistent with Smaldino's concept of the grouplevel trait.

Table 1 summarizes the model of core configurations (Caporael 2014; see also Caporael 1997). Core configurations are associated with examples of modal tasks that scaffold the evolution and development of group-level traits (Caporael et al. 2014). The model generally posits that human mental systems should have evolved for core configurations; once evolved these can be combined, extended, and co-opted to novel tasks. Selective advantages for sociality include coordination of activity and the acquisition, transmission, and maintenance of information and knowledge.

Seasonal macrodemes, composed of related demes, should be particularly active sites in human biocultural evolutionary studies. First, macrodemes are not persistent; they are intermittent over time. Second, members of groups may come and go within demes without changing the group structure and dynamics at the deme and macrodeme levels (Brewer \& Caporael 2006). The situated activity of hunting, foraging, playing, and other activities at deme and macrodemes differ little from each other. However, macrodemes generate a new set of dynamics, which are largely social and psychological with downward causal consequences. These emergent abilities include distributed cognition, reduced distinctions between self and non-self, and collective and categorical identities, rather than just interpersonal and relational identities. The interpersonal relational identity within bands is complemented by an emergent collective identity at the macroband level. Collective identity in a seasonal macrodeme scaffolds exchanges of crucial information about changing conditions in more distant parts of a local ecology. Language, and its stabilization and standardization, is highly significant for describing what lies beyond the next hill. Layton and O'Hara (2010) report that modern languages universally include the equivalent of terms such as "now," "before," "after," "here," and "far." A lack of such not-present ecological information can lead to the loss or even partial destruction of a foraging party, which in turn can lead to the end of a band. Although that end may be the literal deaths of its members, participation in a macroband with a shared collective group identity can scaffold the absorption of surviving group members by other demes. In other words, macrodemes serve as a safety net at the band and individual levels. Furthermore, the seasonal aspect of macrodemes, combined with shared symbolic communication among demes, scaffolds the transition from foraging lifeways to settlement living.
Although we do not disagree with Smaldino that intergroup competition and conflict occur, we are skeptical about using such traits as lynchpins for the evolution of a distinctive "human nature." It takes a great deal of within-group selection for the evolution of the kind of coordinating capacities demanded by engagement in inter-group conflict, even at the level of a minor mêlée. By the same token, intrademic, individual, and subgroup competition in various symbolic, ritual, and occasionally embodied ways, is a lively and refined sport.

\section{ACKNOWLEDGMENTS}

We thank Emily Schultz for helping us pull chestnuts out of the fire.

\section{Tackling group-level traits by starting at the start}

doi:10.1017/S0140525X13002835

Maciej Chudek ${ }^{\mathrm{a}}$ and Joseph Henrich ${ }^{\mathrm{b}}$

aschool of Human Evolution and Social Change, Arizona State University, Tempe, AZ 85287; ${ }^{\mathrm{b}}$ Department of Psychology, University of British Columbia, Vancouver, BC V6T 1Z4, Canada.

Maciej.Chudek@asu.edu

http://abcs.asu.edu/Maciek/

joseph.henrich@gmail.com

http://www2.psych.ubc.ca/ henrich

Abstract: We agree that emergent group-level properties are important; however, we disagree that current approaches, especially culture-gene coevolutionary (CGC) approaches, have neglected them. We explain how CGC helps demystify the tumult of humans' group-level complexity by "starting at the start," and why (a) assuming undifferentiated individuals and (b) focusing on cooperation are actually powerful tools to this end.

The culture-gene coevolutionary (CGC) approach recognizes the importance of emergent, group-level properties. CGC focuses of the evolutionary causes and consequences of our species' capacity to transmit complex cultural information, including the emergence of complex, differentiated, interacting phenotypes that no single individual could have developed in isolation.

Once culture began evolving, fascinating new evolutionary dynamics emerged. These led our ancestors to conform (Boyd \& Richerson 1985), imitate prestigious leaders (Henrich \& GilWhite 2001), differentiate into symbolically marked ethnic 
groups (McElreath et al. 2003), differentiate into specialized economic roles within those groups (Henrich \& Boyd 2008), and instantiate complex cooperation-sustaining ecological dynamics and institutions (Chudek \& Henrich 2011). These institutions underwent their own interactions and evolutionary dynamics, leading eventually to Roman legions and stock markets. CGC investigates how these dynamics are shaped by two interacting systems of inheritance: genetic and cultural.

From shamans - who magically influence others' fortunes - to hipsters - who dislike music once it becomes popular - it is hard to think of a human phenotype that is not a specialized adaptation to the emergent institutions and specialized roles of their peers. Contemporary human societies are a brilliantly complex interplay of emergent roles, institutions, technologies, and the socially transmitted concepts and worldviews that support them. We sympathize with Smaldino's wanting to draw attention to such collective traits. After all, some social scientists have an epistemic commitment to exclusively individual-level explanations. However, we do not think that making sense of this complex emergent domain is nearly as simple as Smaldino implies, and it is certainly harder than merely acknowledging its importance. In fact, the CGC approaches that Smaldino criticizes for neglecting emergent traits are some of our best efforts to understand them.

There are two ways scientists can make headway into this emergent cacophony. We could start at the end. We could recognize the existence of group-level traits, measure them, and see what effects they have, without worrying too much at first about where they come from. This is what functionalist anthropology and sociology did for many decades.

More recently, cultural psychologists have also started at the end. In cultural psychologists' experimental designs and statistical models, group-level collectives, "cultures," (e.g., "East Asian" and "Euro-American" cultures) are, by assumption, fixed, group-level, dichotomous or categorical predictors of individual phenotypic differences. This supposition that group-level traits both exist and shape individual behavior continues to reveal surprising and subtle psychological phenomena (Heine 2012).

The CGC approach is to make sense of the emergent maelstrom of contemporary human societies by starting at the start. Launching from our best descriptions of the world before these complex group-level traits emerged, we reconstruct the underlying individual-level interactions that brought them to be. Starting from models of undifferentiated individuals, we show how early kinds of social differentiation could emerge, such as ethnicities, economic stratification, or individuals differentiated by prestige. Next, we make sense of how these within-group interactions give rise to stable group-level norms and maintain them in spite of migration. Equipped with an understanding of why group-level properties exist, and the individual-level underpinnings of how they emerge, survive, change, and dissipate, we can begin to postulate explanations of their interactions and histories (e.g., Henrich 2009; Henrich et al. 2012; Norenzayan 2013).

Smaldino's suggestions that we move "beyond cooperation" also misses the value of existing work, which already includes work on emergent phenomena like marriage (Henrich et al. 2012), ethnic groups (McElreath et al. 2003), divisions of labour (Henrich \& Boyd 2008), rituals (Atran \& Henrich 2010) and "innovationenhancing institutions". For example, Smaldino points out that the effects of sociality on technological complexity, captured in Henrich's "Tasmanian model" (2004b), also apply to understanding the effects of different institutions. Oddly, Smaldino seems to contrast his seemingly novel point with the perspective of culturegene coevolutionists, including Henrich; but Henrich has made this point repeatedly (e.g., Henrich 2009).

From most vantages, self-differentiating humans are very complex. To build on Smaldino's example, Roman legionnaires not only came from different ethnic groups, but they also shopped at markets for goods traded through continent-spanning networks, bemoaned intricate local politics, frequented prostitutes, vilified minorities, blamed supernatural agents for disasters, gave alms to street urchins, cast magic spells to harm others, contributed to public works, sometimes by coercion and sometimes for pay, and tried to incorporate strange foreign ideas into their developing worldviews. Are there simple insights and theorems that can make sense of the tumult of even a single legionnaire's phenotype? Are we doomed to unintegrated social-scientific micro-theories of each dimension along which individuals differentiate?

The power of cooperation is that it lets us squint our eyes and rotate our vantage until we are looking at a dimension that (a) permeates everything, at all scales; (b) tends to have powerful consequences, and (c) usually obeys an orderly set of principles that we can reason about mathematically. Cooperative dilemmassituations where individuals can gain less (or suffer more) so that others gain more (or suffer less)-are ubiquitous. They arise in interactions among genes, individuals, groups, species, ideas, cultures, institutions, and on and on. Whenever they do arise, they share important properties that we are rapidly coming to understand. Criticizing this emerging understanding for abstracting away the details of the differences between individuals is like criticizing theories of heat for invoking a single scalar rather than trying to model the trajectories of every atom in a gas. It confuses a powerful theoretical feature for an accidental omission.

It is one thing, a valuable and worthwhile thing, to acknowledge complex group organization and behavior. We support Smaldino in encouraging it. It is an altogether more difficult thing to formalize, model, and ultimately to explain that complexity. The approaches Smaldino accuses of ignoring the group-level complexity are actually a principled effort to understand it by starting at the start, rather than in the middle. We wonder what specific psychological, cultural, or historical questions-which have eluded other researchers - Smaldino has tackled with his approach?

\section{The priority of the individual in cultural inheritance}

\section{doi:10.1017/S0140525X13002847}

\section{Taylor Davis and Eric Margolis}

Department of Philosophy, University of British Columbia, Vancouver, BC V6T 1Z1, Canada.

\section{taylor.thiel.davis@gmail.com}

http://www.taylordavisphilosophy.com

eric.margolis@ubc.ca

http://www.margolisphilosophy.com

Abstract: Smaldino's proposed extension of the theory of cultural evolution embraces emergent group-level traits. We argue, instead, that group-level traits reduce to the traits of individuals, particularly when it comes to the question of how group-level traits are inherited or transmitted, and that this metaphysical fact is integral to the theory of cultural evolution.

Smaldino takes the concept of emergent group-level traits to be a "conceptual extension of the theory of cultural evolution," (abstract) especially as cultural evolution is conceived by Richerson and Boyd and their collaborators in what Smaldino calls cMLS theory (see, e.g, Richerson \& Boyd 2005). But he also suggests that this conceptual extension involves treating grouplevel traits as somehow autonomous from, or irreducible to, individual-level traits. For example, he claims that group-level traits "present a unit of cultural selection that is not encompassed by selection on individuals" (sect. 1, para. 2). We see no inherent problem with the notion of group-level properties that cannot be reduced to the properties of individuals. However, not all of the properties of a given group (or individual) are heritable, and only heritable properties can be understood as traits explained 
by principles of selection. This creates a problem, because the cMLS framework is fully committed to a notion of inheritance that makes sense only at the individual level, and individuals cannot inherit group-level properties.

The cMLS framework is based on the assumption that cultural learning can be viewed as a form of inheritance, a nongenetic mechanism by which one individual comes to possess the same trait as some other individual. This makes it possible to measure the frequencies of cultural traits in populations of individuals, which in turn makes it possible to explain changes in those frequencies over time. These frequencies are precisely what evolves in cultural evolution; the basic aim of evolutionary theories based on population modeling - both cultural and genetic - is to explain frequencies in populations.

Yet it makes no sense to ask, for a given group, about the frequencies of its group-level properties. To calculate frequencies one must count individuals, and individuals as such cannot possess group-level properties. As Smaldino emphasizes, for a Roman group to be more organized than a Barbarian group is not for the Roman group to have a greater frequency of individuals who possess the trait of being organized. Organization is not a trait possessed by any member of the group, so it makes no sense to ask how many group members possess the trait.

Moreover, the distinction between genetic evolution and cultural evolution is based on the prior distinction between genetic inheritance and inheritance by learning, and these are facts about how individuals acquire traits. To abstract away from facts about inheritance at the individual level is to abstract away from precisely those facts that allow us to distinguish cultural evolution from genetic evolution. This will obviously be a problem for any attempt to appeal to emergent group-level traits in accounts of gene-culture coevolution, because these accounts require us to keep track of the distinct contributions made by cultural evolution and genetic evolution.

Of course, patterns of organization and differentiation may be maintained and preserved within groups for long periods of time, across many generations. But the cMLS framework can fully explain this in terms of facts about inheritance among individuals. Suppose a singer wants to form a band that sounds like the Beatles. What would it take for a group to imitate this group-level property? Fortunately, it would be sufficient to find a drummer who imitates Ringo and, separately, a bass player who imitates Paul. There is no need to find a drum-andbass duo who learned together to imitate the Beatles' rhythm section. We can explain the cultural inheritance of the Beatles' distinctive sound by explaining how its individual members copied the distinctive styles of individual members of the Beatles.

Or consider Smaldino's own example of the Inuit method for harvesting eggs. Here, two differentiated collaborators are engaged in a culturally inherited practice, but this does not entail that a group (a dyad) has inherited a group-level property. Rather, it entails only that individuals who perform separate roles have each learned, separately, how to play their roles. When a rope-holder learns from a cultural model how to tie a certain knot, and when an egg-gatherer learns from a cultural model which eggs are the best ones, what gets inherited are only those properties that the model and the learner share. Differences between individuals - differentiation-may re-emerge in the new generation. But it does so only after the events of inheritance have taken place at the individual level.

To make sense of group-level properties within the cMLS framework, then, we must resist the temptation to think of grouplevel properties as autonomous group-level traits-heritable properties that groups may possess regardless of facts about their individual members' histories of cultural learning. Smaldino seems to have succumbed to this temptation:

The cargo cultists appear to have gotten the idea that, in order to receive cargo, they needed to do more than adopt particular behaviors - they needed to adopt particular social structures. This is significant, because social transmission of behavior is typically assumed to occur at the level of individuals. Here instead we see the transmission of social organization. (sect. 6.1 , para. 2)

For the reasons just given, it is a gross mischaracterization of the cMLS framework to say that social transmission is "typically assumed" to occur at the level of individuals. This is not an optional feature of the theory; to drop it would be to step outside of the cMLS framework altogether.

As long as we know which individual-level facts constitute the "inheritance" of group-level properties, the term group-level inheritance may be a harmless way of speaking loosely. But to talk of group-level inheritance instead of individual-level inheritance, as Smaldino does here, is to imply that there is no need to map group-level inheritance onto prior and more basic facts about individual-level inheritance. That is not to offer a conceptual extension of the theory of cultural evolution. It is to offer a different kind of theory altogether, a theory that for principled reasons cannot be integrated into the existing theory of cultural group selection.

\section{Studying the emergence of complicated group- level cultural traits requires a mathematical framework}

\author{
doi:10.1017/S0140525X13002859
}

\section{Michael Doebeli ${ }^{\mathrm{a}}$ and Burton Simon ${ }^{\mathrm{b}}$}

${ }^{a}$ Department of Zoology and Department of Mathematics, University of British

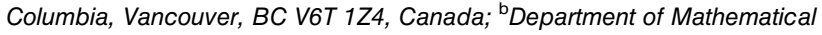
and Statistical Sciences, University of Colorado at Denver, Denver, CO 80202. doebeli@zoology.ubc.caßBurt.Simon@ucdenver.edu

\section{www.ubc.ca http://www.ucdenver.edu}

Abstract: Understanding the cultural evolution of complicated grouplevel traits requires the mathematical formulation of a dynamical system with birth and death events at multiple levels, that is, at the level of individual humans and at the level of groups of humans. Both levels are characterized by cultural traits that have complicated transmission, innovation, and inheritance mechanisms and that can undergo a form of Lamarckian evolution.

The article by Smaldino raises an important issue in cultural evolution: How can the properties emerging from a division of labour between members of a group be seen as properties of that group? In all organisms, different parts perform different tasks for the benefit of the whole organism. For example, in single-celled bacteria, many different molecules are involved in nutrient uptake, which can be viewed as an emergent property of the organism. Similarly, in animals, properties of the circulatory system can be viewed as emerging from the concerted operation of many different groups of specialized cells. In bacteria and animals, the evolution of these kinds of emergent properties is studied based on the principles of genetic inheritance.

However, in human societies, many types of emergent properties, like the ones described by Smaldino, arose only recently and almost certainly have no genetic basis other than the genetics underlying cognitive abilities in humans, which likely remained essentially unchanged in recent times. Therefore, in human cultures understanding the evolution of emergent group properties requires an evolutionary theory that is not based solely on genetic inheritance.

We agree with Smaldino that, even though there is consistent pushback against viewing culture as something that evolves akin to "Darwinian selection," the theory of cultural evolution originated by Boyd and Richerson (1985) is here to stay. Smaldino claims that the kind of cultural evolution he has in mind, that is, the evolution of emergent group-level traits, "is not fully 
accounted for by the multilevel selection perspective currently in vogue" (sect. 1, para. 3). We tend to agree, but we also note that Smaldino does not provide a feasible alternative, that is, a mathematical framework for a comprehensive multilevel theory that accounts for emergent group-level properties in the context of cultural evolution. Here, we would like to propose how one might construct such a framework.

From a modelling perspective, a multilevel evolutionary theory requires birth and death processes at different levels. In particular, to study group selection, one needs birth and death events not only at the level of individual organisms, but also at the level of groups, where group births result from fissioning and group deaths result from extinction and (perhaps) absorption by other groups. We have described such a general mathematical theory of group selection in Simon et al. (2013). Identifying group-level events is important, because it requires the identification of a unit (i.e., the "group") whose properties (emergent or otherwise) impinge on the unit's birth and death rates. In his verbal theory, Smaldino does not properly identify such units with their corresponding birth and death events. He mentions, for example, that some groups, such as armies, only exist ephemerally and hence cannot be viewed as units of selection. But surely the complicated organization of an army should not be viewed as a cultural property of the army itself, but instead as a property of the society that is represented by the army, which may be a much less ephemeral group. In section 6.2 Smaldino also mentions how groups with different types of religions might enjoy different levels of success when the different groups are competing, but again, in the absence of an explicit description of the group level events, the arguments remain vague.

The group selection theory in Simon et al. (2013), based on genetic inheritance, provides a possible starting point for modelling cultural evolution. However, that theory needs to be modified, because cultural transmission, innovation, and inheritance are different from genetic inheritance. In particular, cultural evolution has some telling signs of a Lamarckian process, for example, the tendency for cultural traits to change during an individual's lifetime and the inheritance of those acquired traits by its offspring. We point out that this important aspect of cultural evolution is not commonly acknowledged (but see, e.g., this video of Steven Pinker's talk in the Harvard Mind/Brain/Behavior Distinguished Lecture Series, April 21-23, 2009: http://www.youtube. com/watch?v=6XT_-NSfujw\&list=PLFA5D03203B10A14F). In the group selection theory of Simon et al. (2013), feasible modifications can allow a form of Lamarckian evolution at the individual (within-group) level, as described in Simon (2008), and additional variables at the group level to account for emergent cultural traits. The population in the model would consist of various groups of individuals (tribes, societies, etc.), where in each group, individuals have cultural traits that may change over their lifetimes as a result of learning. The learning process may be simple (e.g., learn from the "best") or complicated (e.g., gauge the distribution of cultural behaviours and try to predict success). When individuals have children, these children tend to "inherit" (i.e., copy) the culture that their parents have at the time they are born, and hence not necessarily the culture that the parents had at the time they themselves were born. These are the Lamarckian aspects of the process. Culture can of course also be transmitted horizontally, both within and between groups, and culture is also passed vertically at the group level through fissioning events and horizontally at the group level through absorption events.

The concrete details of a given model would depend on the situation the modeller had in mind, but the general setup would be similar to that in Simon et al. (2013). In the simplest version of such models for cultural group selection, individuals would not vary genetically, so that only culture evolves. Such models could be extended to incorporate genetic differences between individual humans, for example in the ability to absorb cultural information, thus potentially also providing a framework for studying the genetic evolution of cognitive machinery, as, for example, envisaged in the norm psychology framework (Boyd \& Richerson 2009a; Chudek \& Henrich 2011).

As the article by Smaldino makes clear, cultural evolution is in general a complicated, multilevel process, and some cultural properties can only be defined at the group level. In the light of such complexity, it is of paramount importance to develop a mathematical framework in which all the variables, as well as the rules by which they change, are explicitly spelled out. Such models will probably show that cultural evolution, and in particular the evolution of group-level cultural traits, is a complicated process that can exhibit qualitatively different dynamics from genetic evolution because of the different mechanisms of transmission and inheritance.

\section{Feedback, group-level processes, and systems approaches in human evolution}

\author{
doi:10.1017/S0140525X13002860
}

\section{Agustin Fuentes \\ Department of Anthropology, University of Notre Dame, Notre Dame, IN 46556. afuentes@nd.edu}

http://anthropology.nd.edu/faculty-staff/fuentes_agustin/index.shtml

Abstract: An explicit role for emergent group-level traits, social role diversification, and coordination makes good sense when thinking about human evolution. To most effectively understand these and other facets, we need to move toward an integrative model that assumes niche construction and encompasses positive feedback systems at individual, subgroup and larger group levels, demographic processes, and local ecologies.

Moving beyond a focus on the individual to think seriously about group-level processes is relevant not just for cultural evolution but for human evolution overall. We are at a point in our understandings of evolutionary and behavioral processes that suggests we move beyond the reliance on a focus on individuals, single events, and single traits to a systems approach, including group dynamics, structure, and emergent traits, in analyses of evolutionary histories and processes (Bateson \& Gluckman 2011; Hinde 1976a; Laland et al. 2000; Oyama et al. 2003; Sterelny 2012).

Incorporating niche construction and social, behavioral, and symbolic inheritance, in addition to ecological and genetic inheritances, should be central in our examinations of human evolutionary trajectories and processes (e.g., Dunbar et al. 2010; Fuentes 2013; Flynn et al. 2013; Henrich 2011; Jablonka \& Lamb 2005). An explicit role for emergent group-level traits, social role diversification, and coordination makes good sense.

Smaldino argues that human groups produce emergent grouplevel traits that result in between-group differences in fitness and are heritable. If this is the case, then we need not only to describe such patterns in modern humans, but also to develop a model where these patterns (and the capacities for them) could have evolved. A core for such a model lies in the recognition that increasingly dense conception and transmission of information alongside substantive neurological and behavioral plasticity characterize "the human socio-cognitive niche" (Whiten \& Erdal 2012) and that humans use a hybrid learning system where central social and material skills are acquired by combining information from the social world and the physical-biological world (Sterelny 2012). In short, modern human cooperation, collaboration, and coordination are related to organizational differentiation and to the semiotically and semantically rich human communication system, which evolved over the Pleistocene from simpler systems that had the potential to generate increased 
complexity. A full understanding of the role of group-level emergent traits requires a systemic explanation that is applicable (at least in part) across different members of our direct lineage over the past one to two million years.

In particular we need an explanation that has an explicit connection to the evolution of the genus Homo and its history of a "positive feedback loop that drove life history evolution and contributed to cultural change" (Anton \& Snodgrass 2012, p. S492). This feedback system was an iterative process where increasing brain size, dietary quality, and cooperative behavior results in lowered extrinsic mortality risk, changes in cognition, body composition, life history parameters, and behavioral and communicative complexity (culture) (Anton \& Snodgrass 2012; Sterelny 2012). We need to move toward an integrative model that encompasses positive feedback systems at individual, subgroup and larger group levels, demographic processes, and local ecologies (Fuentes 2013; in press). Smaldino's emphasis on development through overlapping cycles is a step in the right direction.

There is substantial evidence that behavioral and cultural inheritances play especially salient roles in evolutionary patterns and outcomes for the genus Homo, even before the emergence of fully modern Homo sapiens sapiens (Gamble et al. 2011; Henrich 2011; Kendal 2012; Richerson \& Boyd 2005). It was within the context of local and regional communities (comparable to the larger end of Smaldino's trait groups) that members of the genus Homo interfaced, interacted with, modified, and were modified by, social and ecological worlds during the course of our evolution (Fuentes, in press; Sterelny 2012). I suggest that a focus on evolutionary processes at multiple levels (individual, subgroup, and community) and the feedback and interfaces between these levels, across the Pleistocene, can facilitate a linkage between Smaldino's model of the centrality of emergent grouplevel traits in modern humans and the systems of evolutionary processes in earlier members of the genus.

Unfortunately, Smaldino's reliance on battlefield, corporate, and sports analogies as metaphors of choice and on inter-group competition as a central driving factor in the evolutionary processes obfuscates the development of such attempts. Direct conflict, business, and sports and military models are not the only, or the best, heuristic tools for thinking about evolutionary processes, especially over the majority of human evolutionary history. Using this format, even metaphorically, limits the explanatory toolkit and constrains perspectives in modeling dynamic histories. It also discounts, or overlooks, possibilities that drift, and evolutionary processes aside from selection may play substantive roles in shaping trajectories. Smaldino also assumes a kind of consistent selective progress wherein as cultural knowledge and behaviors move across time, better ideas and practices replace those that are less effective. Such an assumption of clear directional progress is not warranted in a larger view of human evolution. Much of our history as a genus is better seen as ebb and flow of innovation and extinction, of progress and retrogress, in technologies, behavior, and adaptation.

While I applaud expanding the focus of evolutionarily relevant actions beyond the individual, Smaldino's definition of a trait group as a collection of individuals interacting to produce a trait and the assertion that such a group may range from a simple dyad to a population of thousands is problematic. I agree that both dyadic and the large-scale interactions can be evolutionarily relevant, but I do not think these two things can be assumed to be functionally equivalent entities in an evolutionary model.

Finally, and importantly, Smaldino assumes that once cooperation has evolved, the persistent association, interdependence, and information transmission between individuals enable the evolution of increased complexity in collaboration, the differentiation of roles, and a more structured social organization. I agree and suggest that a high level of cooperation shows up relatively early and forcefully in human (even hominin) evolution (Fuentes 2013; Malone et al. 2012). Thus, our explanations for much of the current hypercomplex behavior and cultural processes in modern humans need not include explanations for the extant baseline of cooperation; rather they, as Smaldino does, need to take it as a jumping off point and strive to explain not the baseline but the changes, and potential emergent properties, that it facilitates.

\section{ACKNOWLEDGMENTS}

This commentary arises from research supported by the Center of Theological Inquiry's Inquiry on Evolution and Human Nature, The Dean of the College of Arts and Letters, University of Notre Dame, and The John Templeton Foundation award \#20926.

\section{What is a group? Conceptual clarity can help integrate evolutionary and social scientific research on cooperation}

\section{doi:10.1017/S0140525X13002872}

\section{Drew Gerkey ${ }^{\mathrm{a}}$ and Lee Cronk ${ }^{\mathrm{b}}$}

${ }^{a}$ Postdoctoral Fellow, National Socio-Environmental Synthesis Center, University of Maryland, Annapolis, MD 21401; ${ }^{\mathrm{b}}$ Department of Anthropology and Center for Human Evolutionary Studies, Rutgers University, New Brunswick, NJ 08901.

drew.gerkey@gmail.com

www.sesync.org

Icronk@anthropology.rutgers.edu

http://anthro.rutgers.edu/fac/department-undergrad-a-grad-faculty/lee-cronk

Abstract: Smaldino argues that evolutionary theories of social behavior do not adequately explain the emergence of group-level traits, including differentiation of roles and organized interactions among individuals. We find Smaldino's account to be commendable but incomplete. Our commentary focuses on a simple question that has not been adequately addressed: What is a group?

In answer to the question "What is a group?" Smaldino adopts Wilson's (1975) concept of a trait-group, defining groups according to how the interactions among individuals impact their fitness. This leads to groups that "may consist of a simple dyad or a population of thousands" (sect. 2.1). In addition to concerns raised by others (Okasha 2006; Sterelny 1996), we see two problems with using the trait-group concept. First, the generality of this definition - encompassing almost any kind of social interaction across vastly different scales - provides little guidance for the task of understanding how the many different kinds of groups found in human societies emerge. Second, because cultural group selection is defined as competition among groups that are defined in cultural terms, it requires us to define the groups in question in cultural terms, not biological ones. Scholars in the social sciences have devoted considerable effort to documenting and analyzing the diverse groups that individuals form to cooperate, coordinate, or act collectively, but links between this work and cultural group selection remain tenuous (Cronk \& Leech 2013). Drawing on insights from the social sciences, we propose two conceptual distinctions that complement Smaldino's ideas about emergent group-level traits.

Our first suggestion is a conceptual distinction between objective and subjective groups. Objective groups are those whose boundaries are shared by all members of the population, meaning the boundaries between members and non-members are the same from one individual to the next. Objective groups correspond most closely to the common understanding of the word "group" and reflect the definitions used by both advocates and critics of early theories of genetic group selection (Maynard Smith 1998). Subjective groups are those whose boundaries differ depending on one's position within the population, meaning that the boundaries between members and non- 
Commentary/Smaldino: The cultural evolution of emergent group-level traits

members will be different from one person to the next. For example, human kinship systems often feature both descent groups - with objective boundaries between lineage members and non-members - and kindreds - with subjective, ego-centric boundaries dividing relatives and non-relatives (Cronk \& Gerkey 2007). Although the distinction between objective and subjective groups in this example is dichotomous, in other cases it may be more of a continuum, with some groups displaying more or less objective boundaries than others.

An advantage of this approach to defining groups is that it can be used to establish clearer links between theories of multilevel selection and empirical research on individual/group dynamics in the social sciences. In particular, the growing interdisciplinary research program on quantitative social network analysis provides a wide range of conceptual and statistical tools for defining groups along the objective/subjective continuum, including clustering, centrality, and cliques, among others (Jackson 2008; Wasserman \& Faust 1994). Although some evolutionary scholars have begun to use these tools from network analysis (e.g., Apicella et al. 2012; Nolin 2011; Pacheco et al. 2008), we believe this approach deserves greater attention. This suggestion applies particularly to those who use some version of the traitgroup definition, because these "groups" are better understood as networks emerging from and in turn shaping the interactions of individuals. For example, there is consensus among evolutionary scholars that mechanisms of positive assortment are necessary for the evolution of cooperative behavior among individuals, but are different mechanisms (kinship, reciprocal contingency, reputation, adherence to cultural norms) more or less likely to lead to the emergence of objective or subjective group structures?

Our second suggestion is a conceptual distinction between corporate groups and categorical groups. As the name implies, corporate groups are functionally integrated and have clear, body-like organizational structures that coordinate the actions of their members. Categorical groups are composed of individuals who share a bundle of characteristics or traits that distinguish members from non-members, but these boundaries do not rely on the kind of organizational structures found in corporate groups. Our distinction between corporate and categorical groups shares some aspects of Smaldino's distinction between aggregate and emergent groups, particularly the emphasis on diversification and organization in the latter. However, our terminology has two advantages. First, it establishes closer connections to the large body of social science research on competition among corporate groups-including firms (e.g., Arthur 2012), political interest groups (e.g., Baumgartner et al. 2009), organized religions (e.g., Stark 1996), and descent groups (Keesing 1975) - and categorical groups - including those shaped by shared ethnicity (Barth 1969), spirituality (e.g., Fuller 2001), and nationality (Anderson 1991). Second, it builds on theories of cultural group selection to develop testable hypotheses about the relationship between group-level traits and the fitness consequences of interactions among individuals. For example, because categorical groups are not organized in any way, cultural group selection acting on such groups should result in traits that help the group's individual members achieve their personal goals rather than any group-level goals. One might refer to this as "soft" cultural group selection. In contrast, cultural group selection acting on corporate groups should result in traits that influence the group's ability to achieve its collective goals, even when this runs counter to its members' efforts to achieve their personal goals. Consider, for example, the fact that institutions frequently impose rules against nepotism to promote the interests of the corporate group over those of its individual members. One might refer to this as "hard" cultural group selection.

These suggestions for conceptual distinctions among different kinds of groups are not meant to be exhaustive. Rather, we believe they reflect important insights gained through the work of generations of social scientists who have explored the implications of Durkheim's famous argument - echoed in Smaldino's article: "society is not the mere sum of individuals, but the system formed by their association represents a specific reality which has its own characteristics" (1895/1964, p. 129).

ACKNOWLEDGMENTS

Drew Gerkey was supported by the University of Maryland and NSF Award \#DBI-1052875 to the National Socio-Environmental Synthesis Center.

\section{Group-level expression encoded in the individual}

\author{
doi:10.1017/S0140525X13002884
}

\section{Paul Ibbotson \\ Open University, Centre for Childhood, Learning and Development, Milton \\ Keynes MK7 6AA, UK. \\ paul.ibbotson@open.ac.uk \\ http://www.open.ac.uk/education-and-languages/main/people/paul. ibbotson}

\begin{abstract}
Smaldino proposes a conceptual extension to the theory of cultural evolution to include emergent group-level traits as a unit of selection. It is important to recognize the role that group-level traits have played in the evolution of human culture. However, the emergent group-level trait of division of labor provides an illustrative example that is implementable within the existing framework of cMLS theory and may not even need a departure from the standard model.
\end{abstract}

Group-level traits cannot be expressed by individuals (by definition groups are more than one individual); however, whether group-level traits can be encoded by individuals relates more directly to the main claim of the article: that we need a conceptual extension to the theory of cultural evolution to include emergent group-level traits as a unit of selection. The illustrative example of division of labor helps to examine this claim.

When discussing the division of labor in the context of grouplevel traits, Smaldino raises two questions that are supposed to be difficult to reconcile within standard cMLS framework: (1) "How are individuals selected for specific roles?" [within the group] and (2) "Where do the available roles come from in the first place?" (sect. 5.2). It is possible that both of these questions can be answered, in principle, at the level of the individual, for example (1) by showing that individuals can be selected for their role through a combination of a good-stay bad-shift strategy in combination with inherited variation in task-aptitude, and (2) by showing that naturally occurring problems of coordination provide affordances for different roles and need not be socially constructed as Smaldino appears to suggest. To expand on these points, say individuals behave in a certain way, which we can call a good-stay bad-shift phenotype. If individuals are good at a particular task (or at least better than their neighbor), they stay put. If they are bad (or worse than their neighbor), they shift what they are doing and try something else. Given some variation in the aptitudes for different tasks in a population, this strategy, defined at the level of individual, ensures the best person for the job by self-sorting.

One can see how this tendency could form the basis of a structured group. To use the example of hunting, one member of the group might be better at running, one member better at wrestling prey, one with better eyesight, one better at identifying different prey, and so on. The efficiency saving bought about by the division of labor (law of comparative advantage; Ricardo 1817) means that those groups with self-sort division of labor will tend to do better than groups that do not behave in this way. The social structure is expressed as a group (one cannot benefit by trading skills with oneself) but encoded at the level of the individual (the goodstay bad-shift strategy). Once this structure is in place, one can imagine second-order and third-order hierarchal roles becoming 
available-for example, coordinating leadership roles-but still emergent from lower-level properties of the group. To answer Smaldino's second question, where available roles come from in the first place, again consider the hunt example above. Importantly, the roles themselves need not necessarily come from existing human societal structure, as is implied with the examples used by Smaldino (although they might do). Organization emerges in the above example from the affordances of a hunt event and the self-sort strategy.

I agree that it is important to recognize that an organized and structured group is qualitatively different from an aggregation of individuals and an important unit in the evolution of human culture. (However, the distinction between aggregate and emergent systems might not be as straightforward as this suggests, captured by the complexity science aphorism: more is different). All of this is not to claim the reducibility of all group-level traits to the individual. More that, because the standard model is so powerful one needs to be sure to rule out individual-level explanations first (Sumpter 2006). In the division of labor examples above, the answers to both of Smaldino's questions can be sensibly grounded at the level of the individual. Moreover, because of Ricardo's law of comparative advantage, what is good for the group is also good for the individual. In this context, it might make sense to talk of the social structure that emerges from a good-stay bad-shift mutation as an extended phenotype (Dawkins 1989). If this is right, then it is not so clear that we need a conceptual extension to cMLS, or even that a between-levels perspective dissolves the equivalency between MLS and inclusive fitness theory, as Smaldino proposes.

\section{Culture as an aggregate of individual differences}

\section{doi:10.1017/S0140525X13002896}

\section{Kyungil Kim, ${ }^{\mathrm{a}}$ Joonghwan Jeon, ${ }^{\mathrm{b}}$ and Youngjun Park ${ }^{\mathrm{a}}$ \\ ${ }^{a}$ Department of Psychology, Ajou University, Woncheon-dong, Yeongtong-gu, Suwon, Korea 443-749; ' Humanitas College, Kyung Hee University, Yongin, Korea 446-701. \\ kyungilkim@ajou.ac.kr \\ http://ajou.ac.kr/ tetross \\ evopsy@gmail.com_cyber13@ajou.ac.kr \\ www.khu.ac.kr}

\begin{abstract}
We question Smaldino's argument that culture plays the active role of maintaining and transmitting social organizations of differentiated individuals. Culture is an aggregate of individual differences in psychological variables within and between groups; it was not designed by group-level selection to maintain the structured organization of individuals. We conclude that Smaldino fails to present the crucial mechanism by which group-level traits are maintained, transmitted, and evolve.
\end{abstract}

Smaldino proposes that emergent group-level traits can be maintained and evolve by culturally transmitted norm-enforcing mechanisms such as narratives, mythologies, and religion. Such mechanisms can maintain the structured organizations of differentiated individuals because, as Smaldino claims, culture can have important influences on how individuals in a culture perceive any given set of situations. We argue, however, that culture cannot be regarded as an autonomous, superorganic force shaping human psychology. By finding other individual differences correlated with cultural differences, one can properly eliminate the explanatory power of culture in psychological models. We thus suggest that Smaldino fails to present the crucial mechanisms by which group-level traits are transmitted and evolve.

One goal of the target article is to document mechanisms by which emergent group-level traits are maintained, transmitted, and evolve. Based on Nisbett and his colleagues' evidence for cross-cultural differences in cognitive performance (Nisbett \& Miyamoto 2005; Nisbett et al. 2001), Smaldino contends that culture can constrain how individuals within groups perceive situations in a way that norm-enforcing mechanisms such as narratives, mythologies, and religion would reinforce norms of social organizations. Note that in this avenue of cultural psychology, culture is deemed to be an autonomous, external variable that independently affects cognitive processing by members of that culture. Different thinking and behavior between members of East Asian and Western cultures (e.g., holistic vs. analytic cognition) are alleged to result from the fundamental differences in cognitive architecture formed over thousands of years of cultural development (Nisbett et al. 2001).

Although culture statistically affects cognitive performance, it does not follow that culture is a superorganic force that transcends an aggregate of individual differences within a culture. Put it differently, Nisbett and his colleagues' (2001) model of holistic versus analytic thinking should not be construed as indicating fundamental differences in modes of thought between Easterners and Westerners. For example, European Americans are not more individualistic than African Americans or Latinos and are not always less collectivistic than Japanese or Koreans. Among Asians, only Chinese show large differences with Westerners, being both less individualistic and more collectivistic than Americans (Oyserman et al. 2002). National averages of culture-related personality factors are often unstable as a result of, among many others, the reference-group effect, a tendency for people to respond to self-report items by comparing themselves with the implicit standards of their own culture (Heine et al. 2008).

An alternative and more fruitful approach is to identify psychological variables that ultimately drive individual differences in cognitive processing and are sources of variation in performance of different cultural groups. Only when we are unable to identify such variables should we conclude that members of different cultures are qualitatively different at the level of their cognitive architecture (Kim \& Markman 2006; Weber \& Hsee 2000). Thus, we suggest that psychological variables that are correlated with cultural differences can lead to patterns of behavior in cognitive tasks that are like those observed in cross-cultural studies. For example, Peng and Nisbett's (1999) cross-cultural study reported that East Asians showed a greater preference for dialectical proverbs than did Americans. Kim and Markman (2006) explained these cross-cultural differences using a universal motivational variable, fear of isolation (FOI). They found that inducing a high level of FOI in Americans made their cognitive performance become more similar to that of East Asians, as reported in previous cross-cultural studies (Peng \& Nisbett 1999). Koreans had a greater relative preference for dialectical proverbs than Americans had. Statistical analyses showed that the between-culture variation in preference for dialectical proverbs was completely explained by differences in the level of FOI.

It is also worth noting that our view of culture as an aggregate of individual differences is well consistent with recent findings on the role of antipathogen psychology in shaping cross-cultural differences. In some regions with a greater prevalence of pathogen, stronger antipathogen psychological responses will be more beneficial than in other regions with a lower prevalence of pathogen. Studies have reported compelling evidence showing that many contemporary cross-cultural differences (e.g., the use of culinary spices, mate preference, family ties, religiosity, ethnocentrism, collectivism-individualism, openness and extraversion, democratization, and conformity) may result, in part, from a conditiondependent psychological adaptation evoked by the local variation of pathogen prevalence (Fincher \& Thornhill 2012; Fincher et al. 2008; Schaller 2011).

In sum, we question Smaldino's argument that culture plays the active role of maintaining and transmitting social organizations of differentiated individuals. By finding other individual 
differences correlated with cultural difference, one can properly eliminate the explanatory power of culture. That is, culture is an aggregate of individual differences in psychological variables within and between groups; it was not designed by group-level selection to maintain the structured organization of individuals. We conclude that Smaldino fails to present the crucial mechanism by which group-level traits are maintained, transmitted, and evolve.

\section{Reinventing the wheel on structuring groups, with an inadequate psychology}

doi:10.1017/S0140525X13002902

\section{Kevin MacDonald \\ Department of Psychology, California State University - Long Beach, Long Beach, CA 90840-0901. \\ Kevin.macdonald@csulb.edu \\ http://www.csulb.edu/ kmacd/paper-Evolpsych.html}

\begin{abstract}
The idea that structured organization of differentiated individuals influences group behavior is not new to evolutionary approaches. An adequate theory of groups needs to incorporate explicit processing, which is central to means-end reasoning involved in leadership and to the construction of ideologies that rationalize group structure. Explicit processing is also central to knowledge of others' reputations, thus enabling altruistic cooperation.
\end{abstract}

Smaldino claims that "The idea that the structured organization of differentiated individuals influences the behavior of human groups is not new" (sect. 8, para. 1) in areas such as cultural anthropology and organizational psychology, but "In discussions of cultural evolution, ... group organization is often ignored" (sect. 8, para. 1). There are certainly examples to the contrary. For example, my work on group evolutionary strategies (MacDonald 1994) discusses several ways that groups are able to structure themselves aimed ultimately at attaining evolutionary goals:

Structuring separation from other groups (e.g., by enforcing endogamy, prohibitions on social intercourse with out-groups, dietary laws).

Structuring within-group altruism and penalizing free riders (e.g., by ostracism of the offending person and the person's family). Smaldino points out that organized groups need not be egalitarian; this is certainly true in the case of traditional Jewish groups. Nevertheless, despite a great deal of differentiation and lack of egalitarianism, there was a strong safety net and pressure on wealthy Jews to contribute to communal charity, and as a rule wealthy Jews patronized other Jews by employing them in their economic enterprises. Economic differentiation within the community was taken for granted, but competition among Jews was closely regulated: Although there was competition among Jews in selling goods within the Jewish community, Jewish communities acted to prevent Jews from, for example, infringing on monopolies held by other Jews in goods and services for non-Jews.

Structuring socialization for in-group loyalty and facilitating the ability to fill particular economic niches (e.g., by supporting high-investment parenting, education, and intellectual ability). Traditional Jewish society was highly competitive, resulting in differentiation among individuals in occupation and social status. For example, men competed to develop a reputation in a socially created reward system in which successful scholars were rewarded with good marriages, business opportunities, and ultimately, higher fertility.

Because he uses an inadequate psychology of groups, Smaldino's explanations for how groups come to be organized fall short. Human groups are able to structure themselves as described above because of explicit processing (MacDonald $2009 ; 2010$ ). Explicit processing is the opposite of implicit (reflexive, modular) processing. It is conscious, controllable, effortful, relatively slow, and involves serial processing of relatively small amounts of information. Explicit processing is involved in creating hypothetical scenarios and in planning for future contingencies (e.g., by groups). As discussed in the rest of the target article, explicit processing enables control of group members, enables ideologies that rationalize group aims and relationships among group members, and enables the ability to track the reputation of group members (e.g., free riders).

Like several other theorists of cultural evolution (e.g., Boyd and Richerson who are cited throughout the target article), Smaldino relies on social learning theory for developing a psychology of cultural evolution and group structuring. The entire emphasis is on cultural transmission, not on how culture is created, for example, by leaders or by discussion among group members. Thus, Smaldino emphasizes leadership as an explanation for group organization but fails to discuss explicit processing necessary for leaders to be able to make plans for the future by using means-end reasoning in the service of attaining ideal goal states (e.g., the creation of blueprints for utopian societies such as Brook Farm in 1840s Massachusetts); there are many important historical groups (e.g., Calvinists) where leaders created ideologies that rationalized group structure (e.g., egalitarian or hierarchical) and group regulation (how to deal with free riders or the less talented) (see MacDonald 2009; 2010).

Explicit processing gives rise to the possibility of characteristically human social controls (e.g., bureaucracies, legal systems) and ideologies (e.g., religions, Marxism) that are able to regulate behavior within a particular historical context. Social controls and supporting ideologies enable group selection between human groups because they are able to enforce discipline within groups, punish defectors, encourage patriotism and obedience to group aims, produce significant levels of altruism, and regulate variation in reproductive success (MacDonald 1994).

Explicitly held beliefs are able to exert a top-down control function over behavior and over evolved predispositions; they are able to exert this control independently of external controls (e.g., punishment) (MacDonald 2008). For example, a person may refrain from engaging in a particular behavior to which he is predisposed as a result of evolved modules (e.g., various forms of aggression [Buss \& Shackelford 1997]), and he may do so because he believes that he would be sent to prison (based on explicit knowledge of the legal system) or because he believes that he will be punished for it in an afterlife (i.e., as a result of a personally held ideology).

Like social controls, the imposition of ideology may be the result of conflict within societies. As in the case of social controls and also because ideologies are so often intricately bound up with social controls, evolutionary theory is unable to predict which ideology will prevail in a particular society. Ideologies may be egalitarian or anti-egalitarian. They may promote the deregulation of human behavior, or they may rationalize strong social controls on behavior.

Explicit processing is also critical for maintaining group structures because it allows explicit appraisals of others' reputations made possible by human language, representational ability, and long-term memory (MacDonald 2008). Indeed, the lack of these abilities in animals goes a long way toward explaining why highly structured groups are a rarity in animals and quite possibly nonexistent. Recent models of cooperation show that altruistic cooperation can evolve if people have access to explicit information on others' histories of interaction in cooperative situations (e.g., Semmann et al. 2005; Smith 2005).

Finally, Smaldino's explanation of group structures in terms of "repeated assembly" is opaque without an illustration from an actually existing human group. Repeated assembly appears to be an attempt to avoid the literature on behavior genetics relevant 
to the division of labor. Smaldino notes that "The repeated assembly view as applied to individual development stands in counterpoint to the view that contributions from genotype ("nature") and environment ("nurture") may be viewed additively or, at best, as interacting in fairly simple ways" (sect. 5.3, para. 1). Thus, Smaldino excludes by fiat the behavior genetic literature on active, passive, and evocative genotype-environment correlations that (in addition to ideologies and social controls as discussed above) would be obvious candidates for developing a theory of division of labor (LaFreniere \& MacDonald 2013; Plomin \& Daniels 1987; Plomin et al. 2008).

\section{Interdisciplinary benefits of a theory of cultural evolution centered at the group-level: The emergence of macro-neuroeconomics and social evolutionary game theory}

\section{doi:10.1017/S0140525X13002914}

\section{Tobias A. Mattei}

Department of Neurological Surgery at Ohio State University, Coordinator of the Interdisciplinary Group for Research in Neuroeconomics, Cognition and Neuroscience, Ohio State University, Columbus, $\mathrm{OH} 43210$.

tobias.mattei@osumc.edu

Abstract: The theoretical concepts developed in the target article, in which the author proposes a new paradigm of cultural evolution based not on the individuals' characteristics, but rather on more global collective properties described as "group-traits" (which emerge when a group of individuals exhibit both differentiation and organization), may have a broader scientific impact that transcend the boundaries of social and evolutionary psychology, paving the way for the emergence of macro-neuroeconomics and social evolutionary game theory.

Most of the major breakthroughs in the history of science occurred not exclusively through pure benchmark research but by the development and application of new theoretical paradigms, which, by challenging long-standing assumptions, completely reformulated the foundational pillars of academic disciplines, providing a new framework through which the experimental data could be understood with basis on a different heuristic perspective. Illustrative examples of such phenomena were the revolution of relativistic mechanics in the early $20^{\text {th }}$ century, which shake the millennial human confidence in the absoluteness of time (Spector 1972), and the emergence of quantum physics, which, by bringing completely new (and sometimes unusual) concepts, such as the wave-particle duality, the discreteness/noncontinuous quality of energy, and the uncertainty principle, challenged some of the most primitive certainties of the human mind regarding the basic nature of the physical world (Greenberger et al. 1999).

In his target article, Smaldino defends a new paradigm of cultural evolution based not on the individuals' characteristics, but rather on more global collective properties described as "group-traits," which emerge when a group of individuals exhibit both differentiation and organization. By providing a broader perspective on the effects of inter-individual interactions in terms of the emergence of new qualities at the group level, the proposed analysis would more powerfully explain the evolution of cultural traits than previous attempts focused exclusively on the properties of the interacting individuals. Besides its more immediate implications to the field of social and evolutionary psychology, such new epistemological approach, as a paradigm that shifts the focus from the individual to the group level as the basic unit involved in the dynamics of cultural evolution, promises to have a major impact over other frontline research areas that, up to now, have operated essentially with basis on the characteristics of few interacting individuals.

For example, although neuroeconomics has emerged as a new research field in which the concepts of economics and probability theory have been used to investigate human decision making under risk and uncertainty (Loewenstein et al. 2008), the vast majority of previous studies in this field have been focused on individual choices and personal intertemporal preferences (Kalenscher et al. 2010; MacKillop et al. 2012) The incorporation of the new concepts developed in the target article, such as collaborative interdependence (instead of cooperation) and levels of selection, may contribute to the enrichment of current investigational protocols, paving the way for the emergence of a macro-neuroeconomics of group decision making and collective behavior. Such a new research field would not only enhance our understanding about the neural basis of the psychological features that are believed to be strongly influenced by social constraints (Mojzisch \& Krug 2008; Sanfey 2007), such as religious and political preferences (both of which have received little attention by past neuroeconomics studies), but would also provide new insights regarding the social evolutionary mechanisms that may cause social virtues (like empathy, altruism, wisdom, social responsibility, and patriotism) (Güroğlu et al. 2009; Meeks \& Jeste 2009) to produce long-term collective effects that transcends their immediate impact in terms of individual inclusive fitness (Moreira et al. 2013).

Similarly, although game theory has flourished in the twentieth century as a new set of powerful research methods dedicated to the analysis of the dynamics of personal choices, mutual cooperation, and conflict under different environmental setups (von Neumann \& Morgenstern 1946), up to now very few game theory studies have employed paradigms that go beyond the level of interaction between few individuals or social entities. In fact, although the effect of natural selection as emerging from repeated interactions between individuals have been incorporated to the general framework of such investigations, giving birth to a new discipline called evolutionary game theory (Maynard Smith 1982), the vast majority of studies in this field have been devoted to the analysis of population dynamics of biological ecosystems (Hofbauer \& Sigmund 1998; Zeeman 1980). In a critical analysis, the major obstacle to extending game theory methodology to more complex social situations is that, unlike the simple interactions observed in natural systems (such as the predator-prey or host-parasite relations), human social systems involve a much more complex network of hierarchical social structures interacting through heterogeneous, interdependent, and dynamic relationships that often challenge the ability of mathematical modeling.

Additionally, the unique capacity of human beings of predicting other individuals' behavior with basis on common knowledge assumptions (the so-called theory of mind) (Saxe \& Kanwisher 2003), as well as to rationally analyze the dynamics of their surrounding environment and to adapt their personal behavior accordingly, poses another level of complexity in which not only actual facts, but also the subjective perception of such facts plays a decisive role in final decisions and observed collective behavior. These are some of the reasons why previous experimental research in behavioral and social sciences have yielded results that are very different than those predicted by standard models of game theory involving players who employ strict instrumental rationality to maximize their expected utility (Colman 2003; Gopher et al. 2000).

In this context some important aspects highlighted by Smaldino regarding the emergence, maintenance, and adaptiveness of group-level traits (such as the key role of labor division, leaders and religion, the importance of repeated assemblies, and the influence of technology) may provide the basic elements for construction of more complex models of social interaction. It is important to emphasize that such type of theoretical research have not only important consequences for future academic investigations, but also very practical implications, as exemplified by the emergence of applied disciplines with major social applications such as neuromarketing (Fisher et al. 2010) and neurolaw (Meynen 2013).

In summary, although the main point defended in the target article was the adoption of group-level traits in the analysis of cultural evolution, the complex theoretical pathway through which Smaldino struggled to safeguard his thesis constitutes a legacy 
Commentary/Smaldino: The cultural evolution of emergent group-level traits

of new conceptual developments that is expected to strongly influence the future of basic science and applied research in several different fields. As Ernest Hemingway said:

It is good to have an end to journey toward; but it is the journey that matters, in the end.

\section{Collaboration in classical political economy and noncooperative game theory}

\author{
doi:10.1017/S0140525X13002926
}

\section{Roger A. McCain}

Department of Economics and International Business, Lebow College of Business, Drexel University, Philadelphia, PA 19104.

mccainra@drexel.edu http://www.drexel.edu/

Abstract: This commentary suggests (1) that there are precedents for Smaldino's "collaboration" in the history of economic thought before 1900 and (2) that the distinction of collaboration from what is thought of as cooperation in game theory is less clear than Smaldino suggests.

Smaldino's reconsideration of the importance of group traits that involve differentiation of roles is useful and important. However, it could be enriched by a more complete understanding of game theory and also by a revisit to some of the ideas of the classical political economists. This comment will take the two points in reverse order.

We find a parallel in Adam Smith's Wealth of Nations (Smith 1994), in which he discusses the division of labor and illustrates it with the famous example of the pin factory, among others. According to Smith, in the first sentence of Chapter One, "The greatest improvement in the productive powers of labour, and the greater part of the skill, dexterity, and judgment with which it is any where directed, or applied, seem to have been the effects of the division of labour."

In his Principles of Political Economy, John Stuart Mill (1987) returned to Smith's discussion of the division of labor. Mill clearly thought of the division of labor as a (if not the) fundamental cause of improving standards of living. Mill used the term "complex co-operation" to designate what Smith had called "division of labor," and he distinguished it from "simple cooperation," which seems to correspond to what Smaldino (sect. 2.2, para. 2), following Wimsatt (1974), calls "aggregate" group properties (or group actions). Mill stresses that complex cooperation ("collaboration" for Smaldino) offers further increases in labor productivity beyond that available through simple cooperation.

In McCain (2014, Ch. 2) the theory of production shared by Smith and Mill is called a "complex combination of labor" theory, that is, one that holds that labor productivity is primarily determined by the complex combination of labor, rather than by the combination of simple labor with great quantities of other resources. Another example of a complex combination of labor theory of productivity is found in the writings of the Elder Austrian School, (esp. Menger 1871/1976), which stresses the complexity of production in terms of the tools used. These two strains of complex combination of labor theory were synthesized by Ely (1901) but largely lost sight of in the twentieth century.

Complex cooperation in the writings of Mill seems to correspond more closely to what Smaldino calls collaboration than to what he calls cooperation. No doubt this terminological novelty is worthwhile: the word "cooperation" has a great deal of baggage. It is not so much ambiguous as polyguous.

Nevertheless, Smaldino's understanding of game theory and its relation to cooperation or collaboration seems incomplete. Smaldino stresses some rather simple game examples, but these by no means exhaust the resources of noncooperative game theory. Here is an example, only slightly more complex, that seems to capture both complex cooperation as understood by Mill and collaboration as understood by Smaldino (esp. sect. 2, para. 1; sect. 4.1, para. 1).
Table 1 (McCain). A Smith-Mill Game

\begin{tabular}{|c|l|l|l|l|}
\hline \multirow{2}{*}{$\begin{array}{c}\text { First payoff to Worker } 1 ; \\
\begin{array}{c}\text { Second payoff to } \\
\text { Worker } 2 .\end{array}\end{array}$} & \multicolumn{3}{|c|}{ Worker 2 } \\
\cline { 2 - 5 } & Work alone & Task 1 & Task 2 \\
\hline Worker 1 & Work alone & 1,1 & 1,0 & 1,0 \\
\cline { 2 - 5 } & Task 1 & 0,1 & 0,0 & 4,4 \\
\cline { 2 - 5 } & Task 2 & 0,1 & 3,3 & 0,0 \\
\hline
\end{tabular}

The Smith-Mill game shown in Table 1 is adapted from McCain (2014), Chapter 3. Essentially the game in McCain's Chapter 3 expands the "Stag Hunt" game to allow for division of labor. The game at Table 1 modifies it further to allow for different aptitudes along the lines of Mill's discussion of complex division of labor and Smaldino's kayak maker and seal hunter example (sect. 4.1, para. 3). The players are Worker 1 and Worker 2. The strategies are to work alone or work collaboratively taking Task 1 or Task 2 . Assume Worker 1 has a "knack" for Task 1 and Worker 2 for Task 2. To realize the benefits of collaboration, it is necessary that each worker take one of the two tasks.

This game has three distinct Nash equilibria that are ranked in Paretian terms. As such, it mixes elements of a coordination game (e.g., the Stag Hunt) and an anticoordination game (Tardos \& Vazirani 2007). One possible solution is a hierarchy, whereby one of the two is designated as "leader" and the other as "follower." The "leader" directs the strategies of both. In this case, it does not matter which player is designated as "leader," because it is in the interest of both to choose Task 1, Task 2. (This is further discussed in McCain 2014, Ch. 7, sect. c).

It seems that the Nash equilibrium in the rightmost column in the second row from the bottom is the collaborative outcome of this game as Smaldino understands it. It is also the unique cooperative solution. For noncooperative games in standard form (such as Table 1), there seems to be no very general way of identifying a cooperative solution. In this case, however, we can rely on Aumann's (1959) criterion as the distinct cooperative solution to the game. The collaborative solution in the rightmost column is the only strong Nash equilibrium in the game. It will also correspond to each of the several criteria for solutions of cooperative games.

Responding to social-dilemma examples along the lines of public goods, Smaldino writes, "Yet, the group-level behavior is defined not simply in terms of individuals donating or withholding contributions, but in terms of each individual doing his own part in a coordinated and organized manner. These hunters are doing more than cooperating: they are collaborating" (sect. 4.1, para. 1). Nevertheless, they are enacting the cooperative solution, not to a simplified game, but to the game they are playing.

\section{Individual-level psychology and group-level traits}

doi:10.1017/S0140525X13002938

Michael Muthukrishna and Mark Schaller

Department of Psychology, University of British Columbia, Vancouver, BC V6T 1Z4, Canada.

michael@psych.ubc.ca schaller@psych.ubc.ca

http://www2.psych.ubc.ca/ schaller/schaller.htm

Abstract: Psychological research on social influence illuminates many mechanisms through which role differentiation and collaborative interdependence may affect cultural evolution. We focus here on psychological processes that produce specific patterns of asymmetric influence, which in turn can have predictable consequences for the emergence and transmission of group-level traits. 
Smaldino makes a compelling case that role differentiation, collaborative interdependence, and emergent group-level traits play an important role in cultural evolution. Smaldino offers preliminary speculations about processes through which group-level traits might emerge, but a lot remains to be specified. What specific role differentiations have implications for the emergence of important group-level traits? What specific proximal mechanisms might account for the emergence of these group-level traits and for their change over time? How might these processes be affected by specific circumstances? Answers to these questions require input from the sciences that focus on proximal mechanisms.

It may be especially useful to draw upon insights from the psychological sciences-especially research that explores the many ways that individuals influence each other during interpersonal interactions. Particularly relevant are lines of research documenting specific ways that influence outcomes differ depending on the social context-including the roles occupied by the individuals involved (Cialdini \& Goldstein 2004; Hogg 2010; Wood 2000).

It is easy to overlook this literature when addressing questions about long-term cultural outcomes, because psychological inquiry focuses on the immediate actions and outcomes of individuals and rarely considers their population-level consequences. But there are exceptions to this disciplinary restraint (Resnick et al. 1991; Schaller \& Crandall 2003).

Consider, for example, research on dynamic social impact theory (DSIT; Harton \& Bullock 2007). Drawing upon a few basic principles of social geography, social interaction, and social influence, DSIT shows how mutual influence that occurs during dyadic interactions has, over time, inevitable consequences for population-level outcomes. These emergent outcomes include changes in the popularity of beliefs and behaviors and changes in the extent to which these beliefs and behaviors correlate and cluster. Furthermore, DSIT shows how the psychology of social influence can create and sustain patterns of diversity within a cultural population. Diversity - of beliefs, behaviors, aptitudes, etc. is an outcome of particular relevance here. As Smaldino observes, diversity sets the stage for collaboration, which can then give rise to new group-level traits. Second, because diversity is the fuel that fires the engine of evolution, it has implications for cultural evolution more generally.

One important reason why the psychology of social influence produces predictable population-level consequences is because, within any social interaction, influence is rarely symmetrical. Some individuals are more influential; some individuals are more influence-able. DSIT typically treats these influence asymmetries as random variation. But they are not just random. The psychological literature documents specific kinds of role differentiations that have specific implications for patterns of asymmetric influence; this, in turn, can have predictable long-term consequences for cultural diversity and other group-level traits.

Leadership roles are one obvious example. Smaldino speculates that leadership, and its consequences, is one likely means through which group-level traits emerge. There is an extensive empirical literature on the psychology of leadership and followership and implications for group outcomes (Van Vugt et al. 2008). By applying insights from this literature, it will be possible to predict group-level traits with greater precision.

Other forms of asymmetric influence arise from a variety of other social distinctions-some obvious and some not-that connote differences in power, dominance, expertise, or prestige. These differences have consequences for individual-level cognition and additional consequences for social influence (Cheng et al. 2013; Fiske 2010; Galinsky et al. 2008). Because of these asymmetrical influence implications, there will be further consequences for the emergence and transmission of group-level traits.

It may also be productive to explore the implications of sex differences. The male/female distinction is perhaps the most fundamental form of collaborative interdependence within the human species (in the sense that men and women collaborate interdependently to produce offspring). The mating game is a dynamically unfolding process in which individuals' thoughts and actions are influenced by the presumed thoughts and actions of other men and women in the immediate vicinity. But men and women do not influence each other in exactly the same way; their influence is predictably asymmetrical. The implication, explored in research on "dynamical evolutionary psychology," is that the specific distributions of men and women within a population, and the specific characteristics of those men and women (e.g., the extent to which they are available or unavailable as mates), can affect the emergent properties of the entire population (Kenrick et al. 2003). The implications of sex differences for group-level traits are not limited to the domain of mating behavior. The male/female distinction has profound implications for division of labor and distribution of knowledge across a wide range of behavioral domains (Fried 1967; Wood \& Eagly 2010). Research that systematically integrates the psychological literatures on sex differences (Geary 2010) and social interaction with Smaldino's perspective on group-level traits is likely to reveal additional novel implications for cultural evolution.

The psychological processes that govern social interaction and social influence - and lead to emergent group-level traits are themselves moderated by additional features of local ecologies. For example: the prevalence of infectious diseases in the local ecology appears to have many relevant implications. Among other outcomes, the sociological and psychological bases of asymmetric influence - rigid status hierarchies, authoritarian attitudes, etc. - are more evident under circumstances of higher disease prevalence (Murray et al. 2011; 2013). The implication is that when diseases pose less of a threat, a more diverse set of beliefs and behaviors are likely to be expressed and maintained within a population. So, by carefully considering the specific psychological processes that govern the emergence of group-level traits, we may also be able to more fully identify connections between ecological circumstances and cultural evolution.

The take-home message is this: to realize the vast potential of the perspective outlined by Smaldino, it will be helpful to draw more fully on the vast psychological literature on social influence. As a happy corollary, this kind of conceptual integration will benefit the psychological literature too.

\section{Cultural evolution and emergent group-level traits through social heterosis}

\author{
doi:10.1017/S0140525X1300294X
}

Peter Nonacs ${ }^{\mathrm{a}}$ and Karen M. Kapheim ${ }^{\mathrm{b}}$

${ }^{a}$ Department of Ecology and Evolutionary Biology, University of California, Los Angeles, CA 90095; ' ${ }^{\mathrm{b}}$ Department of Entomology, Institute for Genomic

Biology, University of Illinois at Urbana-Champaign, Urbana, IL 61801. pnonacs@biology.ucla.edu

https://www.eeb.ucla.edu/index.php

kapheimk@illinois.edu

http://www.igb.illinois.edu/

Abstract: Smaldino proposes emergent properties of human groups, arising when individuals display both differentiation and organization, constitute a novel unit of cultural selection not addressed by current evolutionary theory. We propose existing theoretical frameworks for maintenance of genetic diversity - social heterosis and social genomes can similarly explain the appearance and maintenance of human cultural diversity (i.e., group-level traits) and collaborative interdependence.

Human groups are not aggregates of interchangeable parts. The "who" of groups matters. Diversity across individuals produces collective group phenotypes that are both variable across groups 
and impossible for any single individual to express. Smaldino insightfully argues that such emergent group-level phenomena (i.e., collaborative interdependence) drive human cultural evolution. He further proposes these units of selection are inadequately represented in current models of cultural multilevel selection (cMLS) that follow an inclusive fitness approach. We submit, however, that an MLS model for group-emergent traits well ensconced in inclusive fitness reasoning already exists. This is social heterosis - where group diversity, itself, can be the mutually advantageous trait (Nonacs \& Kapheim 2007). Under certain conditions, individuals in heterogeneous groups achieve higher fitness than they would in homogeneous groups, and diverse groups experience more beneficial collective properties than uniform ones do. Social heterosis models examine emergent group-level fitness through nonadditive benefits that individuals gain from being in specific differentiated and organized units. Although originally applied to the maintenance of genetic diversity, social heterosis is also relevant to cultural evolution. The possibility that groups can have collaborative interdependence is an intrinsic part of social heterosis.

Collaborative interdependence can arise within social heterosis theory through social genomes. A social genome forms across group members that possess complementary alleles at key genes (i.e., fitness epistasis across individuals). For example, Nonacs and Kapheim (2012) accurately modeled the evolutionary progression of HIV to AIDS within human hosts. As Smaldino describes for collaborative interdependence, replicative fitness of HIV increased nonadditively when genetically different clones evolved complementary capabilities and could co-infect the same cell. In other words, once an HIV social genome evolved, immune system collapse followed. Just as the social genome is an example of intergenomic epistasis, collaborative interdependence is an example of cultural epistasis. Cultural epistasis may indeed provide the emergent fitness properties that help maintain cultural diversity.

Culturally based social genomes could arise in multiple contexts in humans, and as Smaldino persuasively argues, perhaps too much weight has been given to overt cooperation, with its implicit assumptions of altruistic or self-sacrificial behavior. Social heterosis can arise without explicitly cooperative interactions or any direct interactions at all. For example, consider Smaldino's collaboratively interdependent "seal-hunters" and "kayak-builders." Social heterosis is evident as cultural epistasis: For example, successful groups require both skills to be present and payoffs are nonadditive. If hunting is always more prestigious with higher payoff, then the ratio of hunters to builders will increase in all villages, as hunters can support more children (if hunting is genetically heritable) or more children will decide to hunt (as a cultural preference from observing outcomes). Villages with too many hunters, however, will flounder as a result of few and poor boats, leading to fewer offspring for repopulating and export. In contrast, villages with healthier mixes of occupations will export more new hunters and builders. In general models of such scenarios (Nonacs \& Kapheim 2007; 2008), population trait diversity can be maintained if the mean fitness of traits disadvantaged within groups exceeds the fitness of advantaged traits when they are in less diverse groups (i.e., builders in diverse villages do better than hunters in less diverse villages, although within any given village, hunters always do better). Therefore, between-group variance across village cultural genomes would maintain skill set diversity in populations without requiring either kin-biased nepotism or reciprocated cooperation.

Smaldino proposes novel mechanisms are needed to explain human group-level traits. However, the three proximate mechanisms Smaldino proposes for the emergence of group-level traits are encompassed in the emergence and maintenance of a social genome through social heterosis. For the HIV example, leadership arises because epistasis across loci appears to evolve in a predictable order, division of labor arises when complementary alleles give rise to new function, and repeated assembly occurs via cell death and infection. Nonadditive benefits of intergenomic epistasis enhance the probability that group-level properties will be preserved across repeated assemblies. Analogously, collaborative interdependence leads to and maintains emergent properties of cultural groups.

Social heterosis and social genomes are MLS models and fit comfortably in an inclusive fitness framework such as Hamilton's Rule (i.e., traits are selectively favored when benefits provided to relatives exceed costs to the actors, or $r b-c>0$ ). The key to social heterosis is that relatedness, benefit, and cost are not independent variables; instead, benefits provided $(b)$ or costs incurred $(c)$ correlate with relatedness $(r)$ at the group level. For example, Smaldino highlights the role high genetic relatedness plays in social insect evolution. Although the initial evolution of cooperative breeding and specialized worker castes correlate with high relatedness through strict monogamy (Boomsma 2013), subsequent evolution has often led to low relatedness, as polygamy has independently appeared in more than 20 different taxonomic lineages (Hughes et al. 2008). Genetic diversity and "who" resides in colonies significantly affects colony-level fitness (Wray et al. 2011), such that higher $b$ and lower $c$ associate with lower $r$. Close relatedness is disadvantageous when group diversity benefits are positive and nonadditive. Indeed, diversity-producing social genomes of low relatedness may be the hallmark of behavioral complexity and ecological success throughout the social Hymenoptera (Nonacs 2011a; 2011b).

In conclusion, social heterosis and social genomes models can predict the evolution of genetic traits, and we propose they similarly apply to cultural ones. It does not matter if "kayak-building" is cultural rather than genetic because the trait is still transmitted with fidelity. Unlike genes, culture transmits both vertically and horizontally across individuals and can be malleable within individuals over their lifetimes. Hence, cultural traits increase in frequency through differential net rates of phenotypic conversion within populations. Just as the logic of natural selection applies to how gene frequencies change across generations, the same logic can apply to the spread and evolution of human culture, behavior, and practices. Our rejoinder to Smaldino's view that, "models are needed that capture the difference between the social spreading of a particular individual-level trait and the emergence of group-level behaviors that rely on differentiation and organization" (sect. 8, para. 2), is that they already exist - we just need to use them.

\section{Homogeneity of mind can yield heterogeneity in behavior producing emergent collaboration in groups}

\author{
doi:10.1017/S0140525X13002951
}

\section{Rick O'Gorman}

Department of Psychology, University of Essex, Colchester CO4 3SQ, UK. rogorman@alumni.binghamton.edu http://www.essex.ac.uk/psychology/department/people/ogorman.html

Abstract: The evolved psychological process for producing social norms is both needed to facilitate emergent group-level traits and capable of delivering such a process. I discuss how this process can work to generate group-level traits and how specific mechanisms established to buttress social norms similarly can explain how group-level traits are supported.

Smaldino rightly critiques cultural evolutionary approaches for falling short of providing models to fully understand human cultural behavior. In particular, he focuses on the failure of these cultural evolutionary approaches to address emergent group-level traits, which Smaldino defines as collaborations that incorporate role 
differentiation and organizational complexity. Existing cultural evolutionary approaches focus on models of culture that rely on individual-level mechanisms that yield fitness benefits to individuals; insofar as there is a group benefit attributable to a cultural trait in these models, it is a result of the transmitted information happening to produce a group-wide benefit; the underlying traits remain individually selected. As such, these models do not readily address the complex cooperation that we rightly view as a benefit of the cultural process and which offer group-level benefits. Moreover, the handful of postulated cultural learning traits of the cultural evolutionary frameworks proposed to facilitate cultural learning processes are content-independent; there is little in the cultural evolution models that can account for how psychologically we can process a suite of cultural behaviors that function in an integrated fashion.

However, although cultural evolutionary approaches have failed to consider such psychologically sophisticated processes, Smaldino wrongly suggests that this problem has been previously ignored by the literature. The notion of complex forms of cooperation has been discussed, and not just for humans. Such group-level traits drawing on differentiated roles occur in the behavioral suites of non-human animals, particularly social predators, although these behaviors are not usually thought to be cultural (though there is likely to be some learning, part individual, part socially facilitated). Drawing on Tomasello and colleagues (2005), Smaldino incorrectly suggests that collective hunting behavior in chimpanzees constitutes nothing more than each individual doing their own thing, just in concert. In fact, the hunting techniques of chimpanzees, as well as lions and wolves, show that there is clear role differentiation that can be critical to a successful hunt, with individuals adopting key roles to drive the prey into traps, corralled by other individuals, and the kill made by another.

In humans, group-level adaptations that entail distinct roles have been previously explored (O'Gorman et al. 2008a). For example, Hutchins (1995a; 1995b) has examined a number of social situations that require close collaboration between team members, analogous to the egg hunters discussed by Smaldino. Hutchins examines cockpit crews working to ensure the safe landing of airplanes, and the crew of a ship in bringing it safely into harbor. Closer to Smaldino's concerns, Wilson (2002) similarly discusses group-adaptive traits that are highly cultural, such as the water temples of Bali, in the context of cultural adaptations. Smaldino himself identifies norm-enforcing institutions as a means to transmit group-adaptive traits.

The question, of course, is: How can these group-adaptive traits operate through the cultural process? In that sense, a key focus for advances in this area should be to draw from our understanding of social norms. Although often thought of as a behavioral conformity process, norms can be expanded to any cognitive variant that can be socially shared. For example, belief in the Christian god in the medieval cultures of Europe was a norm as much as any religious ritual. Given that humans are equipped to share intentionality (Tomasello et al. 2005), conformity around beliefs, goals, intentions, and so on readily come under the remit of our normative psychology. Research suggests that humans are particularly evolved to respond to social norms. Alongside the selfevident conformity that is so evident in human behavior, O'Gorman et al. (2008b) have shown that humans have an enhanced ability to retain knowledge of normative information. Adoption of shared goals and intentions in turn can facilitate individuals engaging in different but coordinated behaviors that target the normative goals. Of course, such a process requires that individuals adopt the appropriate behaviors to achieve the goals, the learning of which may occur implicitly or explicitly.

As long as there are behavioral correlates to the cognitive norms, individuals and groups can regulate the functioning of their members, something that is necessary for any costly norm. Just as the belief in the Christian god yielded concomitant socially visible behaviors (attendance at church services, gestures of respect to religious icons, and so on), so any group goal that has an impact in the actual world will have visible markers of conformity to the shared goals. Individuals who are not readily cooperating will be identifiable and can be brought into line through various evolved mechanisms that prevent free riding on norms (O'Gorman et al. 2008b; Wilson \& O'Gorman 2003), including gossip (Ellickson 2009), ostracism (Spoor \& Williams 2007), and punishment (O'Gorman et al. 2009).

To that end, once specific cognitive norms are established and accepted within a group, subservience to such norms will readily follow, just as subservience to behavioral norms tends to follow. As such, it is worth noting that recognition that the normative process is fundamental to group-level traits means that cooperative dispositions once again are restored to central importance, even for Smaldino's emergent group-adaptive traits. Without cooperation in the form of conformity to costly social norms, there can be no emergent group-level traits.

Smaldino has succeeded in focusing our attention on an important problem for cultural models of human cooperation: Although much of culture may operate in a near-unconscious manner, there are important components where individuals need to be cognizant of particular suites of complex collective behaviors - group-level traits - where members must operate in line with what is known to be necessary to succeed in such behaviors. This is not to say that group members will be cognizant of all the behaviors involved in successful completion of such behaviors, but they will need to be aware of shared goals and likely will be highly sensitive to evidence that group members are not contributing appropriately to those goals.

\section{Emergent group traits, reproduction, and levels of selection}

doi:10.1017/S0140525X13002963

\section{Samir Okasha}

Department of Philosophy, University of Bristol, Bristol BS8 1TB, UK. Samir.Okasha@bristol.ac.uk http://www.bristol.ac.uk/school-of-arts/people/samir-okasha/index.htm

Abstract: All group traits, "emergent" or otherwise, are ultimately dependent on the traits and behaviours of the individuals that constitute the group. Unless a process of "group reproduction" is envisaged, this means that the evolution of group traits can in principle be studied in an individualistic way, by studying the dynamics of the underlying individual traits on which they depend.

There is much that I applaud in Smaldino's article, in particular his emphasis on the importance of division-of-labour, role differentiation, and group-level functional organization in explaining the success of human (and other) groups. These features have of course been noted before, but I agree with Smaldino that they have been somewhat neglected by traditional models of social evolution (both genetic and cultural), which tend to focus on the conditions needed for an altruistic trait to spread in a group-structured population. These models are clearly important, but I share Smaldino's sense that they are not the whole story.

A theme that runs through Smaldino's article is the inadequacy of what might be called an "individualistic" or bottom-up approach to understanding the evolution of emergent grouplevel traits. Smaldino argues that such traits can only be expressed by groups, are not mere aggregations of individual-level traits, and evolve by a multilevel selection process that cannot be reduced to or redescribed as individual selection in a structured population. A number of points about this argument merit discussion.

Firstly, I agree that it is intuitive to regard some group-level traits, in humans and other species, as "emergent." However, the emergent/aggregate trait distinction is notoriously difficult to make 
Commentary/Smaldino: The cultural evolution of emergent group-level traits

precise (cf. Okasha 2006). After all, any group trait is presumably ultimately dependent on the traits and behaviours of the constituent individuals in the group, in the sense that once the individuals' traits are fixed, this then fixes the group trait. (Philosophers call this a relationship of "part-whole supervenience.") In cases where the pattern of dependence is particularly complicated, or where the underlying individual traits are heterogeneous, it may be natural to think of the group trait as "emerging" from them, but this is inevitably a matter of degree; thus, "emergent traits" do not constitute a sharply defined category.

Secondly, the question of how best to understand the evolution of a group trait, be it emergent or not, depends heavily on how the group trait gets transmitted down the generations. This in turn depends on whether reproduction occurs at the individual or the group level. In a human group, however functionally organized it is, the constituent individuals all retain reproductive capacities; so presumably, the group trait gets transmitted via individuals transmitting, to their descendants, the individual-level traits on which the group-level trait supervenes. ("Descendants" can be understood to include cultural, as well as genetic, descendants.) I say "presumably" because in principle, one could imagine a process of "group-level reproduction" in which some groups beget other groups, for example, by fission, and directly transmit the group trait to their (group) offspring. However, such a process seems rather unlikely in the case of human groups, and Smaldino does not appear to envisage it.

If it is right that the group traits in which Smaldino is interested are ultimately dependent on individual traits, and if no process of "group-level reproduction," decoupled from individual-level reproduction, is being envisaged, then in principle it must be possible to understand the evolution of the group trait in an individualistic way, by tracking the evolutionary dynamics of the underlying individual trait(s) on which the group trait depends. Thus, I am unconvinced by Smaldino's argument that an individualistic approach is in principle impossible.

Thirdly, I do not entirely agree with Smaldino that extant theories of multilevel selection and kin selection are silent about emergent group traits. The modelling approach known as "contextual analysis," for example, introduced by Heisler and Damuth (1987), explicitly treats an individual's fitness as a function of its own traits and the traits of the group to which it belongs, which can include "emergent" traits that are not mere aggregations of individual-level traits (cf. Frank 2013; Okasha 2006). However, Smaldino is right that the majority of models of multilevel selection do not explicitly incorporate emergent group-level traits into their formal analyses; the "group traits" that feature in such models are typically the frequencies of individual traits (or genes) within the group. But because all group traits, emergent or not, must ultimately depend on individual traits, one cannot assume that such models are incapable of shedding light on the evolution of the group traits that interest Smaldino.

Finally and relatedly, Smaldino argues against the widespread view that multilevel selection and inclusive fitness (or kin selection) models are essentially equivalent because the evolution of any (individual) social trait or gene can be expressed using either. Smaldino argues that this equivalence breaks down when a "between-levels" perspective is adopted, which takes suitable account of emergent traits. I remain unconvinced by this for the reasons given above, but I agree with Smaldino that social evolution theorists have been too quick to endorse the equivalence of multilevel and kin selection. Although it is true that the two are formally or predictively equivalent, in that both yield identical conditions for an allele to increase in frequency, it does not follow that they are causally equivalent. In forthcoming work, I argue that in some cases, multilevel selection yields a better causal representation of social evolution, which in other cases kin selection does better (Okasha, in press).

These critical points notwithstanding, Smaldino's article offers many valuable insights into what an improved theory of human cultural evolution might look like.

\section{Explaining group-level traits requires distinguishing process from product}

\author{
doi:10.1017/S0140525X13002975
}

\section{Karthik Panchanathan, ${ }^{a}$ Sarah Mathew, ${ }^{\mathrm{b}}$ and Charles Perreault ${ }^{\mathrm{b}}$}

a Department of Anthropology, University of Missouri, Columbia, MO 65211; ${ }^{\mathrm{b}}$ School of Human Evolution and Social Change, Arizona State University, Tempe, AZ 85287.

karthikpanchanathan@gmail.com

http://www.missouri.edu

Sarah.Mathew@asu.eduｃharles.perreault@asu.edu

http://www.asu.edu/

Abstract: Smaldino is right to argue that we need a richer theory of grouplevel traits. He is wrong, however, in limiting group-level traits to units of cultural selection, which require explanations based on group selection. Traits are best understood when explanations focus on both process (i.e., selection) and product (i.e., adaptation). This approach can distinguish group-level traits that arise through within-group processes from those that arise through between-group processes.

We agree with Smaldino's thesis: many important aspects of human behavior are best described as group-level traits, emergent properties of social groups characterized by differentiation and organization. Crowdsourcing provides a vivid and contemporary illustration. Wikipedia is the product of an immense collaboration of specialists organized into different roles like author and editor. Such a work cannot be expressed by or reduced to any one of its millions of contributors. How should we make sense of group-level properties like this? According to Smaldino, theory leans too heavily on the $\mathrm{N}$-person prisoner's dilemma model in which groups do best when everyone contributes but individuals do best by withholding contributions. This model forces us to think about social groups and behavior in terms like "cooperation," "freeriding," and "altruism." Many group-level properties, especially those with emergent, rather than additive properties, do not fit into this framework.

Although sympathetic to Smaldino's call for a richer theory of group-level traits, we disagree that group-level traits necessarily constitute "a unit of cultural selection that is not encompassed by selection on individuals" (sect. 1, para. 2). This assertion seems to yoke together group-level traits and group-level selection. As we will argue, this need not be the case. The deeper problem is that Smaldino does not clearly distinguish selection from adaptation. Making sense of phenotypic evolution (including group-level traits) is best done by focusing on both process (i.e., building models of selection to determine why one phenotype results rather than another) and product (i.e., studying adaptations to infer the historical selective pressures). Focusing on group-level traits without explicitly modeling selection processes results in a phenomenological approach that obscures and confuses alternative mechanistic explanations. Smaldino gets caught in this trap when discussing caste differentiation in eusocial insects: "the trait [caste differentiation and social organization] does not emerge from the collection of individuals possessing those properties, but is merely a statistical description of their organization" (sect. 3, para. 5). It is not obvious why a Roman Legion (discussed in sect. 2) represents a group-level trait, while an insect colony does not. What would help is an understanding of both the consequences of the traits and how they evolve.

To illustrate our point, let us imagine a group of individuals stranded on an island, isolated from the rest of the world. For the sake of argument, we endow these individuals with the capacity for division of labor and trade (as Smaldino notes, a crucial ingredient in the emergence of group-level traits). Capacities like these emerge over evolutionary timescales. Because we are interested in the emergence of group-level traits over historical timescales, we can take these capacities as given. Though capable of division of labor, our hypothetical islanders do not practice it. Instead, they embody Marx's 
manifesto: no one is a specialist; everyone is a generalist. During the day, all are fisherman; at night, all philosophers.

Suppose now that some individuals specialize, exclusively fishing or philosophizing. With increasing returns to specialization, these innovators produce more fish or more knowledge than their comrades. If the islanders value leisure-and who does not? - they can have more of it by trading with each other. Fishers trade their surplus catch to philosophers in exchange for knowledge (or whatever it is that philosophers produce). And both parties enjoy more leisure. Specialization-and-trade will quickly spread throughout the community. The benefit of division of labor has transformed our society into one that is differentiated. An outside observer might argue that the economy should be understood as an emergent, group-level trait. But what process caused this trait? Surely not group selection, as there were no competing groups. Instead, the emergent, group-level trait resulted from within-group forces in which individuals made choices to maximize utility.

The observer might then object that this hypothetical fishingand-philosophizing economy does not, in fact, constitute a group-level trait. After all, the products of labor flow to individuals; they are not properties of groups. Fair enough. Suppose now that fishermen discover that they can catch more fish by working together in collaborative teams, complete with differentiated roles and social organization. (As with division of labor, we are assuming the capacity for collaboration, not explaining it.) These groups fish together for some time, reap profits that are then split among group members, and then individuals go their separate ways. Smaldino argues that ephemeral co-ops like this constitute trait groups but are not "consistent enough over time to constitute a unit of selection" (sect. 3, para. 5). Maybe so. But in our example, the group-level trait results from individuals maximizing utility, not group selection.

Finally, let us suppose that the island is bridged to other islands. People are free to move to whichever island they so desire. On some islands, philosophizing is an individual affair, and so knowledge systems are limited. On other islands, philosophers long ago founded academies in which knowledge could be shared and therefore grows further and faster. Islands with academies are far more attractive to immigrants than islands without them. When migrants vote with their feet, group-level traits can spread through a group-level process (Boyd \& Richerson 2009b). In addition, philosophically starved islanders might emulate their more successful neighbors by founding academies, representing another form of group selection (Boyd \& Richerson 2002). In both cases, a group-level trait spreads because of grouplevel selection.

Although we agree with Smaldino that more attention must be paid to group-level traits, we want to stress that this focus on adaptation must be combined with a focus on selection. Group-level traits, as defined by Smaldino, may arise through group selection, but they may also arise through within-group processes. When multiple processes operate simultaneously, it is all the more crucial to understand how they interact in generating adaptation.

\section{The substance of cultural evolution: Culturally framed systems of social organization}

\section{doi:10.1017/S0140525X13002987}

\section{Dwight W. Read}

Department of Anthropology and Department of Statistics, University of California-Los Angeles, Los Angeles, CA 90094.

dread@anthro.ucla.edu

http://www.ucla.edu/

Abstract: Models of cultural evolution need to address not only the organizational aspects of human societies, but also the complexity and structure of cultural idea systems that frame their systems of organization. These cultural idea systems determine a framework within which behaviors take place and provide mutually understood meanings for behavior from the perspective of both agent and recipient that are critical for the coherence of human systems of social organization.

Smaldino advances an argument similar to that of Lane et al. (2009) regarding the need to make "a shift in perspective, from population thinking to organization thinking" (2009, p.12, emphasis in the original) by arguing that models of cultural evolution have not taken into account contextualization of human behavior through systems of organization that make human behavior more complex than just as epiphenomena of individual level traits. This leads Smaldino to consider three levels for modeling selection acting on traits: (1) individual traits, (2) multilevel traits (traits aggregated over behaviors engaged in collectively by interacting group members), and (3) group traits expressed through the institutionalized organization of role-differentiated individuals (sect. 1, para. 3). Group traits are, in Smaldino's view, distinguishable by making use of the "specific organization of [role] differentiated individuals" (sect. 2.2, para. 2), with selection acting on systems of organization that maintain internal differentiation of individuals, hence acting on emergent group behavior (sect. 2, para. 5) rather than on individual behavior expressed collectively, as is the case for multilevel selection.

Although valid questions can be raised about Smaldino's characterization and differentiation of these three different levels, especially with regard to his thesis that group success in human societies largely comes from "the organization of a welldefined collection of differentiated individuals all participating in a group-level behavior" (sect. 3, para. 4), my focus here is on the phylogenetic trend going from solitary to structured groups and from individual to emergent to culturally framed behavior as we evolutionarily move toward our species, Homo sapiens, with its subdivision into highly differentiated societies. The picture drawn by Smaldino, using his wording for the limitations of multilevel selection, "is not incorrect, but it is incomplete" (sect. 3, para. 4).

The evolution of human social systems centers around the development of systems of organization that incorporate, rather than suppress, individual differentiation (Read 2012). Briefly, the phylogenetic trend toward increased individualization of behaviors that we see when we traverse the primates toward Homo sapiens is paralleled by social complexity increasing exponentially with the number of individualistic group members (Read 2012, Fig. 4.3). This increase was accommodated not only through neurological changes (Dunbar 1998), but by changes in the structural organization of social units that culminated, from a biological perspective, in reduction of the size of chimpanzee social units (Read 2012)-where chimpanzees' social organization is often taken as a model for our ancestral lineage when it diverged from the other primates (Chapais 2008) - as a way to accommodate social complexity arising from highly individualized behavior (Read 2012, Fig. 4.4). The social complexity introduced through increased individuality (what Smaldino calls "individual differentiation"), was eventually accommodated within the hominin ancestry of Homo sapiens by shifting from social systems based on face-to-face interaction that characterize the non-human primates (which also leads to within-group, aggregated behavior upon which multilevel selection can operate) to relational based systems of social organization (Smaldino's institutionalized organization of differentiated individuals) that are culturally framed (Read 2012). The framing through cultural idea systems is not included in Smaldino's argument and is critical to our understanding of human systems of social organization (cf. Leaf 2009).

There is marked change in the ontological level at which selection operates and fitness is measured concomitant with the sequence going from genetic traits expressed individually and in isolation to traits expressed culturally and collectively. The sequence begins with fitness measured by the number of reproducing progeny, then when behavioral interaction among progeny is 
part of the trait, as occurs with biologically based altruistic behaviors, inclusive fitness becomes the measure of selection. With multilevel selection acting on traits expressed collectively through group structure (what Smaldino refers to as "collective behavior" or "aggregate traits"), group-derived fitness averaged over group members is assigned. Next are emergent traits, such as the linear, stable (e.g., Isbell \& Young 1993; Range \& Noë 2002), matrilineally inherited female dominance hierarchies (Kapsalis 2004) that emerge in many of the species making up the Cercopithecines from a female "placing" her biological daughter immediately below her in the dominance ranking (e.g., le Roux et al. 2011; see Read 2012 and references therein). Emergent traits, for Smaldino, provide transition from the uniformity of group behavior assumed in multilevel selection to organized, role differentiated behavior through which group level traits are expressed. Here, fitness is measured directly through the grouplevel trait.

Missing from this sequence, though, is the critical "next step" leading to the structure and organization of human societies (Read 2012; Read et al. 2009). Although Smaldino correctly places importance on systems of organization that incorporate role differentiated individuals in human societies, he does not discuss the fact that these systems of organization need not be emergent, but are often cultural constructions, such as the culturally formed kinship systems that provide structure and organization in human societies, especially in the small-scale societies that were the evolutionary precursors of large-scale human societies. Cultural kinship systems both define the societal boundaries and provide the structure and organization that establish the basis for the role differentiation that Smaldino discusses (Leaf 2009; Leaf \& Read 2012). The kinship terminologies that express the different systems of cultural kinship relations are not emergent, as research on the structural logic of kinship systems has demonstrated (e.g., Leaf \& Read 2012; Read 1984; 2001; 2007; 2010; Read et al. 2013). Terminologies are not the epiphenomena of already patterned behavior - as was assumed in some of the early research on human kinship systems and has been assumed in accounts of human evolution (e.g., Chapais 2008) - but are constructed idea systems (Leaf \& Read 2012) that provide conceptual organization for the small-scale societies from which present-day human societies have evolved. Kinship terminology systems have a generative logic to them that can be expressed through a "grammar," and differences among kinship terminology systems are derived from systematic differences in the generative logic of kinship terminologies (Read 2013).

Models of cultural evolution need to address not only the organizational aspects discussed by Smaldino, but also the complexity and structure of cultural idea systems that frame the systems of organization that are central to human societies. These cultural idea systems determine a framework within which behaviors take place and provide mutually understood meanings for behavior from the perspective of both agent and recipient that are critical for the coherence of human systems of social organization.

\section{Group-level traits are not units of selection}

\section{doi:10.1017/S0140525X13002999}

\author{
Carlos Santana ${ }^{\mathrm{a}, \mathrm{b}}$ and Michael Weisberg ${ }^{\mathrm{a}, \mathrm{b}}$ \\ alnstitute for Research in Cognitive Science, University of Pennsylvania, \\ Philadelphia, PA 19104-6228; ${ }^{\mathrm{b}}$ Department of Philosophy, University of \\ Pennsylvania, Philadelphia, PA 19104-6304. \\ csantana@sas.upenn.edu weisberg@phil.upenn.edu \\ http://www.phil.upenn.edu/ weisberg
}

Abstract: We argue that Smaldino has not established that group-level traits constitute a unit of selection distinct from selection on individuals, as group-level traits are neither replicators nor interactors. Moreover, we argue that Wimsatt's analysis of emergence and aggregativity supports an understanding of group-level and other emergent traits as explanatorily reducible to the individual level.

Smaldino argues that the division of labor may have influenced the evolution of social activity. We agree. However, he has not established that group-level traits constitute a distinct unit of selection. Moreover, Smaldino's argument that group-level traits are emergent, and therefore irreducible to the individual level, is unsound.

With respect to the first argument, we should follow Hull (1981) and distinguish between two senses of "unit of natural selection." Interactors are biological individuals such as organisms, which stand in ecological relations to the world. Replicators are biological units such as genes, which are copied from one generation to the next and preserved through evolutionary time. In claiming that group-level traits are units of selection, Smaldino may be claiming that they are replicators or that they are interactors. We will argue that they are neither.

It is unclear how group-level traits could act as interactors because they do not mediate interactions with the world for replicators that construct them. Smaldino himself seems to recognize this and never claims that group-level traits stand in ecological relations to the world. He does, however, emphasize the persistence and transmission of group-level traits, suggesting that he sees them as replicators. But persistence and transmission alone are not sufficient qualifications to be a Darwinian replicator. Darwinian replicators' fecundity must depend on their own nature, coming from something like protein structures or regulatory processes that they encode (Dawkins 1982). Because downstream products of replicators are not directly replicated themselves, phenotypic traits generally do not qualify as replicators despite their persistence and transmission. For example, human skin color is transmitted and reproduced over time, but is not regarded as a replicator. This is because we can fully account for the evolution of skin color by appeal to selection on populations of humans (interactors) and their genes or developmental systems (replicators). Group-level traits, analogously, are phenotypic traits whose transmission and reproduction are standardly accounted for in terms of individual-level replicators and interactors.

Smaldino disagrees, of course, arguing that group-level traits cannot be accounted for at a more basic level. To block the reduction of group-level traits to the individual level, he appeals to Wimsatt's (1997) analysis of emergence and aggregativity. An aggregative system is one in which the whole is a mere aggregate of its parts, whereas non-aggregative systems display emergent properties that cannot be accounted for by a simple summation of the properties of individual parts. For a system to count as aggregative, Wimsatt argues that the behavior of the system must be invariant under four different types of alterations to its parts: (1) rearrangement, (2) addition or deletion, (3) decomposition and recombination, and (4) linear amplification. Smaldino correctly notes that group-level traits are not likely to be invariant under any of these alterations, so they qualify as emergent in Wimsatt's sense.

We agree with Smaldino up to this point. However, Smaldino goes on to argue that because group-level traits are emergent, their evolution cannot be explained by selection at the individual level. This argument relies on a premise that we reject: that emergence implies irreducibility. This may be true for some emergent properties, but will not generally be true for the properties Wimsatt's test counts as emergent. In fact, a primary aim of the paper in which Wimsatt introduces the aggregativity test is to argue that emergence is fully compatible with reductive explanation. The fact that group-level traits pass Wimsatt's test for emergence does not therefore support the claim that group-level traits cannot be explained reductively in terms of selection on individual organisms or the units of culture of which they are composed.

Wimsatt's aggregativity test is also unsuitable for Smaldino's purposes because it identifies almost all systems as emergent. Even most physical properties (e.g., the combined volume of a mixture of different substances) are emergent in Wimsatt's 
sense, and nearly all social and biological systems will fail some, if not all, of the four conditions of aggregativity (Wimsatt 2000). This is problematic because it means that Wimsatt's test cannot distinguish group-level traits from the social systems Smaldino wants to contrast them with. For example, according to Smaldino, cooperation involves simply contributing resources to another individual but collaboration requires organized coordination and is thus an emergent property of a social system. However, many types of cooperation will vary along with the addition, deletion, and recombination of individuals, because these operations alter the relative distribution of resources. Thus, according to Wimsatt, cooperation is an emergent property. In this case, and many others important to Smaldino, Wimsatt's test cannot distinguish a special class of group-level traits.

Although Wimsatt's analysis of emergence does not support the claim that group-level traits are irreducible, it does illuminate another important aspect of explaining the evolution of social structures. By showing how emergence and reductive explanation can be compatible, Wimsatt helps us understand that we are not limited to methodologies that are either reductive and hostile to emergent properties, on the one hand, or non-reductive and friendly to emergence, on the other. Instead, understanding emergence along Wimsatt's lines opens up a middle path, a methodology that is reductive and nonholistic, but that uses this perspective to understand and explain emergent properties. Wimsatt calls this a reductive heuristic (Wimsatt 1997).

As we see it, the state of the art for cultural and social evolution modeling already follows this middle path. For example, deploying individual-based models (IBMs) is a fully reductive methodology in the sense that group properties are built out of individual interactions. Sophisticated IBMs include individuallevel relational properties such as relative spatial location and potential roles in collaborative effort. These are the very factors Smaldino emphasizes, and we suspect he approves of the use of IBMs in researching cultural evolution (e.g., Smaldino et al. 2012; 2013b). However, in individual-based modeling we can see how interactions lead to emergent properties without our having to abandon methodological individualism. For this reason, we remain unconvinced of the need to postulate irreducible group-level units of selection to generate scientific explanations of social interaction and evolution.

\section{Strong group-level traits and selection- transmission thickets}

doi:10.1017/S0140525X13003002

\author{
Jeffrey C. Schank \\ Department of Psychology, University of California, Davis, Davis, CA 95616. \\ jcschank@ucdavis.edu \\ http://www.agent-based-models.com
}

Abstract: I suggest that we should distinguish between weak and strong group-level traits. It is the latter that are especially problematic for developing models of cultural evolution. Using Campbell's notion of vicarious selection mechanisms, I argue that these mechanisms become intertwined with other group-level traits resulting in selectiontransmission thickets that are obstacles to modeling cultural evolution.

The theme of Smaldino's target article is the importance of developing a theoretical understanding of how group-level traits evolve. Group-level traits are not social organizations, but rather are phenotypic effects that emerge from social organizations. He focuses on a class of social organizations from which group-level traits emerge but which are not reducible to aggregates of individuals. Smaldino does this by analyzing social organizations using Wimsatt's (1997) four properties of aggregate systems. If a system is not reducible to an aggregate system, then it is an organization capable of producing emergent properties and behaviors. Smaldino argues that group-level traits emerge from social organizations that are strictly non-aggregative.

I believe that strict non-aggregativity is too strong a condition for social organizations capable of producing group-level traits. Strict aggregativity excludes paradigmatic examples of social systems, such as flocks of birds, that have emergent group-level traits. Although it is largely correct that flocks of birds have Wimsatt's (1997) aggregative properties (1) through (3), the failure to have property (4) - "there are no cooperative or inhibitory interactions among the parts of the system that affect this property" is crucial. I do believe, however, that properties (1) to (3) do demarcate an important class of social organizations and that it is fruitful to distinguish between weak and strong group-level traits that can emerge from these two classes of social organizations. The strong group-level traits emerge from strictly nonaggregative social organizations whereas weak group-level traits emerge from systems that may fail to have properties (1) to (3) but still have (4). Smaldino has done us a favor by pointing out that strong group-level traits should not be lumped in with weak group-level traits and require new models.

Indeed, strong group-level traits are especially problematic for the development of models of cultural evolution. Smaldino is well aware that strong group-level traits pose problems for developing models of selection and transmission of the social organizations that produce these traits. To reinforce and extend this point, I will devote the rest of this commentary to elaborating how difficult this problem is by connecting Smaldino's discussion of selection and transmission of group-level traits to Donald Campbell's (1956a; 1956b; 1960; 1974b; 1974c; 1975) notion of vicarious selection mechanisms.

Campbell (1975) recognized that in early human cultural evolution (e.g., the early cultural evolution of tools and weapons), the physical environment played an important role, and what tools and weapons evolved were directly affected by biological fitness. However, for the evolution of group-level traits (e.g., social organization, inhibitory moral norms, and beliefs in transcendent gods), Campbell's view was that biological evolution plays a minimal role at best. For many cultural traits, and especially group-level traits, vicarious selection mechanisms are required. For example, flintknapping practices for making stone tools and weapons can be selected and transmitted by imitating or learning from those who are successful flintknappers. Individual learning is a biologically evolved mechanism, which also serves as a vicarious selection mechanism for rapidly evolving cultural practices such as flintknapping.

Vicarious selection mechanisms require variation to operate on, but Campbell's (1960) requirement for blind variation-generating mechanisms in culture is too strong and not essential for models of cultural evolution (Sternberg 1998). Unlike genetic heritability mechanisms in biological evolution, there are many heritability mechanisms in cultural evolution. Campbell, like Smaldino, identified many retention and transmission mechanisms in culture such as imitation, individual learning, indoctrination systems, writing, and printing. There are, of course, others especially with the introduction of the Internet, and most importantly, they are still culturally evolving.

Mechanisms for selecting, generating variation, and transmitting cultural traits are often intertwined. For example, imitating the practices of one group also involves the selection of which practices are partially imitated. In addition, the selection and imitation of practices can introduce new variation resulting from imperfect imitation as with the cultural evolution of cargo cults. Unlike natural selection in which mechanisms of heritability, mutation, and selection are independent or nearly so, mechanisms of selection, variation, and transmission in cultural evolution can be properties of a single organization.

To this entanglement of mechanisms of selection, variation, and transmission, it must be added that as cultures evolve and become increasingly complex, new vicarious selection, variation, and 
transmission mechanisms (VSVTMs) evolve and appear at new levels in culture. The cultural evolution of military strategies, tactics, and organization by war games is one example. War games allow new tactics and strategies for warfare-group-level traits - to evolve without conducting actual war. War games are social organizations, but they are also VSVTMs for evolving new social organizations with tactics and strategies for actual wars. The evolution of VSVTMs does not end there; in modern militaries, computer-simulated war games introduce a new layer of VSVTMs (see Wagenhals \& Alexander 2001, for a discussion of simulated war games).

As cultures become increasingly complex, so do the number and kinds of VSVTMs, which evolve to facilitate rapid evolution. As they evolve, they can become deeply entwined with other social organization and VSVTMs. Thus, I would speculate that as cultures evolve with social organizations that have strong group-level traits, they also evolve VSVTMs with entanglements that produce selection-transmission thickets. For example, computer-simulated war games may be a group-level trait of war-game organizations that incorporate new individuals and technologies that facilitate the development of war-game simulations. These simulated games are group-level traits that function as VSVTMs for strategies and tactics used in war games and wars.

Selection-transmission thickets pose obstacles to modeling the cultural evolution of social organizations with strong group-level traits. They are often group-level traits that require cultural evolutionary explanation. Their component mechanisms (selection, variation, and transmission) are not aggregative (e.g., for the evolution of cargo cults, there is no clear distinction between selection, variation, and transmission). In population and quantitative genetic models of biological evolution, selection, variation, and transmission can be represented with additive or multiplicative models. For cultural evolution, the problem is how to cut through these thickets to develop tractable selection-transmission models at cultural levels.

\section{Group-level traits can be studied with standard evolutionary theory}

\section{doi:10.1017/S0140525X13003014}

\section{Thomas C. Scott-Phillips ${ }^{a}$ and Thomas E. Dickins ${ }^{b}$ \\ ${ }^{a}$ Evolutionary Anthropology Research Group, Durham University, Dawson

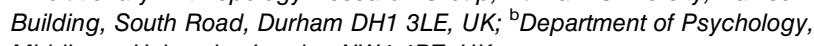 Middlesex University, London NW4 4BT, UK. \\ t.c.scott-phillips@durham.ac.uk t.dickins@mdx.ac.uk \\ http://thomscottphillips.wordpress.com \\ https://dissentwithmodification.com}

Abstract: Smaldino's target article draws on and seeks to add to a literature that has partially rejected orthodox, gene-centric evolutionary theory. However, orthodox theory has much to say about group-level traits. The target article does not reference or refute these views, and provides no explicit arguments for this narrow approach. In this commentary we: (i) give two examples of topics that the target article might and probably should have discussed (cultural epidemiology and the psychology of individual differences); and (ii) argue that the orthodox approach has much more to say about the emergence of group-level traits than the target article recognises, or gives credit for.

In recent years something of a divide has arisen in theoretical evolutionary biology. On the one side are those who argue that standard, gene-centric evolutionary theory is, in one way or another, incomplete or otherwise unsatisfactory (Laland et al. 2009; Pigliucci \& Müller 2010; Wilson \& Wilson 2007). On the other side are the silent majority of evolutionary biologists, who continue to work, productively, with standard evolutionary theory, and do not see any particular reason for change. As critics of orthodox theory acknowledge, "The commonest reaction to our explanations is something along the lines of 'But that is already understood as part of the Modern Synthesis anyway" (Pigliucci \& Müller 2010, p. 4).

The revolutionary position has, however, received slightly wider acceptance within one particular community: evolutionary perspectives on humans and human behaviour. This is not to say that standard, gene-centric evolutionary theory has been rejected by that community. On the contrary, many researchers continue to use it, and the literature includes vigorous defenses of it, and criticism of the purported alternatives (e.g. Dickins \& Rahman 2012; Pinker 2012; Scott-Phillips et al. 2011; West et al. 2011). Nevertheless, there is a sizeable group of researchers in this area that have been persuaded that orthodox evolutionary biology does not on its own provide a sound theoretical basis for the evolutionary study of human behaviour, culture, and society (e.g. Laland et al. 2007; Richerson \& Boyd 2005; Wilson \& Sober 1994). As a result, "the sharpest divide between evolutionary students of human social behavior is between ... Strict NeoDarwinians and Expanded Synthesis Evolutionists" (Richerson 2012).

There is now a sizeable literature built around this partial rejection of evolutionary orthodoxy. In particular, there are considerable bodies of work that argue: (i) that the evolution of many human social behaviours is best explained in terms of cultural multilevel selection (despite the fact that there is no formal justification to consider the cultural group as a unit of selection [Gardner \& Grafen 2009; West et al. 2011]); and (ii) that cultural evolution and other forms of extra-genetic inheritance change the Darwinian process to such an extent that they render orthodox evolutionary theory ill-suited for the study of humans, human behavior, and human culture, and hence that its utility in this domain is limited, perhaps only to special, idealized cases.

Smaldino's target article implicitly accepts these arguments as a premise, poses a new question for them (How can we explain group-level traits?), and, within this framework, considers some possible approaches and answers to that question. It thus almost ignores more orthodox evolutionary theory, in which culture is a proximate mechanism (but not "just" or "merely" a proximate mechanism), and approaches to cultural evolution and grouplevel phenomena that have been developed within this orthodox perspective. Some of this work is extremely relevant to Smaldino's questions, and indeed even provides some answers, yet it is not at all cited. By way of illustration, we here highlight two examples. There are others, but we have only limited space.

The first is the work of cultural epidemiologists, who have developed a rich literature of both theory and data to explain the existence and emergence of group-level traits. In this framework, the spread of cultural traits through a population is explained by the fit of the trait to the natural dispositions of the human mind (or the local cultural ecology), just as parasites spread to the extent that they fit the natural physiologies of host organisms (Sperber 1996). By virtue of this process, cultural traits gravitate towards certain forms (called "attractors") and away from others (Sperber \& Hirschfeld 2004). As such, this research agenda explains culture in terms of its fit with the natural, evolved dispositions of the human mind, and in doing links cultural evolution with standard evolutionary approaches to human psychology. This approach has been successfully used to study the emergence of numerous cultural traits, such as religious beliefs, kinship systems, legal norms, and others, which are variously either the sort of group-level traits that Smaldino is interested in, or the institutions that give rise to them (e.g., Atran \& Norenzayan 2004; Bloch \& Sperber 2002; Boyer \& Petersen 2012). Either way, cultural epidemiology has a great deal to offer Smaldino, but his target article does not make use of it.

The second example of research that can potentially address some of the issues raised in the target article, but from a more orthodox perspective, is the psychology of individual differences (Nettle 2007). One hypothesis, which follows from and is productively studied within standard evolutionary theory, is that 
individual differences (in, e.g., personality, intelligence, neuroticism, and so on) are adapted, niche-specific life-history strategies, and can be the product of an individual plasticity that has been selected in order to maximise inclusive fitness across varied environments (Buss \& Greiling 1999; Nettle 2006). The growing importance of group living in human evolution will have brought with it trade-offs around competition for resources, and with it sufficient pressure for selection of niche-specific life history strategies; in other words, individual differences. Division of labour within the group, becoming a specialist within the group structure, could readily act to maximize individual fitness, but the cost would be the loss of possible independence should the group disintegrate. A snapshot observation of this structure might subsequently lead an observer to conclude the group has emergent properties. However, the ultimate causal explanation would still be the individual maximization of inclusive fitness, as elaborated by standard evolutionary theory.

In sum, because Smaldino has chosen to conduct his discussion almost entirely within the context of a literature that has rejected at least part of orthodox theory, the target article fails to recognise that there are other literatures that potentially offer simple, elegant explanations of the phenomenon it is concerned with. In fact, we think it quite possible that the integration of the two areas we explicitly mention above could provide an extremely rich, synthetic explanation of group-level traits, but this possibility is not entertained. On the contrary, the very existence of alternative, orthodox ways of thinking about the topic at hand is not even mentioned.

Smaldino asserts that "an evolutionary theory of culture is here to stay" (sect. 1, para. 1). We agree, and welcome this. However, there is no good reason why that theory can or should ignore other existing, productive, and more evolutionarily orthodox literatures, especially those that have much to say about the concerns of cultural evolution theorists. (This is true even if we put to one side the question of whether the partial rejection of evolutionary orthodoxy that exists in much of the cultural evolution literature is justified.) Indeed, these other literatures have made and tested a number of explicit predictions about group-level traits and/or closely associated phenomena. In contrast, exactly what predictions follow from Smaldino's analysis is not clear.

\section{Language as an emergent group-level trait}

doi:10.1017/S0140525X13003026

Lan Shuai $^{\mathrm{a}}$ and Tao Gong
a
${ }^{\mathrm{a}}$ Department of Electrical and Computer Engineering, Johns Hopkins
University, Baltimore, MD 21218; ${ }^{\mathrm{b}}$ Department of Linguistics, University of
Hong Kong, Pokfulam Road, Hong Kong.
susan.shuai @ gmail.com
http://www.ece-jhu.org/
gtojty@gmail.com
http://www.linguistics.hku.hk/

Abstract: Following Smaldino's definition, we claim that language is also an emergent group-level trait, and propose two facets (human groups tend to organize in a way to efficiently trigger language and linguistic interactions can render formation of certain social organization) to verify this statement, both of which also provide a general framework to address the future work about group-level traits.

In the target article, Smaldino proposed the defining characteristics of an emergent group-level trait. Apart from the examples in the target article, we point out that human language, as a prominent sociocultural phenomenon in human communities, is also an emergent group-level trait, considering the facts that (a) human groups tend to organize in such a way to efficiently trigger a communal language and (b) linguistic interactions can influence the formation of social organization having certain properties. In line with Smaldino, these facets demonstrate that language is more than a collective behavior dependent on specific organizations of differentiated individuals. In this commentary, based on empirical evidence and computer simulation, we illustrate how to verify a sociocultural phenomenon like language as an emergent group-level trait from these two aspects.

On the one hand, according to the empirical evidence (Newman 2003), social communities formed via language-related interactions tend to show a scale-free characteristic (Barabási 1999); these communities usually exhibit power-law degree (the number of social connections an individual has with others) distributions, and the $\lambda$ values of those distributions are often around 2.0 (e.g., the telephone call network, $\lambda=2.1$ or the e-mail message network, $\lambda=2.0$ ). Whether such social organization is correlated with language evolution can be explored by computer simulation. For example, we can adopt the lexicon-syntax coevolution model (Gong 2009; 2011) tracing the origin of both lexical items and basic word orders in a population and define a power-law distributed social popularity (the probability for an individual to participate in communications has a power-law relation with its rank in the group) to mimic such social organization. Then, by adjusting the population size and the $\lambda$ value in the social popularity, our simulations show that: when $\lambda=1.0$, a communal language with a high mutual understandability $(M U$, the proportion of semantic expressions that all individuals can produce and accurately interpret using their linguistic knowledge) can be efficiently triggered across various population sizes, but if $\lambda$ has bigger values, $M U$ starts to drop significantly, particularly in bigger groups (see Fig. 1). Mathematically speaking, $\lambda=1.0$ in this social popularity corresponds to $\lambda=2.0$ in the power-law degree distributions in those social communities. These results clearly reveal the correlation between language and human communities: a particular social organization can efficiently trigger a communal language with a high $M U$.

On the other hand, just like other cooperative behaviors, linguistic interactions and mutual understandability can form social bonds among individuals. Such local bonds may trigger some social organization at the group level. To illustrate this, we conduct another study also based on the lexicon-syntax coevolution model. Here, we assume that: (a) each individual has a predefined local-view and only interacts with others falling into his or her local-view; (b) individuals adjust the weights (initially 0.0) of their links to other individuals, based on the success or failure of previous communications with others; and (c) once the weight of a link exceeds a threshold (say, 0.5), a permanent link is formed, and this individual will prefer interacting with those he or she permanently connects to. These assumptions resemble the friendship formation based on common interests in human communities.

Under such assumptions, our simulations show that a social structure possessing the small-world characteristic (Watts 1999) can be gradually formed, along with the origin of a communal language with a high $M U$. We also observe a correlation between $M U$ and the local-view size (see Fig. 2): with the increase in local-view size in different sets of simulations, some individuals become more centralized than others and participate in more communications to spread their linguistic knowledge, thus increasing $M U$ of the group; however, too much centralization around few individuals fails to further increase $M U$, because apart from these individuals, others may not have sufficient opportunities to interact with each other, and as a consequence, this may affect the spread of linguistic knowledge and $M U$ of the group. These results not only echo the findings in Figure 1, but also show that certain social organization or properties can be partially ascribed to the group-level trait such as language. According to Smaldino, this is a key feature to distinguish a group-level trait from a simple collective behavior.

In the above two aspects, apart from the lexicon-syntax coevolution model, studies based on other language models (e.g., Baronchelli et al. 2006; Puglisi et al. 2008) can also examine the 
(a)
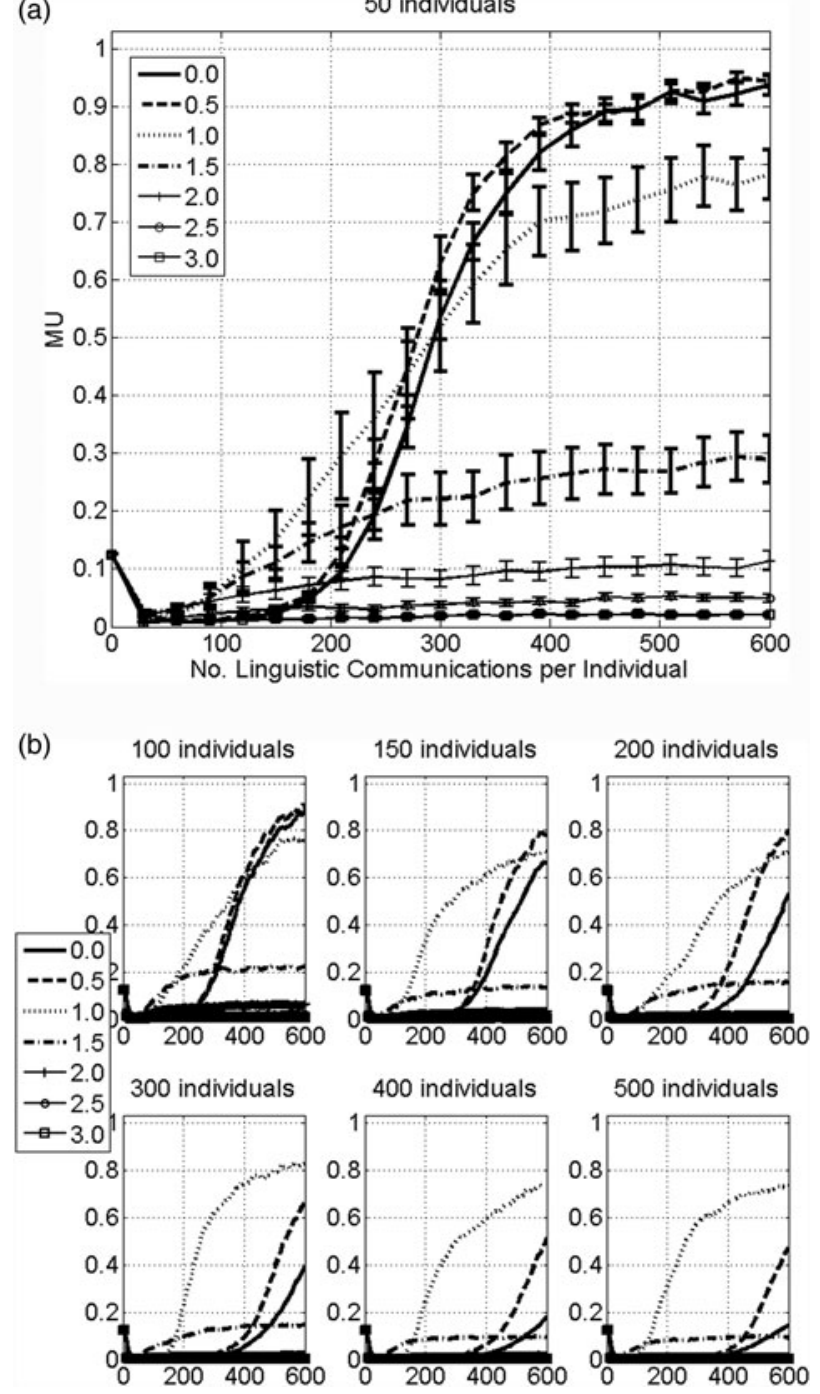

Figure 1 (Shuai \& Gong). Mutual understandability $(M U)$ under Various Social Popularities in a 50-individual Population (a) and Populations Having Other Sizes (b). Each line denotes the average $M U$ (over 20 simulations) under a social popularity with a particular $\lambda$. Error bars denote standard errors (because of size, error bars in (b) are omitted). It is shown that when $\lambda=$ 1.0, the dynamics of language origin (indicated by $M U$ ) is not only similar across different population sizes, but also optimal compared to those under other $\lambda$ values.

effects of structural features on language evolution (e.g., Baronchelli et al. 2009; Dall'Asta et al. 2006; Gong et al. 2012a; 2012b). Apart from social interactions, it is shown that combinations of different forms of cultural transmission can also affect language evolution across generations of individuals (e.g., Gong 2010). Moreover, models of other social activities (e.g., cooperation, collaboration, labor division, leadership formation, etc.) can also be adopted in those simulation studies. Exploring the dynamic correlation between sociocultural organization and language (or other group-level traits), these studies offer a general computational framework to evaluate whether a sociocultural phenomenon is qualified as an emergent group-level trait. This framework focuses on individual behaviors and group structures, as well as on the correlation between emergent group-level traits and sociocultural organization patterns. According to Smaldino, this framework is very promising for the future work concerning group-level traits in ecological and evolutionary contexts.

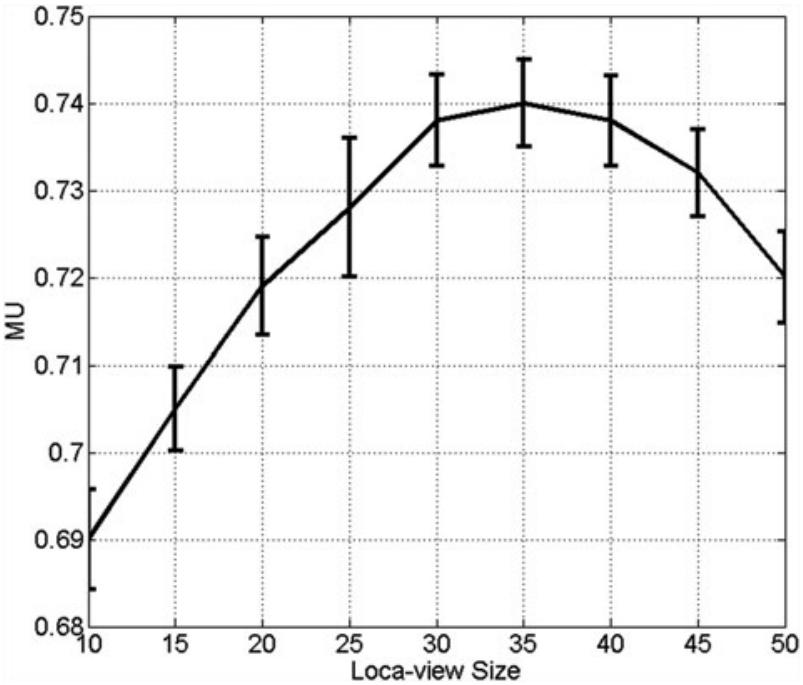

Figure 2 (Shuai \& Gong). Mutual understandability $(M U)$ vs. Local-view Size (when local-view size $=50$, each individual can view all members in the group). Each simulation has 50 individuals and 500 communications, and the adjustment on link weight is 0.01 . The results are averaged over 20 simulations. Error bars denote standard errors.

ACKNOWLEDGMENT

This work is supported by the Seed Fund for Basic Research of the University of Hong Kong.

\section{Why religion is better conceived as a complex system than a norm-enforcing institution}

\author{
doi:10.1017/S0140525X13003038
}

\section{Richard Sosis and Jordan Kiper}

Department of Anthropology, University of Connecticut, Storrs, CT 06269-1176. richard.sosis@uconn.edu jordan.kiper@uconn.edu

http://www.anth.uconn.edu/faculty/sosis/

Abstract: Although religions, as Smaldino demonstrates, provide informative examples of culturally evolved group-level traits, they are more accurately analyzed as complex adaptive systems than as normenforcing institutions. An adaptive systems approach to religion not only avoids various shortcomings of institutional approaches, but also offers additional explanatory advantages regarding the cultural evolution of group-level traits that emerge from religion.

The target article is an important contribution to the study of cultural evolution and an impressive account of the emergence of group-level traits. In Smaldino's view, norm-enforcing institutions and cultural narratives are necessary for maintaining suites of cultural traits, such as organizational patterns, behavioral norms, and cooperative decision-making heuristics. If these traits are maintained over time, they will potentially contribute to the emergence of group-level traits. These are the phenotypic effects of social organization and interdependent collaborations that are made possible only when individuals with differentiated roles are structurally cooperative and socially coordinated. Furthermore, because religion is one of the most effective mechanisms for transmitting whole suites of cultural traits, such as hierarchies that reinforce the group's social organization, it is one of the most important constituents of traits at the group level.

We are in general agreement with Smaldino's account, but we think he has overlooked two crucial points about religion: its unique role in stabilizing prosocial norms and its distinct 
effectiveness at transmitting suites of cultural traits. Approaching religion as an institution, as Smaldino does, can lead to worthwhile insights about the social structures that support religion and the function of religion as a cultural organization. However, analyzing religions as complex systems more accurately reveals the structures that maintain religions, as well as the traits that emerge from them. Religious systems consist of core interdependent features, such as rituals, supernatural agent beliefs, and myths, which evolved independently but at some point in human history began to co-occur and coevolve regularly and cross-culturally (Alcorta \& Sosis 2005; Purzycki \& Sosis 2009; Sosis 2009). As anthropologists observe, elements of religious systems interact in complex ways, resulting in emergent properties (e.g., Geertz 1973) and creating communities with shared customs, cognitive schemas, historical memories, and identities.

Although we are critical of Smaldino's characterization of religion as a norm-enforcing institution, we believe that an adaptive systems approach is consistent with his argument for the emergence of group-level traits and provides various explanatory advances to his conception of religion. We address each of these advantages in what follows. In doing so, we hope to show that, from a cultural evolutionary perspective, religion is better conceived as a complex adaptive system rather than a norm-enforcing institution.

First, understanding religion as a complex adaptive system clarifies how religions succeed at transmitting suites of cultural behaviors while concurrently adapting to varying socioenvironmental conditions. Religious systems achieve this apparent opposition by maintaining hierarchies of religious discourse, in which adherents focus on core statements of belief that remain unchanged, even as interpretations of those statements change over time. Accordingly, adherents typically accept novel interpretations of fundamental postulates as being reifications of eternal or personally relevant truths, while taking little notice of the modifications to religious rules and social norms with each generation (Rappaport 1999; Sosis 2011).

Second, the complex adaptive systems approach exposes the mechanisms by which religious systems achieve extensive cooperation and coordination, as observed experimentally and ethnographically (e.g., McKay et al. 2013; Sosis \& Ruffle 2003; Xygalatas 2013). One such mechanism is religious signaling systems: religious activities are costly and thus serve as honest signals that enable and sustain trust, allowing groups to cooperate and coordinate socially (Irons 2001). In other words, by performing religious activities, adherents reinforce cooperative norms and signal their commitments to the group, which in turn provides the necessary levels of trust to overcome collective action problems and to maintain group-level traits. However, signaling theory advances Smaldino's argument on other fronts as well. Because signaling theory recognizes that systems can remain stable despite some deceptive signalers (Johnstone 1997), it anticipates the inherent variation within and across religious communities that Smaldino presumes (Wildman \& Sosis 2011). It also explains why adherents are highly attuned to signaling variations: they carry respective fitness consequences. Finally, given that signals require arenas of display and reception, signaling theory emphasizes the importance of social and cognitive niches created by religious communities (Bulbulia \& Sosis 2011).

Third, the complex systems approach can explain the variation in social structures across religious systems, including religious variations among bands, tribes, chiefdoms, and states. Although religious systems consist of a core set of recurring features, cultural variation arises from how these elements interact within local ecologies to produce specific practices, beliefs, and structures. For example, although many Christian groups maintain an office of ministry, as Smaldino describes, it is hardly a universal or even a common feature of most religious systems. Rather, ministries emerge under specific conditions, such as when groups can benefit from economies of scale (Sosis 2003, p. 115), which are more common in state systems.

In addition to these three advances, the complex systems approach also overcomes several inherent limitations to
Smaldino's understanding of religion as a norm-enforcing institution. First, Smaldino situates social norms outside of religious systems, such that religious institutions act on norms that emerge independently, which is unreflective of human history. The systems approach, in contrast, recognizes that religion was not a separate and well-defined arena of social activity for most of human existence. Rather, religious systems permeated all aspects of social life, and thus norms coevolved with the system itself. Second, religious systems not only enforce norms, but they also naturalize them (Rappaport 1999). Because religion is comprised of cognitive, behavioral, and developmental elements and regularly activates human senses - it fully engages practitioners, making social norms feel correct and natural. Lastly, although we share Smaldino's concerns about reductionism, it is necessary to recognize that reductionism is nevertheless essential for uncovering the constituents of religion. However, the complex systems approach avoids the pitfalls of reductionism by emphasizing that selection operates on the system itself, not its constituent parts (Sosis 2009).

To conclude, we stress that we are not offering a competing alternative to Smaldino's account, but rather a helpful extension of his approach. Indeed, we are curious to hear from Smaldino whether the complex adaptive systems approach can complement the valuable extensions to cultural evolutionary theory that he has offered in the target article.

\section{Replicators, lineages, and interactors}

\author{
doi:10.1017/S0140525X1300304X
}

\section{Daniel J. Taylor and Joanna J. Bryson \\ Department of Computer Science, University of Bath, Bath BA2 7AY, UK. djt20@bath.ac.uk jjb@cs.bath.ac.uk \\ http://www.cs.bath.ac.uk/ jjb}

Abstract: The target article argues that whole groups can act as interactors in an evolutionary process. We believe that Smaldino's discussion would be advanced by a more thorough analysis of the appropriate replicators and lineages for this model. We show that cultural evolution is necessarily a separate process from cultural group selection, and we also illustrate that the two processes may influence each other as demonstrated by an agent-based model of communicating food-processing skills.

Smaldino argues that human groups can act as cohesive units to the extent that they can be considered interactors in an evolutionary model. To briefly review standard evolutionary theory, an evolutionary process requires three things-replicators, interactors, and lineages. Replicators are entities that pass on their structure intact into successive generations. Interactors are entities that interact as a whole with their environment, leading to differential replication (selection). Lineages are entities that persist indefinitely whether in the same state or in one altered by the process of replication.

We believe that Smaldino's discussion can be advanced by a more thorough analysis of the appropriate replicators and lineages for this model. In human cultural evolution, there are at least two sets of things that might form lineages. The first is a set of humans who interact at a specific location, for example, a company or a village. The second is the components of the culture itself - for example, the set of ideas and practices that make up biology or physics. Obviously these two sorts of lineages will not always be perfectly aligned.

Cultural group selection by standard definition is about the first sort of lineage (sets of people at a location). However, the notion of memetics suggests that there may also be a second replicator system - the lineage formed by the memes themselves. These might sensibly be expected to produce "emergent, group-level traits" and would be mostly independent of cultural group selection, although sets of people might well exploit its consequences as a defining feature of their group identity. 
If we adopt the first option, we see that cultural group selection concerns the fraction of groups within a specific area that are of a specific type. However, this head-counting method of reckoning group selection does not map cleanly to what we would like to call adaptation - the thing that natural selection seeks to explain. For example, we would not want to say that Inuit culture is "less adapted" than Roman culture because there were at one point more Romans.

If we adopt the other option and refer to culture itself as the lineage, then the culture itself can evolve because the replicators are the ideas and practices that exist within that culture. However, if the culture is the lineage, we cannot say that it evolves when it takes more territory, in the same way that a species does not evolve with more individuals. Adaptation is presently understood to be about changes in the frequency of replicators, not about absolute numbers of interactors. In sum, cultural evolution (changes of practices within a group) is necessarily a separate process from cultural group selection (changes of the frequency of group-types at a specific location).

We can illustrate these points with a spatial agent-based model of cultural accumulation of knowledge about food-processing skills (Čače \& Bryson 2007). In this model there are two variants of a species: free riders who exploit knowledge but never share it and altruists who communicate knowledge with any other nearby agent (perhaps just by failing to conceal their food-processing skills). Knowledge enters the system at a fixed rate of chance discovery, such that each agent has a small chance of discovering a new food source in its own life. Because of constraints placed on lifespan and on the rate of communication, "communities" of neighbouring agents form with expertise in a small fraction of the available skills the environment affords, though this fraction is still larger than the maximum of one skill any agent might learn on its own. When two communities encounter each other by chance, there is a brief surge in population, as both groups quickly learn about the super-set of their food-processing skills. But this process is not evolution; it is only a temporary advantage from happenstance exchange. Future generations cannot sustain the level of cultural accumulation because there is no meta-behavioural (e.g., deliberate teaching) acquisition of skills, nor any change in the biological factors (e.g., lifespan) that determined the likely number of items that can be transmitted from one generation to the next.

Nevertheless, as with most viscous spatial models of altruistic behaviour, there is adaptation in the biological sense. Altruists outcompete free riders, because they are more likely to know about food sources, because they are more likely to live near knowledge sharers - their relatives. This is a simple function of being born by your mother and taking time to move, a process understood by Hamilton (1964) but sometimes overlooked in simplistic modelling (Sober \& Wilson 1998).

The upshot for Smaldino's target article is that we have slightly corrected his use of language, but have largely supported his main claim, including providing evidence in the form of a formal agentbased model.

\section{The collaborative emergence of group cognition}

\section{doi:10.1017/S0140525X13003051}
Georg Theiner $^{\mathrm{a}}$ and John Sutton ${ }^{\mathrm{b}}$
${ }^{a}$ Department of Philosophy, Villanova University, Villanova, PA 19085;
${ }^{\mathrm{b}}$ Department of Cognitive Science, Macquarie University, Sydney, NSW 2109, Australia.
georg.theiner@villanova.edu
http://villanova.academia.edu/GeorgTheiner
john.sutton@mq.edu.au
http://www.johnsutton.net

Abstract: We extend Smaldino's approach to collaboration and social organization in cultural evolution to include cognition. By showing how recent work on emergent group-level cognition can be incorporated within Smaldino's framework, we extend that framework's scope to encompass collaborative memory, decision making, and intelligent action. We argue that beneficial effects arise only in certain forms of cognitive interdependence, in surprisingly fragile conditions.

Smaldino rightly distinguishes genuinely emergent group organization from mere aggregation, pointing to the active collaboration of individuals with different capacities as the key form of cooperation. We offer two friendly but important extensions. We incorporate group cognition into Smaldino's framework; and we see the conditions under which structured differentiation is beneficial as more fragile than he acknowledges. These modifications encourage integration of work on the cultural evolution of group-level traits with substantial research traditions on distributed cognition, organizational psychology, and collaborative recall.

On standard views in cognitive science, cognition is strictly an individual-level achievement. "Social" cognition is thought to occur when people think about social phenomena or when social stimuli trigger cognitive processes. But cognitive processes are conceived in nonsocial terms. If group cognition is countenanced, it is understood atomistically, as the aggregate output of individual cognition plus social processes of combination. Little attention is paid to collaborative interdependence as the hallmark of emergent group-level cognition, as expressed in the Gestalt maxim that "the whole is greater than the sum of its parts."

This attractive but puzzling concept is clarified in a large body of rigorous experimental research on group problem solving in social and organizational psychology (Laughlin 2011) that is surprisingly neglected both in Smaldino's presentation and in cognitive science at large. In one key work, Steiner (1966) distinguished five types of group tasks. In additive and compensatory tasks, group members do not interact in producing an outcome. In additive tasks, the group outcome is indeed the sum of the member contributions. In compensatory tasks, the group outcome is a statistical average of individual solutions. The proper aggregation of estimates or predictions can yield greater information gains than the sum of individual contributions (Bettencourt 2009), as in the "wisdom of crowds" (Surowiecki 2004). But such crowd intelligence is largely aggregative in the sense of Wimsatt (1986), as Smaldino argues.

Although conjunctive, disjunctive, and complementary tasks require interactions among group members, only the latter are collaborative in a richer sense. Conjunctive and disjunctive tasks are end points on a spectrum of how many group members must succeed individually for the group to succeed. A disjunctive task, for example, would be a group working on a sudoku, where the group succeeds if any of its members solves the puzzle. Here, the role of social interactions is a matter only of recognizing and adopting a solution found by any one member. In complementary group tasks, on the other hand, members coordinate and combine their diverse knowledge, abilities, and cognitive resources into a collective, organization-dependent outcome that no individual could have produced alone. Psychological processes studied from this group-level perspective include problem solving (Larson \& Christensen 1993), collective induction (Laughlin \& Hollingshead 1995), the development of transactive memory systems (Wegner 1986), and creativity (Hargadon \& Bechky 2006).

Likewise, the "distributed cognition" framework studies collaborative, dynamically evolving work practices mediated by the use of tools and representational instruments and carried out in environments that provide a rich organizational structure (Hutchins 1995b; Perry 2003; Sutton 2010). It borrows from traditional cognitive science an emphasis on processes of creating, transforming, and propagating representational states, but views them as part of larger cognitive ecologies that involve the coordination 
of resources across people, tools, and shared environments. Complex collaborative tasks that have been analyzed as distributed cognitive systems include maritime navigation crews (Hutchins 1995b), emergency rescue management (Garbis \& Waern 1999), theatrical practices in Elizabethan drama (Tribble 2005), bioengineering labs (Nersessian 2006), and crime scene investigation (Baber et al. 2006). Expanding Smaldino’s framework, we argue that these cases exemplify emergent group-level cognition (Sutton et al. 2010; Theiner 2013; Theiner \& O'Connor 2010).

Our concept of emergent group cognition differs from the "assembly bonus effect," when "the group is able to achieve something collectively which could not have been achieved by any member working alone or by a combination of individual efforts" (Collins \& Guetzkow 1964, p. 58; cf. Larson 2010). Firstly, an assembly bonus effect can occur without emergent group cognition. The "wisdom of crowds" critically depends on the lack of collaborative interdependence. It requires, ideally, that individual decision makers are connected only through suitable information aggregators such as market pricing, but otherwise do not influence each other's judgments.

Secondly, emergent group cognition does not necessarily produce assembly bonus effects. This is why Smaldino's claim that "structured differentiation is often beneficial to group success" (sect. 3, para. 7), while appropriately correcting standard views, needs some qualification. For example, though shared remembering in dyads or groups is a ubiquitous human activity, experimental studies of collaborative recall find that groups often remember less than the sum of their parts (Harris et al. 2013; Weldon \& Bellinger 1997). The most common explanation for such collaborative inhibition is that hearing other people recall disrupts individuals' idiosyncratic mnemonic strategies (Basden et al. 1997). The fact that collaborative facilitation is surprisingly hard to find experimentally (but see Harris et al. 2011; Meade et al. 2009) suggests not that emergent group cognition does not occur, but that the conditions under which it is beneficial are surprisingly fragile. As Smaldino notes, the history of group organization matters, as does the structure of differentiated expertise. A further key factor is the fine-grained nature of the communicative interactions in active collaboration among group members (Sterelny 2012; Sutton 2013). This point can be neglected in social combination approaches in small group research, which tend to be output- rather than process-oriented and concerned mostly with intellective tasks where comparison with traditional baseline models (e.g., truth-wins, better-than-bestmember) makes sense. Apart from lacking ecological validity, such narrow focus can lead us to misconstrue the functions of real-world group cognition. An important function of shared remembering, for example, is to reinforce social bonds, by merging disparate memories into a stable rendering of shared past experiences (Barnier et al. 2008; Hirst \& Manier 2008; Hirst \& Echterhoff 2012). Recognizing the beneficial effects of collaborative interdependence requires that we conceptualize "group success" more broadly.

This concept of emergent group cognition can centrally inform ecologically realistic studies of the coevolution of minds, groups, and cultures.

\section{Coordination, cooperation, and the ontogeny of group-level traits}

doi:10.1017/S0140525X13003063

Timothy Michael Waring and Sandra Hughes Goff

School of Economics and Sustainability Solutions Initiative, University of Maine, Orono, ME 04469.

timothy.waring@maine.eduｓandra.goff@maine.edu

www.umaine.edu
Abstract: Group-level traits (GLTs) clarify the necessity of cultural multilevel selection theory. We propose a revised definition of the GLT concept. We also highlight the need to better understand the interplay between the dimensions of cooperation and coordination in the ontogeny of GLTs.

We find Smaldino's argument lucid and compelling. His clear delineation of group-level traits (GLTs) pinpoints the sorts of social complexity that cannot be explained by selection of individuals or kin and highlights the importance of coordination, in addition to cooperation, as a fundamental determinant in cultural evolution. We think the examination of GLTs should be taken further and that by doing so the concept may help catalyze advances in fields that draw on evolutionary theory.

Smaldino's call for a "between-levels perspective" (sect. 7, para. 2) parallels one for a mesoeconomic approach (Dopfer 2011; 2012) from evolutionary economics. Mesoeconomics is often considered as a placeholder for economic studies that do not fit neatly in either micro or macro categories (Dopfer 2011). However, if economics can grow to include Smaldino's culturally transmissible GLTs subject to natural selection, we feel that mesoeconomics could "gain an independent and genuine place in the architecture of economics" (Dopfer 2011).

However, first, GLTs may need to be redefined. Smaldino argues that GLTs are the phenotypic effect of social organization, rather than that organization itself. We agree that group-level social organization does have "phenotypic" effects (e.g., the music of a rock band) and that those phenotypic effects are subject to natural selection. However, if we call the phenotypic effect of social organization the "trait," how then do we talk about different social structures with similar phenotypic effects, or the multiple phenotypic effects of a single type of social organization? In biology, the concept of pleiotropy refers to the condition in which one gene generates multiple phenotypic effects. Such a thing is surely also possible with social organization. This issue makes the "phenotypic effect" definition of GLT awkward to apply. For example, the music played by the rock band may be recorded and played back. We would all agree that the performance is the phenotypic effect of the rock group's organization, but if we use Smaldino's definition, then the recording of the performance would also be considered a GLT. But certainly, the recording is not a group-level organizational trait, but the output of one. Defining GLTs as the details of social organization itself solves a number of issues with the concept.

This redefinition has the benefit of simplifying how we conceptualize institutional selection. If a GLT is an organizational structure, then we can see that group-level phenotypic effects can be the result of individual-level traits, emergent GLTs, aggregate group behaviors, or any combination thereof. Discussions of group selection tend to focus on aggregate group-level effects exerting influence on the prevalence of cooperative individuallevel traits within a population. GLTs are behaviors that cannot be enacted by a single individual and are built on interdependencies between individuals (e.g., zone defense, a queue, square dance). For this reason, a GLT can only exist when organization matters. Organization is only likely to matter in the context of preexisting behavioral heterogeneity and/or culturally transmitted differentiated social roles. Smaldino's general insight is that models of cultural evolution stand to be advanced substantially by the addition of these types of heterogeneity. We also believe that such a consideration will necessarily help the study of the development, or ontogeny, of social structures.

In examining the social ontogeny of structures, coordination and cooperation leap out as central dimensions. Smaldino suggests that both are necessary components of GLTs, but he stops short of defining them as hard requirements. Both cooperation and coordination influence the development of a social structure and bear on Smaldino's aggregate versus emergent distinction.

Some social structures may begin their development with a set of differentiated actors and then come to develop cooperation over time. An archetypal example of such a structure is the 
market economy in which individuals possess differentiated resources and skills to trade. In this example, each person has many deficits and one surplus, so most random pairings can lead to mutually beneficial exchange with only the most limited amount of cooperative action. Thus, the social structure of the market is an important boundary sitting between aggregate group behaviors such as flocking, on one hand, and Smaldino's emergent, cooperative, role-differentiated structures, on the other.

Other social structures may start with a cooperative set of individuals and develop role differentiation over time. As an example, a cooperative group happens upon a novel challenging scenario, requiring their combined effort, such as hunting a new and dangerous type of prey. Here, high levels of preexisting cooperation facilitate the development of differentiated and interlocking roles, helping the group become successful. A less cooperative group may not have had the same success.

These two archetypal institutions are further differentiated by their ongoing ontogenetic paths. In the market example, coordination can be selfish and mutually beneficial. The mutual benefit and low requirements for cooperative investment tend to make such distributed social structures resilient. Coordination based on preexisting differences is easy to develop and takes no work to maintain. Moreover, high levels of cooperation do not necessarily follow. The market example contrasts with the cooperative group scenario in which high levels of cooperation potentiate high levels of integrated and coordinated action. It is no surprise that groups that are both more coordinated and more cooperative can accomplish more complex and demanding feats. NASA would not have been able to put humans on the moon with coordination alone; cooperation and central planning are required. But the ontogenetic order of cooperation and coordination in the development of a social structure, and the social preconditions that determine that order, may determine which type of structure emerges. This begs the question: When and how do market-like organizations become more highly cooperative and tightly integrated and vice versa?

Can a coordinated, weakly cooperative organization become a more coordinated, highly cooperative one? Do economic integrations easily give rise to political and social integration? In 2013, with the recent challenges faced by the European Union, this question is not idle speculation. The scale and scope of our political-economic challenges highlight the urgent need for this research to be extended to socioeconomic theory and policy.

\section{Groups as units of functional analysis, individuals as proximate mechanisms}

\section{doi:10.1017/S0140525X13003075}

\section{David Sloan Wilson}

Evolution Institute and Departments of Biology and Anthropology, Binghamton University, Binghamton, NY 13902-6000.

dwilson@binghamton.edu

http://evolution.binghamton.edu/dswilson/

Abstract: Whenever selection operates at a given level of a multitier hierarchy, units at that level should become the object of functional analysis, and units at lower levels should be studied as proximate mechanisms. This intuition already exists for the study of genes in individuals, when individuals are the unit of selection. It is only beginning to be applied for the study of individuals in groups, when groups are the unit of selection. Smaldino's target article is an important step in this direction with an emphasis on human cultural evolution, but the same algorithm applies to all multilevel evolutionary processes.

Two major themes have characterized multilevel selection theory from Darwin to the present (Wilson 1997). One theme is centered on the concept of a social group as like an organism, whose members work together to benefit the common good. Another theme is centered on the concept of altruism, which is selectively disadvantageous within groups and therefore requires betweengroup selection to evolve. These two themes sound similar and compatible, but in fact there are important differences. For example, in models of altruism, the most adaptive group is one that contains $100 \%$ altruists. In contrast, the parts of an organism are differentiated into organs and cell types. As another example, highly altruistic traits require strong group selection to evolve, to counteract strong selection within groups. But dividing labor, organ-like, need not be self-sacrificial at all.

I applaud Smaldino for advancing the "group as organism" theme in his target article. In this commentary, I will argue that his points apply to all multilevel evolutionary processes, not just human cultural evolution.

It is helpful to begin with the familiar case of individuals as the unit of selection in genetic evolution. Momentarily forget about group selection or genomic conflict and imagine a species in which natural selection acts entirely at the individual level. Organisms are selected on the basis of their phenotypic properties, which are mechanistically caused by genes and their interactions. Occasionally a single phenotypic trait can be attributed to a single gene, but most often the phenotype-genotype relationship is more complex, with single phenotypic traits caused by many genes and their interactions. Moreover, when the same phenotypic trait is selected in different populations, the response to selection often involves different genes. As an example, for adults, the ability to digest lactose has evolved at least twice in human populations, but different mutations were selected in each case (Holden \& Mace 2009). Given the chance nature of mutations, it is unlikely that the same one would arise in different populations subjected to the same selection pressure.

In short, when individuals are the units of selection, they also become the unit of functional analysis. The elements that comprise the unit are studied as proximate mechanisms. This is often simpler in principle than in practice, because the proximate mechanisms can be so complex, involving gene-gene and geneenvironment interactions operating throughout development. In fact, some authors would fault my description as too genecentric, when genes are merely parts of self-replicating developmental systems (e.g., Oyama et al. 2003).

The complexity and distributed nature of the proximate mechanisms is a severe barrier to understanding. We cling to singlelocus models and the idea that phenotypic traits are caused directly by genes to avoid the complexity, but this is at our peril because real-world complex systems have properties that our imaginary simple systems do not.

Frame-shifting upward, when groups become the unit of selection, they also need to become the unit of functional analysis. Individuals and their interactions must be studied as proximate mechanisms, similar to genes within organisms. Sometimes the relationship between a group-level phenotype and the actions of individuals will be simple, but often it will be complex and distributed. Sometimes individuals will be aware of the role that they are playing, but often they will not. For anyone accustomed to thinking of individual organisms as units of functional analysis, it can be unsettling to think of them as proximate mechanisms; however, that is precisely what needs to be done and what Smaldino is arguing for.

Smaldino orients his discussion toward human cultural evolution, but most of his major points apply with equal force to any multilevel evolutionary process, regardless of the inheritance mechanism. It is fascinating to compare the theoretical literature on multilevel selection with artificial selection experiments at the group level. The theoretical models make simplifying assumptions, such as phenotypic traits coded by genes at single loci. Those assumptions result in certain conclusions, such as phenotypic variation among groups declining with the number of individuals colonizing the groups. When real groups are created in the 
Commentary/Smaldino: The cultural evolution of emergent group-level traits

laboratory, however, phenotypic variation among groups remains high even with large numbers of initial colonists. And when groups are selected on the basis of their phenotypic properties, there is typically a response to selection (Goodnight \& Stevens 1997). The theoretical models with their simplifying assumptions got it wrong. Theoretical models that assume more complex interactions do a better job (e.g., Bijma \& Wade 2008; Gilpin 1975; Goodnight 2011; Wilson 1992).

These conclusions apply to social insect colonies, as well as to other groups. One of my few disagreements with Smaldino is that he endorses the formulaic statement that eusociality in insects can be explained by high genetic relatedness or as "the extended phenotype" of the queen. High genetic relatedness contributes to heritable phenotypic variation among groups, but the colony is the unit of selection, and the proximate mechanisms that evolve are highly distributed among individuals. It is hard to imagine the cavity selection process of honeybee swarms as the extended phenotype of the queen when the queen plays no role whatsoever (Seeley 2010).

I do agree with Smaldino's points about equivalence. It is important to establish the equivalence of multilevel selection theory and inclusive fitness theory for models of individual-level traits, so that they are not pointlessly argued against each other. The more individuals become part of a complex distributed system with a group-level adaptive function, however, the more difficult it becomes to imagine them as optimizing units.

To summarize, whenever selection operates at a given level of a multitier hierarchy, units at that level should become the object of functional analysis, and units at lower levels should be studied as proximate mechanisms. This intuition already exists for the study of genes in individuals, when individuals are the unit of selection. It is only beginning to be applied for the study of individuals in groups, when groups are the unit of selection. Smaldino's target article is an important step in this direction with an emphasis on human cultural evolution, but the same algorithm applies to all multilevel evolutionary processes.

\section{Many important group-level traits are institutions}

\section{doi:10.1017/S0140525X13003087}

\section{Matthew R. Zefferman ${ }^{\mathrm{a}}$ and Peter J. Richerson ${ }^{\mathrm{b}}$ \\ ${ }^{a}$ Graduate Group in Ecology, University of California, Davis, Davis, CA 95616; ${ }^{\mathrm{b}}$ Department of Environmental Science and Policy, University of California, Davis, Davis, CA 95616. \\ mrz1@nimbios.org \\ http://www.zefferman.com \\ pjricherson@ucdavis.edu \\ http://www.des.ucdavis.edu/faculty/Richerson/Richerson.htm}

\begin{abstract}
Smaldino makes a solid contribution to the literature on the evolution of human social organization by pointing out that group-leveltraits (GLTs) often emerge from the interactions of group members in such a way that their effects are not easily partitioned into individual selection. However, we argue that he too readily dismisses institutional analysis as a tool for understanding these traits.
\end{abstract}

We largely support the main argument of Smaldino's article and think his ideas about GLTs will be useful for understanding the evolution and origin of complex organizations and societies. We especially welcome the insight that many important GLTs are emergent properties that may be difficult, or even impossible, to partition into units of individual selection. However, we also think Smaldino is too quick to dismiss existing institutional frameworks as useful ways to think about GLTs.

In multilevel selection (MLS) models of cultural evolution, the fitness consequences of traits are partitioned between at least two components, typically group-level and individual-level components (Henrich 2004a). For example, if groups of agents play public goods games, the group benefits of the public good may be accounted for in between-group fitness, and the individual costs may be accounted for in within-group fitness. The typical argument against MLS accounting is that partitioning at the group level can be unnecessary because selection can often be more parsimoniously accounted for at the individual level. However, Smaldino takes the opposite track, arguing that group traits are emergent and, by definition, not easily reduced to aggregates of individual-level traits.

Although we agree with the broad strokes of Smaldino's thesis, we think he errs in denying that many of these GLTs can be usefully classified as "institutions" and studied using existing tools of institutional analysis (e.g., North 1990). Institutions are often defined as the "rules" that are the properties of groups called "organizations." Institutions range from informal norm-based rules to formally codified laws of complex societies, and a key feature of institutions is that they are often maintained by systems of rewards and punishments that suppress individuallevel deviation and stabilize behavior at a game theoretic equilibrium. Because the space of stable institutions is vast in both theory and in practice, institutional change can often be appropriately modeled as between-group "equilibrium selection" (Boyd \& Richerson 1990). In fact, it is relatively common for institutional economists and political scientists to treat formal institutions, such as the rules of political and economic organizations, as group-level traits in direct evolutionary competition. This is the sort of simplification to group-level traits for which Smaldino seems to advocate.

"Constitutional democracy" is a good example of an institutional GLT that can spread between countries as a result of selectionlike processes (Cederman \& Gleditsch 2004). However, breaking something as complicated as the rules governing a constitutional democracy down to units of individual-level selection seems prohibitively difficult - a constitutional democracy is more than just the aggregate sum of individual-level costs and benefits. In short, it is emergent.

Smaldino gives three examples of where he does not consider institutions to qualify as group level traits:

Group-level traits are related, but not equivalent to institutions... a group-level trait is the phenotypic effect of social organization. Thus, examples of group-level traits are the music rather than the rock band, the election of a leader who reflects the public interest rather than the democratic voting system, the sailing ship's voyage rather than the crew positions, the economic surplus rather than the market economy. (sect. 2, para. 2)

We disagree with Smaldino and think that an institutional framework is more useful for understanding the spread of GLTs than a focus on phenotypic effect.

Taking Smaldino's suggestion that a GLT should be considered the "economic surplus rather than the market economy" (sect. 2, para. 2), one might ask how does economic surplus spread? Does a poorer country look to a richer country and say "we should copy their economic surplus?" That probably would not get them very far. Instead, a country might more successfully emulate the economic or political institutions that may underlie another country's economic surplus. We might therefore think of "economic surplus" as an indication of the success of the GLT, not the trait itself.

Taking Smaldino's other examples; a country is more likely to emulate a successful country's political institutions than it is to elect their political leaders. Similarly, during the Meiji restoration, the Japanese slavishly emulated British naval institutions, including standard crew positions (Goldman 2002). However, they did not attempt to recreate particularly successful British voyages.

Although the institutional framework is more useful than Smaldino supposes, not all GLTs need be institutions. A lot of bands emulated the music of the Beatles, for example. However, although the sound of a Beatles' song can be emulated directly, 
Response/Smaldino: The cultural evolution of emergent group-level traits

it is most efficient to do it with the same numbers and types of musicians. Some styles of music have an institution-like structure enforced by fans. Fans can usually tell you their favorite style of music-classical, country and western, folk, rhythm and blues, rock, etc. Musicians who change styles may lose all or part of their audience when they do so.

In summary, we think Smaldino's article highlights a future direction for modeling the evolution of social complexity and institutional selection. He convincing argues that GLTs are useful simplifying concepts. We hope his insights will be integrated into the study of both simple and complex emergent institutions.

\section{Author's Response}

\section{Group-level traits emerge}

doi:10.1017/S0140525X13003531

\section{Paul E. Smaldino}

Center for Advanced Modeling in the Social, Behavioral, and Health Sciences, Johns Hopkins University, Baltimore, MD 21209.

paul.smaldino@gmail.com

http://www.smaldino.com

Abstract: Most commentators supported the thesis of the target article, though there were also those who were less fully persuaded. I will begin with a response to the most critical commentaries. First, I will justify an evolutionary perspective that includes group organization and nongenetic inheritance. Next, I will discuss the concept of emergence. Following that, I will transition to an exploration of ideas and concerns brought up by some of the more supportive commentators. This will include a discussion of different types of groups; the psychology of group-level traits; the uses and limitations of an institutional perspective; the link between transmission, adaptation, and selection; current and future methodologies; and the variety of fields that may benefit from a group-level traits perspective.

I am grateful to all the commentators and humbled by the variety of responses and the care that went into them. Thankfully, the majority of commentators are on board with the basic thesis of the target article: that group-level traits are distinct from individual-level traits and constitute different units of analysis in evolutionary thinking. As such, a large part of this response will entail clarifying and extending the idea of group-level traits in response to various suggestions and concerns brought up by the commentators.

Although most commentators supported the thesis of the article, there were also those who were not so readily persuaded that any new perspectives or methods are necessary to understand the evolution of human social complexity. Some of these commentaries expressed concerns over ideas that were at the foundation of my argument. I believe it is crucial for any discussion to have a firm foundation. Therefore, I will begin with a response to some of the most critical commentaries. First, I will justify an evolutionary perspective that includes group organization and nongenetic inheritance. Next, I will discuss the concept of emergence and how I use it to elucidate group-level properties and behaviors.

Following that, I will transition to an exploration of a number of important ideas and concerns brought up by some of my more supportive commentators, as well as by those who accept the basic thesis but were critical of some of the details. This will include a discussion of differences among types of groups; the psychology of group-level traits; the uses and limitations of an institutional perspective; the link between transmission, adaptation, and selection in the evolution of group-level traits; current and future methodologies for the study of social behavior and evolution; and the various fields in the biological and social sciences that may benefit from a group-level traits perspective.

\section{R1. The false allure of "standard evolutionary theory"}

The most severe critique of the target article comes from Scott-Phillips \& Dickins, who claim there is no need whatsoever for new methods for understanding grouplevel traits. Their attack is not, in fact, leveled explicitly at the group-level traits idea but is a larger attack on recent advances in evolutionary theory that incorporate methods of nongenetic inheritance; these include cultural evolution, epigenetic inheritance, and niche construction. They argue that these approaches are misguided and represent disruptive deviations from the path to an enlightened understanding of evolution, one that focuses on those methods that they view as constituting "standard evolutionary theory." As this is a critique on the overarching framework essential to the approach presented in my target article, it is important to address their comments first.

Scott-Phillips \& Dickins's critique stems from two beliefs: (1) that all heritable traits, including behaviors, are derived from genes (e.g., Dickins \& Rahman 2012), and (2) that culture is a strictly proximate mechanism. Neither belief withstands closer examination. First, a gene-only view of evolution ignores the role of self-organizing forces in shaping traits (Kauffman 1993) and the fact that nongenetic phenotypic factors may be both heritable and subject to natural selection, including epigenetic markers (Jablonka \& Lamb 2005), environmental niches (Odling-Smee et al. 2003), and cultural institutions (Richerson \& Boyd 2005). Second, treating culture as a proximate mechanism ignores both the coevolutionary interaction between culture and genes and the fact that culture itself evolves in a Darwinian process (Mesoudi 2011). Scott-Phillips \& Dickins also seem confused about basic principles of multilevel selection theory. For example, they argue against the use of multilevel selection (MLS) theory by saying "there is no formal justification to consider the cultural group as a unit of selection." But MLS theory does not, in fact, require that the group is a unit of selection, only that the circumstance of belonging to a group influences individual fitness (Okasha 2006). Finally, Scott-Phillips \& Dickins take me to task for ignoring the work of cultural epidemiologists such as Dan Sperber. I agree that cultural epidemiological models are valuable, but their direct relevance to a conceptual discussion of group-level traits is minimal. Further, the cultural evolutionary perspective promoted by Boyd, Richerson, and colleagues (Boyd \& Richerson 1985; Richerson \& Boyd 2005; Mesoudi 2011) is completely coherent with cultural epidemiological models (Henrich et al. 2008), and because these models also treat cultural variants as individual-level traits, the discussion in the target article applies to them as well. 
Response/Smaldino: The cultural evolution of emergent group-level traits

Evolutionary theory is a relatively young field, one that continues to develop as researchers identify new principles and incorporate more of the real world's daunting complexity into their models. It is, of course, important to study established methods and frameworks, and to work within them when appropriate to avoid reinventing the wheel. But it is not pragmatic to cling to a restricted body of theory deemed "standard" while ignoring important advances that will help us to better understand biological evolution, as well the processes of change in other systems, such as human culture. The issue here seems to be largely a problem of perspective. Sometimes adopting different perspectives, metaphors, or frameworks helps us to visualize a system, and related problems, in different ways. As Kauffman (1971) eloquently elucidates, there is often not a single "correct" model for conceptualizing a complex system. Rather, the model one adopts - and the subsequent articulation of the parts of the systemdepends on the particular questions one asks. The genecentric perspective of "standard evolutionary theory" is not wrong, but insisting on it as the only valid model limits one's vision, and thereby limits the questions one can ask, let alone answer.

\section{R2. On emergence}

Emergence is a tricky concept, and one that has long been the subject of debate among complexity theorists and philosophers of science. Okasha notes that the emergent/ aggregate distinction is often imprecise. I agree, which is why in the target article I note that the degree of emergence I discuss must often be determined heuristically rather than algorithmically, and I suggest using Wimsatt's (1997; 2006) heuristics for aggregativity to do so. A system that fails to exhibit all four of Wimsatt's aggregate properties exhibits the type of emergence I wish to discuss. Schank makes the useful suggestion of distinguishing between "strong" group-level traits that meet this criterion and "weak" group-level traits (such as flocking) that are aggregate to some greater degree. For convenience I will use the term "group-level traits" to indicate the strong subtype throughout this response.

Santana \& Weisberg misinterpret me as claiming that strong group-level traits are necessarily irreducible. This is understandable, as that is indeed what some authors have meant by invoking the term "emergence." Santana \& Weisberg propose that group-level phenomena are always reducible, and can be captured by agent-based models in which only the actors and their relationships are represented. I am quite enthusiastic about the potential for agent-based modeling to address the problems I discuss in the target article, but I disagree that explicit representation of group-level phenomena is unnecessary. To untangle these issues, it will be helpful for me to clarify my position on emergence.

Some authors have proposed that "emergence" refers to a category of phenomena that can be rigorously defined, and therefore is a natural kind in the sense that it exists in the universe independent of an observer (e.g., Bedau 2008). It is not my intention to enter into a discussion of the metaphysical realness of emergent phenomena. A phenomenon is emergent, in the sense I am using the term, when the language of more primitive concepts and relationships fails to capture the phenomenon at hand. Whether or not the emergent phenomenon is a natural kind, there are still real patterns at higher levels of organization to be recognized by an observer, patterns that may lead to improved descriptive parsimony and/or enhanced predictive power over lower-level descriptions (Dennett 1991). For example, the precise mechanical behavior of a honeybee colony is of course reducible to all the individual bees, their environments, and their relationships. However, if we are to speak of the colony solving problems, such as when a swarm successfully chooses the optimal site for its nest through a democratic process of exploration and presentation (Seeley 2010), then we can properly speak of emergent behavior, because the individual bees do not solve the problem; only the colony as a whole does that. Similarly, the patterns of activity in the human brain are reducible to the individual neurons, glial cells, and their physical environments, but conscious attention and memory are emergent, because these terms describe the behavior of a higher-order entity beyond that of a collection of individual neurons (Hofstadter 2007). This is also, perhaps, why efforts to reduce all of the natural and social sciences to physics are so unsatisfactory (Fodor 1974). Economics does not reduce to physics, even though all the entities involved are surely rooted in physical phenomena. This is because the entities of economics only make sense (to the observers describing them) in terms of relationships between actors, institutions, markets, and capital. These entities are, in turn, psychological and sociological in nature, and are not appropriately described by invoking a (vast) set of physical relationships for the simple reason that they only have meaning as higherorder constructs.

Strong group-level traits are emergent in this sense. Admittedly, things may get semantically hairy, because we can sometimes speak of the behavior of a collective even when that behavior is highly flexible to the organization of the individual constituents. I agree with Ibbotson when he cautions that one must make sure to rule out individual-level explanations for social phenomena before looking to the group level. When a group-level analysis is appropriate, it is valuable to adopt Wimsatt's heuristics and focus on strong group-level traits.

Understanding groups as entities capable of emergent properties is also important because a group's organizational structure and social resonance can exert influence back onto its constituents, a phenomenon known as downward causation (Campbell 1974a). Higher-level entities (groups) affect lower-level entities (individuals) through proximate psychological and sociological mechanisms, as well as via selective forces that promote or disrupt the individual-level features that facilitate particular group structures. When the mechanisms of downward causation are proximate, group-level traits may indeed be irreducible, because the group's constituent actors are influenced by higher-order constructs. For example, a team of athletes, hunters, or soldiers may be particularly effective as a result of synergistic interactions between teammates. This effectiveness creates a reputation that influences the behaviors of the team's constituents, as well as the perceptions and behaviors of the individuals and groups with whom the team interacts. In such cases it may still be possible to capture group-level phenomena with an agent-based model, as Santana \& Weisberg suggest, but only if 
Response/Smaldino: The cultural evolution of emergent group-level traits

group-level traits are explicitly expressed in the model as potential causal factors (see Schank 2001). This relates to my discussion in the target article concerning interactional complexity. When it comes to influences between levels of organization, there is not one causal arrow but a quiverful, pointing every which way.

\section{R3. On groups and their constituents}

The question "what is a group?" is complicated by the fact that there may be many different kinds of groups, each with different organizations, functions, and ontogenies. Further, the human psychology behind complex group organization is far from simple. In this section, I address the concerns related to group type, the psychology of group-level traits, and the cultural milieu that produces group-level organization.

\section{R3.1. The varieties of group experience}

In the target article, I concentrated on describing the group-level trait as something categorically and qualitatively distinct from an individual-level trait. To do this in the broadest possible sense, I adapted Wilson's (1975) definition of the trait group, which led to the inclusion of everything from mother-infant relationships to the interactions of a vast army under the same categorical heading. Highlighting the group-level trait as an overarching category is important. However, I also agree with Fuentes that dyadic and large-scale interactions should not be assumed to be functionally equivalent entities in an evolutionary model. For more precise analysis, a better taxonomy of groups - and group-level traits - may be useful.

Gerkey \& Cronk correctly point out that there are many types of groups, and they suggest several ways of distinguishing them. I am most drawn to their distinction between "corporate" and "categorical" groups, as the former type seems most likely to exhibit emergent (strong) group-level traits, while the latter is the type most commonly referred to in cMLS models. In general, Gerkey \& Cronk's designations of group types, which are based on a study of real human groups and social networks, strike me as more useful than the more traditional distinction in MLS theory between interdemic and intrademic group selection (Boyd \& Richerson 1985; Wade 1978). It is possible that there may even be systematic differences in the types of organizational structures - and hence the types of group-level traits - that are prevalent or even possible in each of Gerkey \& Cronk's suggested group types, and that cultural evolution may operate differently on each.

Caporael \& Garvey take a slightly different approach, proposing a model of "core configurations," in which different "core" group sizes point to different types of organizational structure and are associated with different types of tasks. In this case, group type is inexorably linked with group size. Caporael \& Garvey suggest that group size at each of their four proposed core configurations (dyad, task group, deme, macrodeme) corresponds to different types of group tasks and therefore different key grouplevel traits. There appears to be a good deal of validity to this approach. What is most important about this framework is that it links group-level traits with both individual psychology and the evolution at the population level of traits related to social coordination. More will be said on both those topics in subsequent sections of this response.

\section{R3.2. The psychology of group-level traits}

Human groups exhibit and transmit group-level traits in a manner unique in the animal kingdom. This fact demands an inquiry into the unique features of human psychology that make such an organizational feat possible. I therefore agree with Muthukrishna \& Schaller that understanding the proximate processes involved in group coordination and the emergence of group-level traits is essential. They are right to highlight the small but steadily growing trend in social psychology to embrace dynamical systems theory (Vallacher \& Nowak 2007), which seems like as good a framework as any with which to study the psychology of group-level traits. In this section I will briefly discuss several avenues of psychological research that I (along with several commentators) believe are particularly pertinent to the study of group-level traits.

As noted by Caporael \& Garvey, humans may possess different mental systems for dealing with different types of groups, organized by size and functionality. However, it is also important not to be too quick to assume the presence of specialized mental functions. The environment, including the social environment, shapes mental processes and creates affordances that constrain choice and guide behavior (Gibson 1979; Smaldino \& Richerson 2012). Even reliably emerging features of social interactions may be the result of affordances from underlying social structure rather than reflections of explicit psychological hardwiring.

Theiner \& Sutton focus on group problem-solving and distributed cognition, in which individuals collaborate to solve problems in complex ways. I agree with them that it is vital not to compare too directly group performance on a task with individual performance, as the goals and payoffs may not be the same. I must admit that I found their presentation of distributed cognition somewhat vague. A potentially clarifying idea was recently presented by Gallotti and Frith (2013), who propose that collaborating minds engage in an irreducibly collective mode called the "we-mode." An example is that the presence of a known collaborator introduces new affordances for the decision maker. Imagine that an important object is out of reach for me, but not for my teammate or social partner. I can make collective plans that involve the object, because even though I cannot reach it, we can. The "we-mode" seems crucial for many (though not all) group-level traits.

A number of previously identified psychological features may have importance for the study of group-level traits, and vice versa. Two mentioned by O'Gorman are transactive memory (Liang et al. 1995) and the immense human propensity for group identity. With regard to group identity, Roccas and Brewer (2002) have noted that individuals often identify with several groups simultaneously. These group identities can be hierarchically nested or practically orthogonal, and circumstance can evoke stronger associations with one group over another. This ability to identify with multiple groups is likely important for the facilitation of group-level traits, as individuals can participate in a number of distinct group endeavors and feel strong ties to each organizational unit. Abrams posits that not only do social roles facilitate group-level organization, but also 
that "different social roles may encourage 'cohesive' cognitive subnetworks, reinforcing particular patterns of thought in individuals who fill similar social roles." This suggests that the environmental effect of participating in a grouplevel endeavor may lead to a number of cognitive and behavioral similarities among individuals occupying similar social roles, above and beyond those necessary for performing those roles. A related phenomenon is that persistent influence (e.g., via physical proximity) can evoke synchrony in systems that exhibit periodicity, such as neurons and other oscillators (Strogatz \& Stewart 1993). It seems possible that more complex psychological functions could work similarly, at least on some level. I find this idea quite intriguing.

A psychology that facilitates group cohesion is one prone to respond to and adopt social norms, and there is good evidence that humans possess such a psychology (Chudek \& Henrich 2011; O'Gorman et al. 2008b). Nevertheless, Schank rightly points out that imitation is not a sufficient psychological mechanism for learning, because imitating another's practices also involves the selection of which practices are (partially) imitated. This is related to the more general problems involved in the psychology of option generation, reviewed in Smaldino \& Richerson (2012).

MacDonald accuses me of having an "inadequate psychology of groups." In the target article, I chose to focus on defining the concept of the group-level trait at the organizational level and discussing its role in cultural evolution. I completely agree that understanding the psychology of group-level traits is crucial, and I quite explicitly discuss how human psychology has been shaped to deal with group living, including mechanisms that fall under what MacDonald describes as "explicit processing" (e.g., leadership, teaching). For example, I discuss the importance of pedagogy for the development of social roles, as well as Durham's (1991) concept of TRIMs, which are the "ecological, psychological, linguistic, and cultural barriers to the blending of cultures." I do agree with MacDonald, however, that my discussion of human psychology is somewhat cursory. I felt that there was only so much material I could cover without overly tangling the narrative thread.

MacDonald also misrepresents my position on behavioral genetics when he says that I "exclude by fiat the behavior genetic literature." My point about repeated assembly is to contrast it with views of behavioral genetics that imply that genetic influences to behavior can be considered additive or otherwise simplistic. Genes and environments interact through a process of continuous feedback. I completely agree that genetically influenced individual differences, such as differences in personality, should be related to theories of the emergence of division of labor. Recent advances by evolutionary researchers on the emergence and stability of individual differences (e.g., Johnstone \& Manica 2011; McNamara \& Leimar 2010; Wolf \& McNamara 2013) will be an exciting avenue to incorporate into the study of group-level traits.

\section{R3.3. Culture is more than individual differences}

Culture is not simply a statistical description of individual differences, contra Kim, Jeong, \& Park (Kim et al.). They criticize my position that culture influences cognition and claim that I characterize culture as an "autonomous, external variable that independently affects cognitive processing." I want to be clear: I think no such thing. Rather, I think that what the cultural psychology literature shows quite clearly is (1) how much our thinking, perception, and behavior are influenced by learning, especially the learning that occurs during development, and (2) how cultural groups provide a common ecological and social environment that leads to highly correlated patterns of cognition among members of the same culture. Kim et al. claim that "culture is an aggregate of individual differences in psychological variables within and between groups." This claim is not supported by the overwhelming evidence that individuals tend to adopt the perceptual and behavioral norms of the culture of their birth, independent of their genetics (Boyd et al. 2011; Cohen 2001; Harris 2012; Hespos \& Spelke 2004; Kelly et al. 2007; Kinzler et al. 2009; Kuhl et al. 1992). This is not to say that there are not regional or cultural regularities in genes, or that individuals do not differ in temperament, only that cultural (e.g., socially learned) factors are also tremendously important. Moreover, cultures are defined by much more than the aggregate psychologies of their members. Cultures also involve traditions of learning and customs, of institutions and infrastructure. The fact that bride theft is prevalent in Kyrgyzstan and not in the United States (Werner 2009 ) is the result of cultural history, not innate psychological differences. Similarly, the culture of honor still prevalent in the American South and the biological responses that support it (Nisbett \& Cohen 1996) cannot be explained by genetic differences in psychological temperaments, but only by examining cultural traditions. Finally, to address group-level traits, I completely agree that individual differences in innate psychology will influence the opportunities and affordances for an individual's roles in group-level traits. However, it is ludicrous to explain the sailing of a ship or the rituals of a religion solely through an appeal to individual psychological differences. The emergence of such traits depends also on the cultural infrastructure that guides learning and opportunity.

\section{R4. The importance and limitations of institutional analysis}

In the target article, I state that group-level traits are related, but not equivalent, to institutions. I agree with Zefferman \& Richerson that an institutional perspective is important, and it is possible that I downplayed that importance. Institutions - the formal and informal rules that govern social behavior - may often be the source of group-level traits. Individuals in a group may copy another group's institutions in order to absorb a suite of norms and behaviors, as in the example of the Japanese emulation of British naval institutions. Zefferman \& Richerson suggest modeling institutional evolution as a type of between-group "equilibrium selection," as in Boyd and Richerson (1990). Although this is a useful model for some systems, "equilibrium selection" is an interdemic process that does not account for the kinds of grouplevel traits that compete within small groups, in which many different organizational patterns may coexist. Perhaps more importantly, some group-level traits may be the product of many interacting institutions. For 
example, consider the hypothetical Roman Legion I discussed in the main text. This will also help to clarify a question posed by Panchanathan, Mathew, \& Perreault (Panchanathan et al.), who wondered "why a Roman Legion represents a group-level trait, while an insect colony does not." The Legion itself is not a trait, of course. Rather, the organizational properties of the Legion and its constituents lead it to possess a number of traits. An example of a rather superficial trait might be the Legion's skill in battle that leads to them being almost undefeatable on an open plain but much more vulnerable in the close quarters of a dense forest. This trait does not stem from one institution, but from many institutions interacting with the culturally influenced psychologies of the Legion's constituents. Other example traits might include the economic resilience of a firm or the evocative tonality of a musical group.

Because not all group-level traits reduce to institutions, I feel I must reject Waring \& Goff's suggestion to focus on the social organization itself and not on the phenotypic traits produced by that organization. In practice, the organization is the proximate cause of a given group-level trait and will likely be vital to its understanding. However, an explanatory trait may require a different description than that of the organization that gives rise to it. In terms of evolutionary dynamics, fitness is a property of traits, not of individual organisms or groups, and it is not explanatory to speak of the fitness of an organizational structure without appealing to the subsequent trait produced by it. The same is true of more direct influences on behavior. As an example, consider individual-level traits such as "an aggressive personality" or "being particularly gassy." Explanations of their influences on behavior are most meaningfully detailed by invoking the traits themselves, and not through an appeal to neurophysiology or digestive system malfunction.

MacDonald claims that culture is shaped primarily by leaders in a top-down fashion, and is therefore not emergent. In this sense, he argues for the supremacy of explicit institutions enforced through leadership. I do not dispute the importance of leaders, but it seems reductionistic to an absurd degree to suggest that the coordination involved in group activities from sailing a ship to hunting a stag to the intricate dance rituals of numerous religious groups all boil down to leadership. Many processes of social coordination are not dictated by a leader, but emerge organically both through communication between individuals and from the constraints to affordances provided by group structure.

Read agrees that systems of organization that incorporate differentiated roles are important, but claims that "these systems of organization need not be emergent, but are often cultural constructions, such as the culturally formed kinship systems that provide structure and organization in human societies, especially in the small-scale societies that were the evolutionary precursors of largescale human societies." I think that what Read calls "cultural idea systems" are very similar to institutions. However, while institutions are generally classified as sets of rules, "idea systems" will also entail deeply entrenched cultural factors such as language, mythologies, and narratives that structure interactions in more subtle ways and are maintained through group-level organization. Cultural kinship relations represent a fascinating subset of grouplevel traits, and although I agree with Read that these idea systems are of enormous importance to the evolution of human social complexity, I do not agree that they are not emergent. Culture does not exist as an external, top-down force. Rather, culture is repeatedly assembled and emerges from the complex interplay between human psychology, social organization, and history.

In my target article, I focused on the institutional role of religion in enforcing and transmitting suites of social norms. I believe that it is often useful to regard religion as an institution (or set of institutions). Nevertheless, I am also in agreement with Sosis \& Kiper's suggestion that a religion is much more than its institutions and is more fully described as a complex system. This is especially true when considering the evolution of religion within a population - not the fast transmission of a conversion or a conquest, but the slow change that occurs as beliefs, rituals, and institutions adapt to internal selection pressures. As Sosis \& Kiper point out, religions do not simply exercise top-down control over norms, but rather permeate all aspects of social life, coevolving with the very norms they prescribe and proscribe. The complex adaptive systems perspective advocated by Sosis \& Kiper is not only useful, but is probably necessary for gaining a better understanding of the complicated dynamics of group-level traits in terms of their function, their proximate causes and effects, and their evolution.

\section{R5. Transmission, selection, and adaptation}

The major idea proposed in the target article was that group-level traits are an often-unconsidered factor in human evolution. Group-level traits influence the evolutionary trajectory of individual phenotypes and also evolve in their own right. The time frame of a group-level trait does not map one-to-one with the life cycle of an individual human, which creates a problem for evolutionary modeling of group-level organization. Several commentators raised important issues with the transmission, selection, and adaptation of group-level traits. In this section, I will attempt to address these concerns.

\section{R5.1. How are group-level traits transmitted?}

The transmission of social roles-and therefore the complex social structure that facilitates many group-level traits - is repeatedly assembled in a process of feedback and structuring. I was grateful to see that several commentators explicitly agreed with me on this point (Caporael \& Garvey; Waring \& Goff; Schank). I also agree with Read that an important challenge is to understand the phylogeny of this ability that, while perhaps not completely unique in the animal kingdom (O'Gorman; Wilson), is certainly hypertrophied in humans.

The transmission of group-level traits is a complex issue, and my preliminary discussion admittedly only scratches the surface. Davis \& Margolis believe that my characterization of group-level traits is problematic, because "the basic aim of evolutionary theories based on population modeling"-including cMLS approaches - "is to explain frequencies in populations. Yet it makes no sense to ask, for a given group, about the frequencies of its group-level properties" (paras. 2 and 3). However, this is not a problem for my presentation, but rather a serious problem for 
evolutionary theorists who acknowledge the existence and importance of group-level traits, as Davis \& Margolis appear to do. Classic population models are indeed based on frequencies of traits; these methods are likely insufficient to explain the evolution of group-level traits. Without even getting into emergent group behavior, simple frequency-based models have already been shown to fail when complex social or spatial organization is paramount (Goodnight et al. 2008; Smaldino et al. 2013b). Emergent group-level traits may require new methods, as I will discuss further below.

Davis \& Margolis are also skeptical that transmission of group-level traits requires group-level explanations. If a singer wants to form a band that sounds like the Beatles, they suggest, he can separately seek out a drummer who plays like Ringo and a bassist who plays like Paul. Although such a strategy could perhaps yield a serviceable band, bass/ drummer synchrony in rock bands is paramount to the sound of the music. The bassist must be able to play "in the pocket" of the drummer's beat. Rock bands that sound good often do so because the styles of the constituent musicians click, which is why "supergroups" made of undeniably talented musicians from different successful bands often disappoint. Moreover, sounding like the Beatles is very different from being influential like the Beatles, or having audiences like the Beatles. These traits likely require group-level explanations.

The recipient of individual-level knowledge-information held in individual brains - must be an individual. However, Davis \& Margolis err in claiming that social transmission must occur at the level of individuals. Group-level organization can emerge through processes involving the interplay of individual decisions, social affordances, and repeated assembly. Even when an organizational plan is contained within the mind of one individual, it may be the case that other participants do not possess that knowledge, and that it is only a small piece of knowledge related to skill or social roles that is transmitted to an individual. Moreover, the transmission of the social infrastructure for group behaviors - for example, religious rituals, multiperson sailing voyages, coordinated cooking - may be akin to an $n$-person Stag Hunt game (Pacheco et al. 2009) in that a critical threshold of individuals must learn their correct social roles in order for the trait to persist.

\section{R5.2. On the units of selection}

The phrase "unit of selection" is a loaded one, and one I half regret using in the target article because I think it distracts from the larger points made therein. Santana \& Weisberg take issue with my characterization of group-level traits as a unit of selection. I agree that a group-level trait is neither an interactor nor a replicator in the sense described by Hull (1980). However, neither are individual-level traits (such as eye color), since the traits themselves do not replicate. Yet traits are what selection acts upon. Fitness is a property of traits, not organisms or groups (Sober 1984). Just as it makes sense to speak of organisms with certain traits being selected over other organisms with other traits, so it makes sense to speak of patterns of group organization being selected for. Wilson makes this point quite clearly in his commentary.

\section{R5.3. Transmission, selection, and adaptation are entangled}

Panchanathan et al. point out that group-level traits can arise from individuals maximizing utility, and not necessarily from group selection. I agree that it is necessary to separate selection on group-level traits from "group selection." Not all group-level traits require the assumption of between-group competition. Humans evolved in a social milieu (Caporael \& Garvey), and group-level traits should be able to spread through both individual-level and group-level selection. However, it may not be as easy as Panchanathan et al. imply to disentangle selection from adaptation when it comes to group-level traits. This is because trying to understand the evolution of grouplevel traits can lead to what Schank calls "selection-transmission thickets." Schank writes:

Mechanisms for selecting, generating variation, and transmitting cultural traits are often intertwined. For example, imitating the practices of one group also involves the selection of which practices are partially imitated. [...] To this entanglement of mechanisms of selection, variation, and transmission, it must be added that as cultures evolve and become increasingly complex, new vicarious selection, variation, and transmission mechanisms (VSVTMs) evolve and appear at new levels in culture.

Humans have long lives and a rich capacity for cultural (social) learning, as well as for complex social behavior (strong group-level traits), and as such the distinction between selection, variation, and transmission may become blurred. Additive or multiplicative models may therefore be of limited use when trying to understand the evolution of group-level traits.

This is also why the methods championed by Chudek \& Henrich in their commentary are insufficient. I quite agree with them that "making sense of this complex emergent domain" is far from simple. If it were simple, a formal model in a well-established methodology would have been sufficient instead of a lengthy target article to simply introduce the problem. I also agree with Chudek \& Henrich that the culture-gene coevolutionary approaches pioneered by individuals such as Boyd, Richerson, and Henrich represent some of the best attempts so far to understand the dynamics of the evolution of human social complexity. I am not in the least looking to disparage any of that work, and I agree that "starting at the start" is a key approach. However, I do not believe that the options are limited to "starting at the start" or "starting at the end," as Chudek \& Henrich imply. Indeed, studying the evolutionary dynamics surrounding group-level traits represents starting somewhere in the middle, which of course is always the most difficult part to explain in any complex story. A three-minute pop song can capture the start of a romance or its end, but it takes a novel to detail the relationship's messy middle. Similarly, it will take more complex methodologies to capture the evolutionary dynamics of group-level traits. Such approaches will add to, not subtract from, our understanding of cultural evolution.

\section{R6. Models and methods}

The evolutionary dynamics of group-level traits are still poorly understood, and making headway in that understanding represents a significant challenge. Many existing 
methods provide important frameworks, and they should not be ignored. Additionally, new methods and models will assuredly be needed to formalize and better conceptualize the role of group level traits in human social evolution.

I agree with Doebeli \& Simon that formal models are crucial to understanding complex systems and for conceptualizing problems within them. Models can distill a system down to manageable parts and relationships, and allow us to see clearly the presence or absence of possibility in a world obscured by the fog of complexity. Doebeli \& Simon propose that their modeling framework (Simon et al. 2013) is appropriate for the evolutionary study of group-level traits. I agree that their framework has a great deal of value. Although I had not previously examined it in detail, it is strikingly convergent with some of my own recent attempts to model social evolution at multiple levels of selection (Makowsky \& Smaldino 2014; Smaldino et al. 2013a). Nevertheless, things can be become quite complicated when groups are ephemeral, or when individuals can belong simultaneously to multiple groups (Lubell 2013). As Doebeli \& Simon acknowledge, their framework would have to be adapted for dealing with a number of aspects of human culture. These include institutions and behaviors such as marriage, property rights, resource inheritance, alloparenting, social enforcement, and division of labor.

Although a formal mathematical framework provides baseline cases and allows for rigorous analysis, the immense complexity of human social interaction may often be mathematically intractable. Computational techniques such as agent-based modeling may instead be more fruitful (Santana \& Weisberg; Taylor \& Bryson). Individuals can be represented explicitly, and therefore the model can incorporate essential heterogeneity related to sex, age, genotype, and group identity (Epstein 2006). Abrams notes that social network models may provide one way of characterizing certain key differences among social roles by capturing differences in the direction, degree, and nature of what is communicated between agents. Another important direction is the "ecology of games" framework (Lubell 2013), which acknowledges that individuals participate in many social organizations simultaneously, that membership can be temporary, and that co-membership with others may overlap in some but not all arenas. Agent-based modeling has investigated institutions that promote assortment of cooperators under these conditions (Smaldino \& Lubell 2011; 2014), but explicit evolutionary analysis has yet to be done.

Although the development of new models is paramount, we still have much to learn from existing models of social evolution and multilevel selection. Ibbotson provides a cautionary tale when he incorrectly claims that "what is good for the group is also good for the individual." It is clear that this is not always the case. The adoption of a strategy or institutional framework can raise the average fitness in a group while lowering the relative or even the absolute fitness of certain individuals. Traditional multilevel modeling approaches can show how a trait that increases the average group fitness can evolve even when it is detrimental to the individual. Meanwhile, game theoretic constructs must move beyond simple cooperation. McCain helpfully points out that such games exist. He presents a very interesting game that has elements of both coordination and anti-coordination. I agree that this simple game may better represent the kinds of scenarios in which organized cooperation with division of labor works best. I have not seen an evolutionary analysis of such a game, but I agree that such an analysis could be valuable in understanding the emergence of social complexity. Nevertheless, as Mattei notes, the complex psychology of group-level traits presents a challenge for game theoretic modeling. For example, empirical research has shown that individuals' behavior in public goods games is heavily influenced by cultural norms (Gerkey 2013; Henrich et al. 2005), as well as by the opportunity for players to communicate, even when communication is anonymous and therefore cannot influence reputation (Baum et al. 2012).

Nonacs \& Kapheim brought to my attention their very useful concept of social heterosis, which is when individuals carrying each variety of a particular allele have a higher fitness in the presence of individuals carrying different alleles than with individuals carrying like alleles. Grouplevel traits in which individuals must differentiate require by definition a degree of social heterosis. In fact, Henrich and Boyd's (2008) model of division of labor entails a form of cultural social heterosis. When differentiated individuals exhibit a trait by virtue of their differences, Nonacs \& Kapheim's models of social heterosis may be useful for capturing the relevant fitness trade-offs and evolutionary dynamics, and they are quite right that their modeling framework is fully compatible with classic MLS theory. Nevertheless, their model contains neither social organization nor cultural transmission. The benefit of a social trait depends on both the presence of other types of individuals and on a social environment that provides the affordances for displaying the trait. Agent-based and/or network theoretic interpretations of the social heterosis idea that allow for nongenetic (cultural) inheritance will be an important avenue for future research.

Whether our data comes from empirical studies of human genetic or cultural evolution or from a computational model, how can we identify the influence of group-level traits? Okasha suggests contextual analysis, which is a powerful method for establishing the influence of traits that affect group-level fitness, and is in some ways superior to the approach centered on the Price equation that has been more popular in analyses of cultural evolution (Okasha 2006). However, although contextual analysis can test for the presence of group-level effects on selection, it cannot distinguish between weak and strong group-level traits (Schank), nor help us understand how the relevant group-level traits influence the fitness of other individual- and group-level traits. Still, I would love to see contextual analysis, perhaps modified if need be, applied to the study of the evolution of emergent grouplevel traits.

\section{R7. A focus on group-level traits can benefit the study of social behavior in many fields}

In the target article, I focused on the importance of a group-level traits perspective for the understanding of human cultural evolution. As Wilson points out, an appreciation for treating the organized group as a unit of functional analysis is important for evolutionary theory in general, and not restricted to either culture or to humans. Nevertheless, my focus has been on humans. I am certainly not the first to note the importance of social 
References/Smaldino: The cultural evolution of emergent group-level traits

organization for human social behavior, but I agree with Mattei that an explicit evolutionary perspective on those properties and behaviors that are properly defined at the group level can lead to advances in a number of the human sciences, including social neuroscience, game theory, linguistics, and economics, as well as more applied fields like marketing and law.

Shuai \& Gong highlight the study of language evolution, and point out that the language of a group is an emergent group-level trait. Language also clearly facilitates group organization and coordination and is therefore essential in many other group-level traits in humans. Moreover, Shuai \& Gong are right that understanding the roles of complex organization and the competitive advantage of group-level traits is probably crucial for a better understanding of the coevolution of language and human social complexity. As an example, they highlight the importance of language in the formation of social bonds. What else would explain why teenage friends or separated lovers often spend hours on the phone discussing their feelings, their hopes, and their fears? Characterizing human social complexity will probably require more than the simple analyses of static social networks and summary statistics (such as power-law degree distributions) presented by Shuai \& Gong. For example, it is clear that to whom we are tied matters (Granovetter 1973; Hill et al. 2011), as do the dynamics of those ties. Nonetheless, complex models that incorporate social structure with the evolution of language represent a good direction.

\section{R8. Conclusion}

Sitting alone at our desks, it is easy to focus on the aspects of human existence that are best described at the level of the individual. Consider the very process of writing, often characterized as a very solitary activity. I write something. I struggle, alone, and finally manage something I am satisfied with. I send it to the editor. The editor reads it, and gives it his approval. The publisher prints it, and the reader reads it. These are actions with social consequences but individual-level descriptions. Yet this is a false picture of the writing-to-reading process. In reality, I write standing on the shoulders of giants, with the influence of the many articles and books I have read, filtered through my mental models and biases shaped by years of explicit learning and cultural indoctrination. I discuss my piece with friends and colleagues, who shape it further. I submit it to the editor, who solicits the advice of experts, and makes recommendations upon which I base a revision. Upon acceptance, the piece is inspected and refined by a team of proofreaders and editorial assistants. Printers, internet service providers, IT support staff, and mail carriers are all involved in the dissemination of the piece to its readers, who choose whether to read it based on a host of factors of personal history and circumstance, and who interpret it similarly. In the case of the unique structure of this particular journal, a new process starts at this point, as readers become writers, and beget an academic dialogue. The production of this very issue is not something that is produced by any individual, nor is it coordinated by a supreme leader with a host of underlings. Instead, the journal, along with many of its properties, are group-level traits, produced by the complex coordination of organized, differentiated individuals. So too are many, many other aspects of the lives of humans best described.

The role of group-level traits in human evolution is at present poorly understood. Changing that will be a vastly interdisciplinary undertaking, with contributions needed from across those fields concerned with human behavior, evolution, and the dynamics of complex systems. It is an imposing challenge, but one that is surmountable when we work together.

\section{ACKNOWLEDGMENTS}

Thanks to Bert Baumgaertner, Paul Bloom, Lesley Newson, Emily Newton, and Pete Richerson for comments.

\section{References}

[The letters “a” and “ $r$ " before author's initials stand for target article and response references, respectively]

Abrams, M. (2013) A moderate role for cognitive models in agent-based modeling of cultural change. Complex Adaptive Systems Modeling 1(16):1-33. http://www. casmodeling.com/content/1/1/16 [MA]

Aktipis, C. A. (2004) Know when to walk away: Contingent movement and the evolution of cooperation. Journal of Theoretical Biology 231:249-60. [aPES]

Alcorta, C. \& Sosis, R. (2005) Ritual, emotion, and sacred symbols: The evolution of religion as an adaptive complex. Human Nature 16:323-59. [RS]

Alexander, J. M. (2007) The structural evolution of morality. Cambridge University Press. $[\mathrm{MA}]$

Anderson, B. R. O'G. (1991) Imagined communities: Reflections on the origin and spread of nationalism (Revised and extended ed.). Verso. [DG]

Antal, T., Ohtsuki, H., Wakeley, J., Taylor, P. D. \& Nowak, M. A. (2009) Evolution of cooperation by phenotypic similarity. PNAS 106:8597-600. [aPES]

Anton, S. C. \& Snodgrass, J. (2012) Origin and evolution of genus Homo. New Perspectives Current Anthropology 53(6):479-96. [AF]

Aoki, M. (2007) Endogenizing institutions and institutional changes. Journal of Institutional Economics 3:1-31. [aPES]

Apicella, C. L., Marlowe, F. W., Fowler, J. H. \& Christakis, N. A. (2012) Social networks and cooperation in hunter-gatherers. Nature 481:497-501. [DG]
[Dict and the batte for the

Arthur, C. (2012) Digital wars: Apple, Google, Microsoft and the battle for the Internet. Kogan Page Publishers. [DG]

Atran, S. \& Henrich, J. (2010) The evolution of religion: How cognitive by-products, adaptive learning heuristics, ritual displays, and group competition generate deep commitments to prosocial religions. Biological Theory 5(1):18-30. [aPES, MC]

Atran, S. \& D. Medin (2008) The native mind and the cultural construction of nature. MIT Press. [MA]

Atran, S. \& Norenzayan, A. (2004) Religion's evolutionary landscape: Counterintuition, commitment, compassion, communion. Behavioral and Brain Sciences 27(6):713-30. [aPES, TCS-P]

Aumann, R. J. (1959) Acceptable points in general cooperative n-person games. Contributions to the Theory of Games, vol. IV (Annals of Mathematics Studies, Number 40) 4:287-324. [RAM]

Axelrod, R. (1997) The dissemination of culture: A model with local convergence and global polarization. The Journal of Conflict Resolution 41(2): 203-26. [MA]

Axelrod, R., Hammond, R. A. \& Grafen, A. (2004) Altruism via kin-selection strategies that rely on arbitrary tags with which they coevolve. Evolution 58:183338. [aPES]

Baber, C., Smith, P. A., Cross, J., Hunter, J. \& McMaster, R. (2006) Crime scene investigation as distributed cognition. Pragmatics and Cognition 14(2):35785. [GT]

Baldini, R., Beheim, B., Bell, A., Demps, K., Frost, K., Hillis, V., Mathew, S., Newton, E. K., Newson, L., Ross, C., Smaldino, P. E., Waring, T., Zefferman, M. \& Richerson, P. J. (under review). A sketch of the evidence for selection on inter-group cultural variation in humans. [aPES]

Barabási, A. L. (1999) Mean-field theory for scale-free. Physica A 2(72):172-82. [LS]

Barnier, A. J., Sutton, J., Harris, C. B. \& Wilson, R. A. (2008) A conceptual and empirical framework for the social distribution of cognition: The case of memory. Cognitive Systems Research 9(1):33-51. [GT]

Baronchelli, A., Cattuto, C., Loreto, V. \& Puglisi, A. (2009) Complex systems approach to the emergence of language. In: Language, evolution, and the brain ed. J. W. Minett \& W. S-Y. Wang, pp. 141-78. City University of Hong Kong Press. [LS] 
Baronchelli, A., Felici, M., Loreto, V., Caglioti, E. \& Steels, L. (2006) Sharp transition towards shared vocabularies in multi-agent systems. Journal of Statistical Mechanics P06014. Available at: http://iopscience.iop.org/1742-5468/2006/06/ P06014/ [LS]

Barrett, J. L. (2000) Exploring the natural foundations of religion. Trends in Cognitive Sciences 4:29-34. [aPES]

Barrett, L. F. (2006) Are emotions natural kinds? Perspectives on Psychological Science 1:28-58. [aPES]

Barrett, L. F., Lindquist, K. A. \& Gendron, M. (2007) Language as a context for the perception of emotion. Trends in Cognitive Science 11:327-32. [aPES]

Barth, F. (1969) Ethnic groups and boundaries. Little Brown and Company. [DG]

Basden, B. H., Basden, D. R., Bryner, S. \& Thomas III, R. L. (1997) A comparison of group and individual remembering: Does collaboration disrupt retrieval strategies? Journal of Experimental Psychology: Learning, Memory, and Cognition 23(5):1176-89. [GT]

Bateson, P. \& Gluckman, P. (2011) Plasticity, robustness, development and evolution. Cambridge University Press. [AF]

Baum, W. M., Paciotti, B., Richerson, P., Lubell, M. \& McElreath, R. (2012) Cooperation due to cultural norms, not individual reputation. Behavioural Processes 91:90-93. [rPES]

Baumgartner, F. R., Berry, J. M., Hojnacki, M., Kimball, D. C. \& Leech, B. L. (2009) Lobbying and policy change: Who wins, who loses, and why. University of Chicago Press. [DG]

Bedau, M. A. (2008) Is weak emergence just in the mind? Minds \& Machines 18:443-59. [rPES]

Bergmüller, R., Johnstone, R. A., Russell, A. F. \& Bshary, R. (2007) Integrating cooperative breeding into theoretical concepts of cooperation. Behavioral Processes 76:61-72. [aPES]

Bettencourt, L. M. A. (2009) The rules of information aggregation and emergence of collective intelligent behavior. Topics in Cognitive Science 1:598-620. [GT]

Bijma, P. \& Aanen, D. K. (2010) Assortment, Hamilton's rule and multilevel selection. Proceedings of the Royal Society B 277:673-75. [aPES]

Bijma, P. \& Wade, M. J. (2008) The joint effects of kin, multilevel selection and indirect genetic effects on response to selection. Journal of Evolutionary Biology 21(5):1175-88. [DSW]

Bloch, M. \& Sperber, D. (2002) Kinship and evolved psychological dispositions: The mothers' brother controversy reconsidered. Current Anthropology 43(5):72348. [TCS-P]

Boehm, C. (1997) Impact of the human egalitarian syndrome on Darwinian selection mechanics. American Naturalist 150:S100-21. [aPES]

Boomsma, J. J. (2013) Beyond promiscuity: mate-choice commitments in social breeding. Philosophical Transactions of the Royal Society B 368:20120050. [PN]

Bouchard, T. J. \& Loehlin, J. C. (2001) Genes, evolution, and personality. Behavioral Genetics 31:243-73. [aPES]

Bourke, A. F. G. (2011) Principles of social evolution. Oxford University Press. [aPES]

Bowles, S. \& Gintis, H. (2011) A cooperative species: Human reciprocity and its evolution. Princeton University Press. [aPES]

Bowles, S. (2004) Microeconomics, behavior, institutions, and evolution. Oxford University Press. [aPES]

Bowles, S., Choi, J-K. \& Hopfensitz, A. (2003) The co-evolution of individual behaviors and social institutions. Journal of Theoretical Biology 223:135-47. [aPES]

Boyd, R. \& Richerson, P. J. (1985) Culture and the evolutionary process. University of Chicago Press. [arPES, MA, MC, MD]

Boyd, R. \& Richerson, P. J. (1990) Group selection among alternative evolutionarily stable strategies. Journal of Theoretical Biology 145:331-42. [rPES, MRZ]

Boyd, R. \& Richerson, P. J. (1992) Punishment allows the evolution of cooperation (or anything else) in sizable groups. Ethology and Sociobiology 13:171-95. [aPES]

Boyd, R. \& Richerson, P. J. (2002) Group beneficial norms can spread rapidly in a structured population. Journal of Theoretical Biology 215:287-96. [KP]

Boyd, R. \& Richerson, P. J. (2005) The origin and evolution of cultures. Oxford University Press. [aPES]

Boyd, R. \& Richerson, P. J. (2008) Gene-culture coevolution and the evolution of social institutions. In: Better than conscious? Decision making, the human mind, and implications for institutions, ed. C. Engel \& W. Singer, pp. 305-23. MIT Press. [aPES]

Boyd, R. \& Richerson, P. J. (2009a) Culture and the evolution of human cooperation. Philosophical Transactions of the Royal Society B 364:3281-88. [MD]

Boyd, R. \& Richerson, P. J. (2009b) Voting with your feet: Payoff biased migration and the evolution of group beneficial behavior. Journal of Theoretical Biology 257:331-39. [KP]

Boyd, R., Richerson, P. J. \& Henrich, J. (2011) The cultural niche: Why social learning is essential for human adaptation. Proceedings of the National Academy of Sciences 108:10918-25. [arPES]
Boyer, P. \& Petersen, M. B. (2012) The naturalness of (many) social institutions: Evolved cognition as their foundation. Journal of Institutional Economics 8 (1):1-25. [TCS-P]

Brewer, M. B. (2004) Taking the social origins of human nature seriously: Toward a more imperialist social psychology. Personality and Social Psychology Review 8:107-13. [aPES]

Brewer, M. B. \& Caporael, L. R. (2006) Social identity motives in evolutionary perspective. In: Social identities: motivational, emotional, cultural influences, ed. R. Brown \& D. Capozza, pp. 135-52. Psychology Press. [LRC]

Bulbulia, J. \& Sosis, R. (2011) Signaling theory and the evolution of religions. Religion 41(3): 363-88. [RS]

Buss, D. M. \& Greiling, H. (1999) Adaptive individual differences. Journal of Personality 67(2):209-43. [TCS-P]

Buss, D. M., \& Shackelford, T. K. (1997). Human aggression in evolutionary perspective. Clinical Psychology Review 1:605-19. [KM]

Čače, I. \& Bryson, J. J. (2007) Agent based modelling of communication costs: Why information can be free. In: Emergence and evolution of linguistic communication, ed. C. Lyon, C. L. Nehaniv \& A. Cangelosi, pp. 305-22. Springer. [DJT]

Calcott, B. (2008) The other cooperation problem: Generating benefit. Biology and Philosophy 23:179-203. [aPES]

Campbell, D. T. (1956a) Adaptive behavior from random response. Behavioral Science 1:105-10. [JCS]

Campbell, D. T. (1956b) Perception as substitute trial and error. Psychological Review 63:330-42. [JCS]

Campbell, D. T. (1960) Blind variation and selective retention in creative thought as in other knowledge processes. Psychological Review 67:380-400. [JCS]

Campbell, D. T. (1974a) "Downward causation" in hierarchically organised biological systems. In: Studies in the philosophy of biology, ed. F. Ayala \& T. Dobzhansky, pp. 179-86. University of California Press. [rPES]

Campbell, D. T. (1974b) Evolutionary epistemology. In: The philosophy of Karl R. Popper, ed. P. A. Schilpp, pp. 412-63. Open Court. [JCS]

Campbell, D. T. (1974c) Unjustified variation and selective retention in scientific discovery. In: Studies in the philosophy of biology, ed. F. J. Ayala and T. Dobzhansky, pp. 139-61. Macmillan. [JCS]

Campbell, D. T. (1975) On the conflicts between biological and social evolution and between psychology and moral tradition. American Psychologist 30:1103-26. [JCS]

Caporael, L. (2003) Repeated assembly. In: Evolutionary psychology: Alternative approaches, ed. S. J. Scher \& F. Rauscher, pp. 71-89. Kluwer Academic. [aPES]

Caporael, L. R. (1997) The evolution of truly social cognition: The core configurations model. Personality and Social Psychology Review 1:276-98. [LRC]

Caporael, L. R. (2001) Evolutionary psychology: Toward a unifying theory and a hybrid science. Annual Reviews of Psychology 52:607-28. [aPES]

Caporael, L. R. (2014) Evolution, groups, and scaffolded minds. In: Developing scaffolds in evolution, culture, and cognition, ed. L. R. Caporael, J. R. Griesemer, \& W. C. Wimsatt, pp. 57-76. MIT Press. [LRC]

Caporael, L. R. \& Brewer, M. B. (1995) Hierarchical evolutionary theory: There is an alternative, and it's not creationism. Psychological Inquiry 6:31-34. [aPES

Caporael, L. R., Dawes, R. M., Orbell, J. M. \& Van de Kragt, A. J. C. (1989) Selfishness examined: Cooperation in the absence of egoistic incentives. Behavioral and Brain Sciences 12:683-739. [LRC]

Caporael, L. R., Griesemer, J. R. \& Wimsatt, W. C. (2014) Developing scaffolds in evolution, culture, and cognition. MIT Press. [aPES]

Caporael, L. R., Griesiemer J. R. \& Wimsatt W. C. (2014) Developing scaffolds in evolution, culture, and cognition. MIT Press. [LRC]

Cavalli-Sforza, L. L. \& Feldman, M. (1981) Cultural transmission and evolution: A quantitative approach. Princeton University Press. [aPES]

Cavalli-Sforza, L. L., Feldman, M. W., Chen, K. H. \& Dornbusch, S. M. (1982) Theory and observation in cultural transmission. Science 218:19-27. [aPES]

Cederman, L. E. \& Gleditsch, K. S. (2004) Conquest and regime change: An evolutionary model of the spread of democracy and peace. International Studies Quarterly 48:603-29. [MRZ]

Chapais, B. (2008) Primeval kinship: How pair bonding gave birth to human society. Harvard University Press. [DWR]

Cheng, J. T., Tracy, J. L., Foulsham, T., Kingstone, A. \& Henrich, J. (2013) Two ways to the top: Evidence that dominance and prestige are distinct yet viable avenues to social rank and influence. Journal of Personality and Social Psychology 104:103-25. [MM]

Chudek, M., Brosseau-Liard, P., Birch, S. \& Henrich, J. (2013) Culture-gene coevolutionary theory and children's selective social learning. In: Navigating the social world: What infants, children, and other species can teach us, ed. M. R. Banaji \& S. A. Gelman, pp. 181-85. Oxford University Press [aPES]

Chudek, M. \& Henrich, J. (2011) Culture-gene coevolution, norm-psychology and the emergence of human prosociality. Trends in Cognitive Sciences 15(5):218 26. [arPES, MC, MD] 
References/Smaldino: The cultural evolution of emergent group-level traits

Cialdini, R. B. \& Goldstein, N. J. (2004) Social influence: Compliance and conformity. Annual Review of Psychology 55:591-621. [MM]

Claidière, N. \& André, J-B. (2012) The transmission of genes and culture: A questionable analogy. Evolutionary Biology 39:12-24. [aPES]

Clark, H. H. \& Brennan, S. E. (1991) Grounding in communication. In: Perspectives on socially shared cognition, ed. L. B. Resnick, J. M. Levine \& S. D. Teasley, pp. 127-49. American Psychological Association. [aPES]

Cohen, D. (2001) Cultural variation: Considerations and implications. Psychological Bulletin 127:451-71. [rPES]

Cohen, J. E. (2004) Mathematics is biology's next microscope, only better; biology is mathematics' next physics, only better. PLoS Biology 2(12):e439. [aPES]

Collins, B. E. \& Guetzkow, H. (1964) A social psychology of group processes for decision-making. Wiley. [GT]

Colman, A. M. (2003) Cooperation, psychological game theory, and limitations of rationality in social interaction. Behavioral and Brain Science 26:139-53. [TAM]

Colombo, M. (2013) Moving forward (and beyond) the modularity debate: A network perspective. Philosophy of Science 80(3):356-77. [MA]

Cronk, L. \& Gerkey, D. (2007) Kinship and descent. In: The Oxford handbook of evolutionary psychology, ed. R. I. M. Dunbar \& L. Barrett, pp. 463-78. Oxford University Press. [DG]

Cronk, L. \& Leech, B. L. (2013) Meeting at Grand Central: Understanding the social and evolutionary roots of cooperation. Princeton University Press. [DG]

Csibra, G. \& Gergely, G. (2011) Natural pedagogy as evolutionary adaptation. Philosophical Transactions of the Royal Society B 366:1149-57. [aPES]

Cunningham, W. A., Zelazo, P. D., Packer, D. J. \& Van Bavel, J. J. (2007) The iterative reprocessing model: A multilevel framework for attitudes and evaluation. Social Cognition 25:736-60. [aPES]

Dall'Asta, L., Baronchelli, A., Barrat, A. \& Loreto, V. (2006) Non-equalibrium dynamics of language games on complex networks. Physical Review E 74:036105. Available at: http://journals.aps.org/pre/abstract/10.1103/PhysRevE.74.036105 [LS]

Damuth, J. \& Heisler, I. L. (1988) Alternative formulations of multilevel selection. Biology \& Philosophy 3:407-30. [aPES]

Darwin, C. (1871) The descent of man and selection in relation to sex. Appleton Press. [aPES]

Davidson, I. \& Roberts, D. A. (2009) On being alone: The isolation of the Tasmanians. In: Turning points in Australian history, ed. M. Crotty \& D. A. Andrews, pp. 18-31. University of New South Wales Press. [aPES]

Dawkins, R. (1982) Replicators and vehicles. Current Problems in Sociobiology 45:64. [CS]

Dawkins, R. (1989) The extended phenotype. Oxford University Press. [PI]

DeGroot, M. H. (1974) Reaching a consensus. Journal of the American Statistical Association 69(345):118-21. [MA]

Dennett, D. C. (1991) Real patterns. Journal of Philosophy 88:27-51. [rPES]

Dennett, D. C. (2006a) Breaking the spell: Religion as a natural phenomenon. Penguin Books. [aPES]

Dennett, D. C. (2006b) Dan Dennett: Responding to Pastor Rick Warren [Video file]. Available at: http://www.ted.com/talks/dan_dennett_s_response_to_rick_warren.html. [aPES]

Diamond, J. M. (1978) The Tasmanians: The longest isolation, the simplest technology. Nature 273:185-86. [aPES]

Diamond, J. M. (1999) Guns, germs, and steel. W. W. Norton. [aPES]

Dickins, T. E. \& Rahman, Q. (2012) The extended evolutionary synthesis and the role of soft inheritance in evolution. Proceedings of the Royal Society B: Biological Sciences 279(1740):2913-21. [rPES, TCS-P]

Dopfer, K. (2011) 13 Mesoeconomics: A unified approach to systems complexity and evolution. In: Handbook on the economic complexity of technological change, ed. C. Antonelli, pp. 341-56. Edward Elgar Publishing. [TMW]

Dopfer K. (2012) The origins of meso economics. Journal of Evolutionary Economics, 22(1):133-60. doi:10.1007/s00191-011-0218-4. [TMW]

Dunbar, R. I. M. (1993) Coevolution of neocortical size, group size and language in humans. Behavioral and Brain Sciences 16(4):681-735. [LRC]

Dunbar, R. I. M. (1998) The social brain hypothesis. Evolutionary Anthropology 6:178-90. [DWR]

Dunbar, R., Gamble, C. \& Gowlett, J. (2010) Social brain, distributed mind. In: Proceedings of the British Academy 158:3-15. Oxford University Press. [AF]

Durham, W. H. (1991) Coevolution: Genes, culture, and human diversity. Stanford University Press. [arPES, MA]

Durham, W. H. (1992) Applications of evolutionary culture theory. Annual Review of Anthropology 21:331-55. [MA]

Durkheim, E. (1895/1964) The rules of the sociological method, trans. Sarah A. Solovay and John H. Mueller, ed. George E.G. Catlin. Free Press. [DG]

Durrett, R. \& Levin, S. (1994) The importance of being discrete (and spatial). Theoretical Population Biology 46:363-94. [aPES]

Ellickson, R. C. (2009) Order without law: How neighbors settle disputes. Harvard University Press. [ROG]

Ely, R. T. (1901) An introduction to political economy, rev ed. Eaton and Mains. [RAM
Epstein, J. M. (2006) Generative social science. Princeton University Press. [rPES]

Fewell, J. H. \& Page, R. E. (1999) The emergence of division of labour in forced associations of normally solitary ant queens. Evolutionary Ecology Research 1:537-48. [aPES]

Fincher, C. L. \& Thornhill, R. (2012) Parasite-stress promotes in-group assortative sociality: The cases of strong family ties and heightened religiosity. Behavioral and Brain Sciences 352:61-79. [KK]

Fincher, C. L., Thornhill, R., Murray, D. R. \& Schaller, M. (2008) Pathogen prevalence predicts human cross-cultural variability in individualism/collectivism. Proceedings of the Royal Society of London, Series B 275:1279-85. [KK]

Fisher, C. E., Chin, L. \& Klitzman, R. (2010) Defining neuromarketing: Practices and professional challenges. Harvard Review of Psychiatry 18:230-37. [TAM]

Fiske, S. T. (2010) Interpersonal stratification: Status, power, and subordination. In: Handbook of social psychology, 5th ed., ed. S. T. Fiske, D. T. Gilbert \& G. Lindzey, pp. 941-82. Wiley. [MM]

Fletcher, J. A. \& Doebeli, M. (2009) A simple and general explanation for the evolution of altruism. Proceedings of the Royal Society B 276:13-19. [aPES]

Flynn, E.G., Laland, K. N., Kendal, R. L. \& Kendal, J. R. (2013) Developmental niche construction. Developmental Science 16(2):296-313. [AF]

Fodor, J. A. (1974) Special sciences (Or: The disunity of science as a working hypothesis). Synthese 28:97-115. [rPES]

Fothergill, A. \& Berlowitz, V. (2011) Frozen planet: A world beyond imagination. Firefly Books. [aPES]

Fracchia, J. \& Lewontin, R. C. (1999) Does culture evolve? History and Theory 38:52-78. [aPES]

Frank, S. A. (2013) Natural selection. VII. History and interpretation of kin selection theory. Journal of Evolutionary Biology 26:1151-84. [SO]

Fried, M. H. (1967) The evolution of political society: An essay in political anthropology. Random House. [MM]

Fuentes, A. (2013) Cooperation, conflict, and niche construction in the genus homo. In: War, peace, and human nature, ed. D. Fry, pp. 78-94. Oxford University Press. $[\mathrm{AF}]$

Fuentes, A. (in press) Human evolution, niche complexity, and the emergence of a distinctively human imagination. Time and Mind $7(3) \quad[\mathrm{AF}]$

Fuller, R. C. (2001) Spiritual but not religious: Understanding unchurched America. Oxford University Press. [DG]

Galinsky, A. D., Magee, J. C., Gruenfeld, D. H., Whitson, J. A. \& Liljenquist, K. A (2008) Power reduces the press of the situation: Implications for creativity, conformity, and dissonance. Journal of Personality and Social Psychology 95:1450-66. [MM]

Gallotti, M. \& Frith, C. D. (2013) Social cognition in the we-mode. Trends in Cognitive Science 17:160-65. [rPES]

Gamble, C., Gowlett, J. \& Dunbar, R. (2011) The social brain and the shape of the Paleolithic. Cambridge Archeological Journal 21(1):115-36. [AF]

Garbis, C. \& Waern, Y. (1999) Team co-ordination and communication in a rescue command staff: The role of public representations. Le Travail Humain 62:27391. [GT]

Gardner, A. \& Grafen, A. (2009) Capturing the superorganism: A formal theory of group adaptation. Journal of Evolutionary Biology 22(4):659-71. [TCS-P]

Geary, D. C. (2010) Male, female: The evolution of human sex differences, 2 nd ed. American Psychological Association. [MM]

Geertz, C. (1973) The interpretation of cultures: Selected essays. Basic. [RS]

Gerkey, D. (2013) Cooperation in context: Public goods games and post-Soviet collectives in Kamchatka, Russia. Current Anthropology 54:144-76. [rPES]

Gibson, J. J. (1979) The ecological approach to visual perception. Houghton Mifflin. [rPES]

Gigerenzer, G., Todd, P. M. \& the ABC Research Group. (1999) Simple heuristics that make us smart. Oxford University Press. [aPES]

Gilpin, M. E. (1975) The theory of group selection in predator-prey communities. Princeton University Press. [DSW]

Gladwell, M. (2008) Outliers: The story of success. Little, Brown, \& Co. [aPES]

Goldman, E. O. (2002) The spread of Western military models to Ottoman Turkey and Meiji Japan. In: The sources of military change, ed. T. Farrell \& T. Terriff pp. 41-67. Lynne Rienner. [MRZ]

Gong, T. (2009) Computational simulation in evolutionary linguistics: A study on language emergence. Institute of Linguistics, Academia Sinica. [LS]

Gong, T. (2010) Exploring the roles of horizontal, vertical, and oblique transmissions in language evolution. Adaptive Behavior 18(3-4):356-76. [LS]

Gong, T. (2011) Simulating the coevolution of compositionality and word order regularity. Interaction Studies 12(1):63-106. [LS]

Gong, T., Baronchelli, A., Puglisi, A. \& Loreto, V. (2012a) Exploring the roles of complex networks in linguistic categorization. Artificial Life 18(1):107-21. [LS]

Gong, T. \& Shuai, L. (2012) Modelling the coevolution of joint attention and language. Proceedings of the Royal Society B 279:4643-51. [aPES]

Gong, T., Shuai, L., Tamariz, M. \& Jäger, G. (2012b) Studying language change using Price equation and Pólya-urn dynamics. PLoS ONE 7(3):e33171. [LS] 
Goodnight, C. J. (2011) Evolution in metacommunities. Philosophical transactions of the Royal Society of London. Series B, Biological Sciences 366(1569):1401-409. doi:10.1098/rstb.2010.0290 [DSW]

Goodnight, C. J. \& Stevens, L. (1997) Experimental studies of group selection: What do they tell us about group selection in nature? American Naturalist 150:S59S79. [aPES, DSW]

Goodnight, C., Rauch, E., Sayama, H., de Aguiar, M. A. M., Baranger, M. \& BarYam, Y. (2008) Evolution in spatial predator-prey models and the "prudent predator": The inadequacy of steady-state organism fitness and the concept of individual and group selection. Complexity 13(5):23-44. [rPES]

Gopher, D., Itkin-Webman, T., Erev, I., Meyer, J. \& Armony, L. (2000) The effect of shared responsibility and competition in perceptual games: A test of a cognitive game-theoretic extension of signal-detection theory. Journal of Experimental Psychology: Human Perception and Performance 26:325-41. [TAM]

Gottesman, I. I. \& Hanson, D. R. (2006) Human development: Biological and genetic processes. Annual Review of Psychology 56:263-86. [aPES]

Grammich, C., Hadaway, K., Houseal, R., Jones, D. E., Krindatch, A., Stanley, R. \& Taylor, R. H. (2012) 2010 U.S. Religion census: Religious congregations \& membership study. Association of Statisticians of American Religious Bodies. [aPES]

Granovetter, M. S. (1973) The strength of weak ties. American Journal of Sociology 78:1360-80. [rPES]

Greenberger, D., Reiter, W. L. \& Zeilinger, A. (1999) Epistemological and experimental perspectives on quantum physics: Vienna circle institute yearbook. Kluwer Academic. $\quad[\mathrm{TAM}]$

Greif, A. \& Laitin, D. D. (2004) A theory of endogenous institutional change. American Political Science Review 98:633-52. [aPES]

Grim, P., Singer, D. J., Reade, C., \& Fisher, S. (2011) Information dynamics across linked sub-networks: Genes, germs, and memes. In: Proceedings, AAAI Fall Symposium on Complex Systems: Energy, Information, and Intelligence FS-1103, pp. 66-75. AAAI Press. [MA]

Grossman, K. E. \& Grossman, K. (2006) Universality of human social attachment as an adaptive process. In: Attachment and bonding: A new synthesis, ed. C. S. Carter et al., pp. 199-228. MIT Press. [aPES]

Güroğlu, B., van den Bos, W. \& Crone, E. A. (2009) Neural correlates of social decision making and relationships: A developmental perspective. Annals of the New York Academy of Sciences 1167:197-206. [TAM]

Hamilton, W. D. (1964) The genetical evolution of social behaviour. Journal of Theoretical Biology 7:1-52. [DJT]

Hammond, R. A. \& Axelrod, R. (2006) Evolution of contingent altruism when cooperation is expensive. Theoretical Population Biology 69:333-38. [aPES]

Hargadon, A. B. \& Bechky, B. A. (2006) When collections of creatives become creative collectives: A field study of problem solving at work. Organization Science 17(4):484-500. [GT]

Harris, C. B., Barnier, A. J. \& Sutton, J. (2013) Shared encoding and the costs and benefits of collaborative recall. Journal of Experimental Psychology: Learning, Memory and Cognition 39(1):183-95. [GT]

Harris, C. B., Keil, P. G., Sutton, J., Barnier, A. J. \& McIlwain, D. J. F. (2011) "We remember, we forget": Collaborative remembering in older couples. Discourse Processes 48(4):267-303. [GT]

Harris, P. L. (2012) Trusting what you're told: How children learn from others. Harvard University Press. [arPES]

Harton, H. C. \& Bullock, M. (2007) Dynamic social impact: A theory of the origins and evolution of culture. Social and Personality Psychology Compass 1:52140. [MM]

Hegselmann, R. \& Krause, U. (2002) Opinion dynamics and bounded confidence: Models, analysis, and simulation. Journal of Artificial Societies and Social Simulation 5(3):1-33. [MA]

Heine, S. J. (2012) Cultural psychology. 1st and 2nd ed. Norton. [MC]

Heine, S. J., Buchtel, E. E. \& Norenzayan, A. (2008) What do cross-national comparisons of personality traits tell us? The case of conscientiousness. Psychological Science (Wiley-Blackwell) 19(4):309-13. doi: 10.1111/j.14679280.2008.02085.x [KK

Heisler, I. L. \& Damuth, J. (1987) A method for analyzing selection in hierarchically structured populations. American Naturalist 103:582-602. [SO]

Helbing, D. \& Yu, W. (2009) The outbreak of cooperation among success-driven individuals under noisy conditions. Proceedings of the National Academy of Sciences USA 106:3680-85. [aPES]

Henrich, J. (2004a) Cultural group selection, coevolutionary processes and largescale cooperation. Journal of Economic Behavior \& Organization 53:3-35. [aPES, MRZ]

Henrich, J. (2004b) Demography and cultural evolution: How adaptive cultural processes can produce maladaptive losses: The Tasmanian case. American Antiquity 69:197-214. [aPES, MC]

Henrich, J. (2009) The evolution of costly displays, cooperation and religion: Credibility enhancing displays and their implications for cultural evolution. Evolution and Human Behavior 30(4):244-60. [ [MC]
Henrich, J. (2010) The evolution of innovation-enhancing institutions. In: Innovation in cultural systems: Contributions from evolutionary anthropology, ed. M. J O’Brien \& S. J. Shennan, pp. 99-120. MIT Press. [aPES]

Henrich, J. (2011) A cultural species: How culture drove human evolution. Psychological Science Agenda 25(11). http://www.apa.org/science/about/psa/ 2011/11/human-evolution.aspx [AF]

Henrich, J. \& Boyd, R. (2001) Why people punish defectors: Weak conformist transmission can stabilize costly enforcement of norms in cooperative dilemmas. Journal of Theoretical Biology 208:79-89. [aPES]

Henrich, J. \& Boyd, R. (2008) Division of labor, economic specialization, and the evolution of social stratification. Current Anthropology 49(4):715-24. [arPES, MC]

Henrich, J., Boyd, R., Bowles, S., Camerer, C., Fehr, E., Gintis, H., McElreath, R., Alvard, M., Barr, A., Ensminger, J., Henrich, N. S., Hill, K., Gil-White, F., Gurven, M., Marlowe, F. W., Patton, J. Q. \& Tracer, D. (2005) “Economic man" in cross-cultural perspective: Behavioral experiments in 15 small-scale societies. Behavioral and Brain Sciences 28:795-855. [rPES]

Henrich, J., Boyd, R. \& Richerson, P. J. (2008) Five misunderstandings about cultural evolution. Human Nature 19:119-37. [arPES]

Henrich, J., Boyd, R., \& Richerson, P. J. (2012) The puzzle of monogamous marriage. Philosophical Transactions of the Royal Society B: Biological Sciences 367 (1589):657-69. [MC]

Henrich, J. \& Broesch, J. (2011) On the nature of cultural transmission networks: Evidence from Fijian villages for adaptive learning biases. Philosophical Transactions of the Royal Society B 336:1139-48. [MA]

Henrich, J. \& Gil-White, F. J. (2001) The evolution of prestige: Freely conferred deference as a mechanism for enhancing the benefits of cultural transmission. Evolution and Human Behavior 22(3):165-96. [MC]

Herrmann, E., Call, J., Hernández-Lloreda, M. V., Hare, B. \& Tomasello, M. (2007) Humans have evolved specialized skills of social cognition: The cultural intelligence hypothesis. Science 317:1360-66. [aPES]

Hespos, S. J. \& Spelke, E. S. (2004) Conceptual precursors to language. Nature 430:453-56. [rPES]

Hill, K. \& Hurtado, A. M. (2009) Cooperative breeding in South American huntergatherers. Proceedings of the Royal Society B 276:3863-70. [aPES]

Hill, K. R., Walker, R. S. Bozicevic, M., Elder, J., Headland, T., Hewlett, B., Hurtado, A. M., Marlowe, F., Wiessner, P. \& Wood, B. (2011) Co-residence patterns in hunter-gatherer societies show unique human social structure. Science 331:1286-89. [rPES]

Hinde, R. A. (1976a) Interactions, relationships and social structure. McGraw-Hill Book Company. [AF

Hinde, R. A. (1976b) Interactions, relationships and social structure. Man 11:117. [aPES]

Hirst, W. \& Echterhoff, G. (2012) Remembering in conversations: The social sharing and reshaping of memory. Annual Review of Psychology 63:55-79. [GT]

Hirst, W. \& Manier, D. (2008) Towards a psychology of collective memory. Memory 16(3): 183-200. [GT]

Hofbauer, J. \& Sigmund, K (1998) Evolutionary games and population dynamics. Cambridge University Press. [TAM]

Hofstadter, D. R. (2007) I am a strange loop. Basic Books. [rPES]

Hogg, M. A. (2010) Influence and leadership. In: Handbook of social psychology, 5th ed., ed. S. T. Fiske, D. T. Gilbert \& G. Lindzey, pp. 1166-207. Wiley. [MM]

Holden, C. \& Mace, M. (2009) Phylogenetic analysis of the evolution of lactose digestion in adults. Human Biology 81:597-619. [DSW]

Hughes, W. O. H, Oldroyd, B. P., Beekman, M. \& Ratnieks, F. L. W. (2008) Ancestral monogamy shows kin selection is key to the evolution of eusociality. Science 320:1213-16. [PN]

Hull, D. (1988) Science as a process. University of Chicago Press. [LRC]

Hull, D. L. (1980) Individuality and selection. Annual Review of Ecology and Systematics 11:311-32. [rPES]

Hull, D. L. (1981) Units of evolution: A metaphysical essay. In: The philosophy of evolution, ed. R. Jensen \& R. Harré, pp. 23-44. Harvester. [CS]

Hutchins, E. (1995a) How a cockpit remembers its speeds. Cognitive Science 19 (3):265-88. doi:10.1207/s15516709cog1903_1 [ROG]

Hutchins, E. (1995b) Cognition in the wild. MIT Press. [GT, ROG]

Ihara, Y. \& Feldman, M. W. (2004) Cultural niche construction and the evolution of small family size. Theoretical Population Biology 65:105-11. [aPES]

Irons, W. (2001) Religion as a hard-to-fake sign of commitment. In: Evolution and the capacity for commitment, ed. R. Nesse, pp. 292-309. Russell Sage Foundation. [RS]

Isbell, L. \& Young, T. (1993) Social and ecological influences on activity budgets of vervet monkeys, and their implications for group living. Behavioral Ecology and Sociobiology 32:377-85. [DWR]

Jablonka, E. \& Lamb, M. J. (2005) Evolution in four dimensions: Genetic epigenetic, behavioral, and symbolic variation in the history of life. MIT Press. [arPES, AF]

Jackson, M. O. (2008) Social and economic networks. Princeton University Press. [DG] 
Jansen, V. A. A. \& van Baalen, M. (2006) Altruism through beard chromodynamics. Nature 440:663-66. [aPES]

Johnstone, R. (1997) The evolution of animal signals. In: Behavioural ecology: An evolutionary approach, 4th ed., ed. J. Krebs \& N. Davies, pp. 155-78. Blackwell. [RS]

Johnstone, R. A. \& Manica, A. (2011) Evolution of personality differences in leadership. Proceedings of the National Academy of Sciences USA 108:8373-78. [rPES]

Kalenscher, T., Tobler, P. N., Huijbers, W., Daselaar, S. M. \& Pennartz, C. M. (2010) Neural signatures of intransitive preferences. Frontiers in Human Neuroscience 9:49. [TAM]

Kapsalis, E. (2004) Matrilineal kinship and primate behavior. In: Kinship and behavior inprimates, ed. B. Chapais \& C. M. Berman, pp. 153-76. Oxford University Press. [DWR]

Kauffman, S. A. (1971) Articulation of parts explanation in biology and the rational search for them. In: PSA 1970, ed. R. C. Buck \& R. S. Cohen, pp. 257-72. Philosophy of Science Association. [rPES]

Kauffman, S. A. (1993) The origins of order: Self-organization and selection in evolution. Oxford University Press. [rPES]

Keesing, R. (1975) Kin groups and social structure. Holt, Rinehart, and Winston. [DG]

Kelly, D. J., Quinn, P. C., Slater, A. M., Lee, K., Ge, L. \& Pascalis, O. (2007) The other-race effect occurs during infancy: Evidence of perceptual narrowing. Psychological Science 18:1084-89. [rPES]

Kendal, J. (2012) Cultural niche construction and human learning environments: Investigating sociocultural perspectives. Biological Theory 6(3):241-50. [AF]

Kendler, K. S. \& Greenspan, R. J. (2006) The nature of genetic influences on behavior: Lessons from "simpler" organisms. American Journal of Psychiatry 163:1683-94. [aPES]

Kenrick, D. T., Li, N. P. \& Butner, J. (2003) Dynamical evolutionary psychology: Individual decision rules and emergent social norms. Psychological Review 110:3-28. [MM]

Kim, K. \& Markman, A. B. (2006) Differences in fear of isolation as an explanation of cultural differences: Evidence from memory and reasoning. Journal of Experimental Social Psychology 42(3): 350-64. [KK]

Kinzler, K. D., Shutts, K., DeJesus, J. \& Spelke, E. S. (2009) Accent trumps race in guiding children's social preferences. Social Cognition 27:623-34. [rPES]

Kline, M. A. \& Boyd, R. (2010) Population size predicts technological complexity in Oceania. Proceedings of the Royal Society B 277:2559-64. [aPES]

Koella, J. C. (2000) The spatial spread of altruism versus the evolutionary response of egoists. Proceedings of the Royal Society B 267:1979-85. [aPES]

Kuhl, P. K., Williams, K. A., Lacerda, F., Stevens, K. N. \& Lindblom, F. (1992) Linguistic experiences alters phonetic perception in infants by 6 months of age. Science 255:606-608. [rPES]

LaFreniere, P. \& MacDonald, K. (2013) A post-genomic view of behavioral development and adaptation to the environment. Developmental Review 33(2):89102. $[\mathrm{KM}]$

Laland, K. N., Kendal, J. R. \& Brown, G. R. (2007) The niche construction perspective: Implications for evolution and human behaviour. Journal of Evolutionary Psychology 5(1):51-66. [TCS-P]

Laland, K. N., Odling-Smee, J. \& Feldman, M. W. (2000) Niche construction, biological evolution, and cultural change. Behavioral and Brain Sciences 23:13175. $[\mathrm{AF}, \mathrm{aPES}]$

Laland, K. N., Odling-Smee, J. \& Feldman, M. W. (2001) Cultural niche construction and human evolution. Journal of Evolutionary Biology 14:2233. [aPES]

Laland, K. N., Odling-Smee, J., Feldman, M. W. \& Kendal, J. (2009) Conceptual barriers to progress within evolutionary biology. Foundations of Science 14 (3): 195-216. [TCS-P]

Laland, K. N. \& O’Brien, M. J. (2012) Cultural niche construction: An introduction. Biological Theory 6:191-202. [aPES]

Laland, K. N. \& Sterelny, K. (2006) Seven reasons (not) to neglect niche construction. Evolution 60:1751-62. [aPES]

Laland, K. N., Sterlny, K., Odling-Smee, J., Hoppitt, W. \& Uller, T. (2011) Cause and effect in biology revisited: Is Mayr's proximate-ultimate dichotomy still useful? Science 334:1512-16. [aPES]

Lane, D., Maxfield, R. M., Read, D. \& van der Leeuw, S. (2009) From population to organization thinking. In: Complexity perspectives on innovation and social change, ed. D. Lane, D. Pumain, S. van der Leeuw \& G. West, pp. 43-84. Springer-Verlag. [DWR]

Larson, J. R. (2010) In search of synergy in small group performance. Psychology Press. [GT]

Larson, J. R. \& Christensen, C. (1993) Groups as problem-solving units: Toward a new meaning of social cognition. British Journal of Social Psychology 32(1):530. $[\mathrm{GT}]$

Laughlin, P. R. (2011) Group problem solving. Princeton University Press. [GT]

Laughlin, P. R. \& Hollingshead, A. B. (1995) A theory of collective induction. Organizational Behavior and Human Decision Processes 61:94-107. [GT]
Layton, R. \& O'Hara, S. (2010) A comparison of hunter-gatherer and chimpanzee social organization. In: Social brain, distributed mind, ed. R. Dunbar, C. Gamble, \& J. Gowlett, pp. 85-115. Oxford University Press. [LRC]

le Roux, A., Beehner, J. C. \& Bergman, T. J. (2011) Female philopatry and dominance patterns in wild geladas. American Journal of Primatology 73(5):422-30. [DWR

Leaf, M. (2009) Social organization and social theory. University of Illinois Press. [DWR]

Leaf, M. \& Read, D. (2012) The conceptual foundation of human society and thought: Anthropology on a new plane. Lexington Books. [DWR]

Lehrer, K. \& Wagner, C. (1981) Rational consensus in science and society. D. Reidel Publishing. [MA]

Lewontin, R. (1982) Organism and environment. In: Learning, development, and culture ed. H. C. Plotkin, pp. 151-70. Wiley. [aPES]

Lewontin, R. (2000) The triple helix: Gene, organism, and environment. Harvard University Press. [aPES]

Liang, D. W., Moreland, R. \& Argote, L. (1995) Group versus individual training and group performance: The mediating role of transactive memory. Personality and Social Psychology Bulleting 21:384-93. [rPES]

Lindquist, K. A., Wager, T. D., Kober, H., Bliss-Moreau, E. \& Barrett, L. F. (2012 The brain basis of emotion: A meta-analytic review. Behavioral and Brain Sciences 35:121-202. [aPES]

Lion, S. \& van Baalen, M. (2008) Self-structuring in spatial evolutionary ecology. Ecology Letters 11:277-95. [aPES]

Loewenstein, G., Rick, S. \& Cohen, J. (2008) Neuroeconomics. Annual Review of Psychology 59:647-72. [TAM]

Lubell, M. (2013) Governing institutional complexity: The ecology of games framework. Policy Studies Journal 41:537-59. [rPES]

MacDonald, K. (2008) Effortful control, explicit processing and the regulation of human evolved predispositions. Psychological Review 115(4):1012-31. [KM]

MacDonald, K. (2009) Evolution, psychology, and a conflict theory of culture. Evolutionary Psychology 7(2):208-33. [KM]

MacDonald, K. B. (1994) A people that shall dwell alone: Judaism as a group evolutionary strategy. Praeger. [KM]

MacDonald, K. B. (2010) Evolution and a dual processing theory of culture: Applications to moral idealism and political philosophy. Politics and Culture Issue \#1, 2010. http://www.politicsandculture.org/2010/04/29/evolution-and-a-dual-processing-theory-of-culture-applications-to-moral-idealism-and-political-philosophy/ $[\mathrm{KM}]$

MacKillop, J., Amlung, M. T., Wier, L. M., David, S. P., Ray, L. A., Bickel, W. K. \& Sweet, L. H. (2012) The neuroeconomics of nicotine dependence: A preliminary functional magnetic resonance imaging study of delay discounting of monetary and cigarette rewards in smokers. Psychiatry Research 202:20-29. [TAM]

Makowsky, M. D. \& Smaldino, P. E. (2014) The evolution of power and the divergence of cooperative norms. Available at SSRN: http://ssrn.com/ abstract $=2407245 . \quad[\mathrm{rPES}]$

Malone, N. M., Fuentes, A. \& White, F. J. (2012) Variation in the social systems of extant hominoids: Comparative insight into the social behaviour of early hominins. International Journal of Primatology 33(6):1251-77 [AF]

Marshall, J. A. R. (2011) Group selection and kin selection: Formally equivalent approaches. Trends in Ecology and Evolution 26:325-32. [aPES]

Masuda, T. \& Nisbett, R. E. (2001) Attending holistically versus analytically: Comparing the context sensitivity of Japanese and Americans. Journal of Personality and Social Psychology 81:922-34. [aPES]

Maynard Smith, J. (1982) Evolution and the theory of games. Cambridge University Press. [TAM]

Maynard Smith, J. (1998) The origin of altruism. Nature 393:639-40. [DG]

Maynard Smith, J. \& Szathmary, E. (1995) The major transitions in evolution. Oxford University Press. [aPES]

McCain, R. A. (2014) Reframing economics: Economic action as imperfect cooperation. Edward Elgar. [RAM]

McElreath, R. \& Boyd, R. (2007) Mathematical models of social evolution. University of Chicago Press. [aPES]

McElreath, R., Boyd, R. \& Richerson, P. J. (2003) Shared norms and the evolution of ethnic markers. Current Anthropology 44(1):122-29. [aPES, MC]

McKay, R., Harold, J. \& Whitehouse, H. (2013) Catholic guilt? Recall of confession promotes prosocial behavior. Religion, Brain \& Behavior 3(3):201209. [RS]

McNamara, J. M. \& Leimar, O. (2010) Variation and the response to variation as a basis for successful cooperation. Philosophical Transactions of the Royal Society B 365:2627-33. [rPES]

Meade, M. L., Nokes, T. J. \& Morrow, D. G. (2009) Expertise promotes facilitation on a collaborative memory task. Memory 17:38-48. [GT]

Meeks, T. W. \& Jeste, D. V. (2009) Neurobiology of wisdom: A literature overview. Archives of General Psychiatry 66:355-65. [TAM]

Menger, K. (1871/1976) Principles of economics, trans. by James Dingwall \& Bert F Hoselitz. Institute for Humane Studies. [RAM] 
Mesoudi, A. (2007) Biological and cultural evolution: Similar but different. Biological Theory 2:119-223. [aPES]

Mesoudi, A. (2011) Cultural evolution: How Darwinian theory can explain human culture and synthesize the social sciences. University of Chicago Press. [arPES]

Meynen, G. (2013) A neurolaw perspective on psychiatric assessments of criminal responsibility: Decision-making, mental disorder, and the brain. International Journal of Law and Psychiatry 36:93-99. [TAM]

Michod, R. E. (2005) On the transfer of fitness from the cell to the multicellular organism. Biology and Philosophy 20:967-87. [aPES]

Michod, R. E. (2007) Evolution of individuality during the transition from unicellular to multicellular life. Proceedings of the National Academy of Sciences 104:861318. [aPES]

Michod, R. E. \& Nedelcu, A. M. (2003) On the reorganization of fitness during evolutionary transitions in individuality. Integrative and Comparative Biology 43:64-73. [aPES]

Michod, R. E. \& Roze, D. (1997) Transitions in individuality. Proceedings of the Royal Society B 264:853-57. [aPES]

Michod, R. E. \& Roze, D. (2001) Cooperation and conflict in the evolution of multicellularity. Heredity 86:1-7. [aPES]

Mill, J. S. (1987) Principles of political economy. A. M. Kelley. (Reprint of 1909 edition). [RAM $]$

Mojzisch, A. \& Krug, K. (2008) Cells, circuits, and choices: Social influences on perceptual decision making. Cognitive, Affective, \& Behavioral Neuroscience 8:498-508. [TAM]

Moll, H. \& Tomasello, M. (2007) Cooperation and human cognition: The Vygotskian intelligence hypothesis. Philosophical Transactions of the Royal Society B 362:639-48. [aPES]

Moreira, J. A., Pacheco, J. M. \& Santos, F. C. (2013) Evolution of collective action in adaptive social structures. Science Reports 3:1521. [TAM]

Morris, S. (2000) Contagion. Review of Economic Studies 67:57-78. [MA]

Mueller, S. T., Simpkins, B. \& Rasmussen, L. (2010) Incorporating representation when modeling cultural dynamics: Analysis of the bounded influence conjecture. In: Proceedings of the Workshop on Cognitive Social Sciences: Grounding the Social Sciences in the Cognitive Sciences? pp. 29-34, ed. R. Sun. Troy, NY 12180, Rensselaer Polytechnic Institute. Technical Report 2010-RS-0001. [MA]

Murray, D. R., Schaller, M. \& Suedfeld, P. (2013) Pathogens and politics: Further evidence that parasite prevalence predicts authoritarianism. PloS One 8: e62275. [MM]

Murray, D. R., Trudeau, R. \& Schaller, M. (2011) On the origins of cultural differences in conformity: Four tests of the pathogen prevalence hypothesis. Personality and Social Psychology Bulletin 37:318-29. [MM]

Nagel, M. (2010) A mathematical model of democratic elections. Current Research Journal of Social Sciences 2:255-61. [aPES]

Nersessian, N. J. (2006) The cognitive-cultural systems of the research laboratory. Organization Studies 27(1):125-45. [GT]

Nettle, D. (2006) The evolution of personality variation in humans and other animals. The American Psychologist 61(6):622-31. [TCS-P]

Nettle, D. (2007) Individual differences. In: Oxford handbook of evolutionary psychology, ed. R. I. M. Dunbar \& L. Barrett, pp. 479-90. Oxford University Press. [TCS-P]

Newman, M. E. J. (2003) The structure and function of complex networks. SIAM Review 45:167-256. [LS]

Newman, M. E. J. (2010) Networks: An introduction. Oxford University Press [MA]

Nijhout, H. F. (2003) The importance of context in genetics. American Scientist 91:416-23. [aPES]

Nisbett, R. E. \& Cohen, D. (1996) Culture of honor: The psychology of violence in the South. Westview Press. [rPES]

Nisbett, R. E. \& Miyamoto, Y. (2005) The influence of culture: holistic versus analytic perception. Trends in Cognitive Science 9:467-73. [aPES, KK]

Nisbett, R. E., Peng, K., Choi, I. \& Norenzayan, A. (2001) Culture and systems of thought: Holistic versus analytic cognition. Psychological Review 108:291310. [aPES, KK]

Nolin, D. (2011) Kin preference and partner choice: Patrilineal descent and biological kinship in Lamaleran cooperative relationships. Human Nature 22:15676. [DG]

Nonacs, P. (2011a) Monogamy and high relatedness do not preferentially favor the evolution of cooperation. BMC Evolutionary Biology 11:58. [PN]

Nonacs, P. (2011b) Kinship, greenbeards, and runaway social selection in the evolution of social insect cooperation. Proceedings of the National Academy of Sciences, USA 108:10808-15. [PN]

Nonacs, P. \& Kapheim, K. M. (2007) Social heterosis and the maintenance of genetic diversity. Journal of Evolutionary Biology 20:2253-65. [PN]

Nonacs, P. \& Kapheim, K. M. (2008) Social heterosis and the maintenance of genetic diversity at the genome level. Journal of Evolutionary Biology 21:631-35. $[\mathrm{PN}]$
Nonacs, P. \& Kapheim, K. M. (2012) Modeling disease evolution with multilevel selection: HIV as a quasispecies social genome. Journal of Evolutionary Medicine 1:235553. [PN]

Norenzayan, A. (2013) Big gods: How religion transformed cooperation and conflict. Princeton University Press. [MC]

North, D. C. (1990) Institutions, institutional change and economic performance Cambridge University Press. [aPES, MRZ]

Nowak, M. A. (2011) Supercooperators: Altruism, evolution, and why we need each other to succeed. Free Press. [aPES]

Nowak, M. A. \& Sigmund, K. (2005) Evolution of indirect reciprocity. Nature 437:1291-98. [aPES]

O'Gorman, R., Henrich, J. \& Van Vugt, M. (2009) Constraining free riding in public goods games: designated solitary punishers can sustain human cooperation. Proceedings of the Royal Society B: Biological Sciences 276(1655):323-29. doi:10.1098/rspb.2008.1082. [ROG]

O'Gorman, R., Sheldon, K. M. \& Wilson, D. S. (2008a) For the good of the group? Exploring group-level evolutionary adaptations using multilevel selection theory. Group Dynamics: Theory, Research, and Practice 12(1):17-26. doi:10.1037/1089-2699.12.1.17. [ROG]

O’Gorman, R., Wilson, D. S. \& Miller, R. R. (2008b) An evolved cognitive bias for social norms. Evolution and Human Behavior 29(2):71-78. doi:10.1016/j.evolhumbehav.2007.07.002. [ROG, rPES]

Odling-Smee, F. J., Laland, K. N. \& Feldman, M. W. (2003) Niche construction: The neglected process in evolution. Princeton University Press. [arPES]

Okasha, S. (2005) Multilevel selection and the major transitions in evolution. Philosophy of Science 72:1013-25. [aPES]

Okasha, S. (2006) Evolution and the levels of selection. Oxford University Press. [arPES, DG, SO]

Okasha, S. (in press) The relation between kin and multi-level selection: An approach using causal graphs. British Journal for the Philosophy of Science. [SO]

Oyama, S., Griffiths, P. E. \& Gray, R. (2003) Cycles of contingency: Developmental systems and evolution. MIT Press. [AF, DSW]

Oyserman, D., Coon, H. M. \& Kemmelmeier, M. (2002) Rethinking individualism and collectivism: Evaluation of theoretical assumptions and meta-analyses. Psychological Bulletin 128(1):3-72. [KK]

Pacheco, J. M., Traulsen, A., Ohtsuki, H. \& Nowak, M. A. (2008) Repeated games and direct reciprocity under active linking. Journal of Theoretical Biology 250:726-31. [DG]

Pacheco, J. M., Santos, F. C., Souza, M. O. \& Skyrms, B. (2009) Evolutionary dynamics of collective action in $N$-person stag hunt dilemmas. Proceedings of the Royal Society B 276:315-21. [rPES]

Page, R. E. \& Mitchell, S. D. (1991) Self organization and adaptation in insect societies. In: PSA 1990, Vol. 2, ed. A. Fine, M. Forbes \& L. Wessels, pp. 28998. Philosophy of Science Association. [aPES]

Page, S. E. (2007) The difference: How the power of diversity creates better groups, firms, schools, and societies. Princeton University Press. [aPES]

Peng, K. P. \& Nisbett, R. E. (1999) Culture, dialectics, and reasoning about contradiction. Americal Psychologist 54(9):741-54. [KK]

Perc, M. \& Szolnoki, A. (2010) Coevolutionary games: A mini review. Biosystems 99:109-25. [aPES]

Perry, M. (2003) Distributed cognition. In: HCI models, theories, and frameworks: Toward an interdisciplinary science, ed. J. M. Carroll, pp. 193-223. Morgan Kaufmann. [GT]

Pigliucci, M. \& Müller, G. B. eds. (2010) Evolution: The extended synthesis. MIT Press. [TCS-P]

Pinker, S. (2012) The false allure of group selection. Available at: http://www.edge. org/conversation/the-false-allure-of-group-selection [aPES, TCS-P]

Plomin, R. \& Daniels, D. (1987) Why are children in the same family so different from one another? Behavioral and Brain Sciences 10:1-60. [KM]

Plomin, R., DeFries, J. C. \& McLearn, G. E. (2008) Behavioral genetics. Worth. $[\mathrm{KM}]$

Post, C., De Lia, E., DiTomaso, N., Tirpal, T. M. \& Borwankar, R. (2009) Capitalizing on thought diversity for innovation. Research Technology Management 52:14-25. [aPES]

Powell, A., Shennan, S. \& Thomas, M. G. (2009) Late Pleistocene demography and the appearance of modern human behavior. Science 324:1298-301. [aPES]

Price, G. R. (1972) Extension of covariance selection mathematics. Annals of Human Genetics 35:485-90. [aPES]

Puglisi, A., Baronchelli, A. \& Loreto, V. (2008) Cultural route to the emergence of linguistic categories. Proceedings of the National Academy of Sciences of the USA 105(23):7936-40. [LS]

Purzycki, B. \& Sosis, R. (2009) The religious system as adaptive: Cognitive flexibility, public displays, and acceptance. In: The biological evolution of religious mind and behavior, ed. E. Voland \& W. Schiefenhovel, pp. 243-56. SpringerVerlag. [RS]

Range, F. \& Noë, R. (2002) Familiarity and dominance relations among female sooty mangabeys in the Taï National Park. American Journal of Primatology 56:13753. [DWR] 
References/Smaldino: The cultural evolution of emergent group-level traits

Rappaport, R. (1999) Ritual and religion in the making of humanity. Cambridge University Press. [RS]

Read, D. (1984) An algebraic account of the American kinship terminology. Current Anthropology 25(4):417-49. [DWR]

Read, D. (2001) What is kinship? In: The cultural analysis of kinship: The legacy of David Schneider and its implications for anthropological relativism, ed. R. Feinberg \& M. Ottenheimer, pp. 78-117. University of Illinois Press. [DWR]

Read, D. (2006) Tasmanian knowledge and skill: Maladaptive imitation or adequate technology? American Antiquity 71:164-84. [aPES]

Read, D. (2007) Kinship theory: A paradigm shift. Ethnology 46(4):329-64. [DWR]

Read, D. (2010) The algebraic logic of kinship terminology structure. Behavioral and Brain Sciences 33(5):399-400. [DWR]

Read, D. (2012) How culture makes us human: Primate evolution and the formation of human societies. Left Coast Press. [DWR]

Read, D. (2013) A new approach to forming a typology of kinship terminology systems: From Morgan and Murdock to the present. Structure and Dynamics 6 (1). http://www.escholarship.org/uc/item/0ss6j8sh [DWR]

Read, D., Lane, D. \& van der Leeuw, S. (2009) The innovation innovation. In: Complexity perspectives in innovation and social change, ed. D. Lane, D. Pumain, S. van der Leeuw \& G. West, pp. 43-84. Springer-Verlag. [DWR]

Read, D., Leaf, M. \& Fischer, M. D. (2013) What are kinship terminologies, and why do we care? A computational approach to analyzing symbolic domains. Social Science Computer Review 31(1):16-44. [DWR]

Read, L. (1958) I, pencil: My family tree as told to Leonard E. Read. In: The Freeman; reprinted by The Library of Economics and Liberty, found on 9/3/12 at http://www.econlib.org/library/Essays/rdPncll.html. [aPES]

Reeve, H. K. \& Hölldobler, B. (2007) The emergence of a superorganism through intergroup competition. PNAS 104:9736-40. [aPES]

Resnick, L. B., Levine, J. M. \& Teasdale, S. D., eds. (1991) Perspectives on socially shared cognition. American Psychological Association. [MM]

Ricardo, D. (1817) On the principles of political economy and taxation, 1st ed. John Murray. $\quad[\mathrm{PI}]$

Richerson, P. J. (2012) Comment on Steven Pinker's Edge essay. Available at: http://socialevolutionforum.com/2012/06/28/peter-j-richerson-comment-onsteven-pinkers-edge-essay/ [TCS-P]

Richerson, P. J. \& Boyd, R. (1998) The evolution of human ultra-sociality. In: Ideology, warfare, and indoctrinability, ed. I. Eibl-Eibisfeldt \& F. Slater, pp. 71-95. Bergahn Books. [aPES]

Richerson, P. J. \& Boyd, R. (2005) Not by genes alone: How culture transformed human evolution. University of Chicago Press. [arPES, AF, TCS-P, TD]

Richerson, P. J. \& Henrich, J. (2012) Tribal social instincts and the cultural evolution of institutions to solve collective action problems. Cliodynamics 3:38-80. [aPES]

Ridley, M. (2010) The rational optimist: How prosperity evolves. HarperCollins. [aPES]

Roccas, S. \& Brewer, M. B. (2002) Social identity complexity. Personality and Social Psychology Review 6:88-106. [rPES]

Sanfey, A. G. (2007) Social decision-making: Insights from game theory and neuroscience. Science 318:598-602. [TAM]

Saxe, R. \& Kanwisher, N. (2003) People thinking about thinking people: The role of the temporo-parietal junction in "theory of mind." NeuroImage 19:1835-42. [TAM]

Schaller, M. (2011) The behavioural immune system and the psychology of human sociality. Philosophical Transactions of the Royal Society of London Series BBiological Sciences 366:3418-26. [KK]

Schaller, M. \& Crandall, C. S., eds. (2003) The psychological foundations of culture. Erlbaum. [MM]

Schank, J. C. (2001) Beyond reductionism: Refocusing on the individual with individual-based modeling. Complexity 6(3):33-40. [rPES]

Scott-Phillips, T. C., Dickins, T. E. \& West, S. A. (2011) Evolutionary theory and the ultimate-proximate distinction in the human behavioral sciences. Perspectives on Psychological Science 6(1):38-47. [TCS-P]

Seeley, T. D. (2010) Honeybee democracy. Princeton University Press. [DSW, rPES]

Semmann, D., Krambeck, H. \& Milinski, M. (2005) Reputation is valuable within and outside one's own social group. Behavioral Ecology and Sociobiology 57:611-16. [KM]

Simon, B. (2008) A stochastic model of evolutionary dynamics with deterministic large population asymptotics. Journal of Theoretical Biology 254:719-30. [MD]

Simon, B., Fletcher, J. A. \& Doebeli, M. (2013) Towards a general theory of group selection. Evolution 67:1561-72. [rPES, MD]

Simonton, D. K. (1999) Talent and its development: An emergenic and epigenetic model. Psychological Review 106:435-57. [aPES]

Smaldino, P. E. \& Lubell, M. (2011) An institutional mechanism for assortment in an ecology of games. PLoS ONE 6:e23019. [arPES]
Smaldino, P. E. \& Lubell, M. (2014) Institutions and cooperation in an ecology of games. Artificial Life 20:207-21. [arPES]

Smaldino, P. E., Newson, L., Schank, J. C. \& Richerson, P. J. (2013a) Simulating the evolution of the human family: Cooperative breeding increases in harsh environments. PLoS ONE 8(11):e80753. [arPES]

Smaldino, P. E., Pickett, C. L., Sherman, J. W. \& Schank, J. C. (2012) An agentbased model of social identity dynamics. Journal of Artificial Societies and Social Simulation 15(4):7. [CS]

Smaldino, P. E. \& Richerson, P. J. (2012) The origins of options. Frontiers in Neuroscience 6:50. [arPES]

Smaldino, P. E. \& Schank, J. C. (2012a) Movement patterns, social dynamics, and the evolution of cooperation. Theoretical Population Biology 82:48-58. [aPES]

Smaldino, P. E. \& Schank, J. C. (2012b) Invariants of human emotion. Behavioral and Brain Sciences 35:164. [aPES]

Smaldino, P. E., Schank, J. C. \& McElreath, R. (2013b) Increased costs of cooperation help cooperators in the long run. The American Naturalist 181 (4):451-63. [arPES, CS]

Smith, A. (1994) The wealth of nations [Reprint of 1909 edition]. The Modern Library. [RAM]

Smith, E. A. (2005) Making it real: Interpreting economic experiments. Behavioral and Brain Sciences 28:832-33. [KM]

Sober, D. (1984) The nature of selection: Evolutionary theory in philosophical focus. MIT Press. [rPES]

Sober, E. \& Wilson, D. S. (1998) Unto others: The evolution and psychology of unselfish behavior. Harvard University Press. [DJT]

Soltis, J., Boyd, R. \& Richerson, P. J. (1995) Can group-functional behaviors evolve by cultural group selection? An empirical test. Current Anthropology 36:47394. [aPES]

Sosis, R. (2003) Why aren't we all Hutterites? Costly signaling and religious behavior Human Nature 14:91-127. [RS]

Sosis, R. (2009) The adaptationist-byproduct debate on the evolution of religion: Five misunderstandings of the adaptationist program. Journal of Cognition and Culture 9:315-32. [RS]

Sosis, R. (2011) Why sacred lands are not indivisible: The cognitive foundations of sacralizing land. Journal of Terrorism Research 2:17-44. [RS]

Sosis, R. \& Ruffle, B. (2003) Religious ritual and cooperation: Testing for a relationship on Israeli religious and secular kibbutzim. Current Anthropology 44:713-22. [RS]

Spector, M. (1972) Methodological foundations of relativistic mechanics. University of Notre Dame Press. [TAM]

Sperber, D. (1996) Explaining culture: A naturalistic approach. Blackwell. [TCS-P]

Sperber, D. \& Claidière, N. (2006) Why modeling cultural evolution is still such a challenge. Biological Theory 1:20-22. [aPES]

Sperber, D. \& Hirschfeld, L. A. (2004) The cognitive foundations of cultural stability and diversity. Trends in Cognitive Sciences 8(1):40-46. [TCS-P]

Spoor, J. \& Williams, K. D. (2007) The evolution of an ostracism detection system. In: Evolution and the social mind: evolutionary psychology and social cognition, ed. J. P. Forgas, W. von Hippel \& M. G. Haselton, Psychology Press. [ROG]

Stark, R. (1996) The rise of Christianity. Princeton University Press. [DG]

Steiner, I. D. (1966) Models for inferring relationships between group size and potential group productivity. Behavioral Science 11:273-83. [GT]

Sterelny, K. (1996) The return of the group. Philosophy of Science 63:562-84 [DG]

Sterelny, K. (2012) The evolved apprentice: How evolution made humans unique. MIT Press. [AF, GT]

Sternberg, R. J. (1998) Cognitive mechanisms in human creativity: Is variation blind or sighted? The Journal of Creative Behavior 32:159-76. [JCS]

Strogatz, S. H. \& Stewart, I. (1993) Coupled oscillators and biological synchronization. Scientific American 269(6):68-74. [rPES]

Sumpter, D. J. T. (2006) The principles of collective animal behaviour. Philosophical Transactions of the Royal Society B 361:5-22. [aPES, PI]

Surowiecki, J. (2004) The wisdom of crowds. Anchor Books and Doubleday. [aPES, GT]

Sutton, J. (2010) Exograms and interdisciplinarity: History, the extended mind, and the civilizing process. In: The Extended Mind, ed. R. Menary, pp. 189-225. MIT Press. [GT]

Sutton, J. (2013) Collaboration and skill in the evolution of human cognition. Biological Theory 8(1):28-36. [GT]

Sutton, J., Harris, C. B., Keil, P. G. \& Barnier, A. J. (2010) The psychology of memory, extended cognition, and socially distributed remembering. Phenomenology and the Cognitive Sciences 9(4):521-60. [GT]

Tardos, E. \& Vazirani, V. V. (2007) Basic solution concepts and computational issues In: Algorithmic game theory, ed. N. Nisan, T. Roughgarden, E. Tardos \& V. V. Vazirani, pp. 3-26. Cambridge University Press. [RAM]

Theiner, G. (2013) Transactive memory systems: A mechanistic analysis of emergent group memory. Review of Philosophy and Psychology 4(1):65-89. [GT] 
References/Smaldino: The cultural evolution of emergent group-level traits

Theiner, G. \& O'Connor, T. (2010) The emergence of group cognition. In: Emergence in science and philosophy, ed. A. Corradini \& T. O'Connor, pp. 78-117. Routledge. [GT]

Tomasello, M. (2009) Why we cooperate. MIT Press. [aPES]

Tomasello, M., Carpenter, M., Call, J., Behne, T. \& Moll, H. (2005) Understanding and sharing intentions: The origins of cultural cognition. Behavioral and Brain Sciences 28(05):675-735. doi:10.1017/S0140525X05000129. [aPES, ROG]

Tooby, J. \& Cosmides, L. (1992) The psychological foundations of culture. In: The adapted mind: Evolutionary psychology and the evolution of culture. ed. J. H. Barkow, L. Cosmides \& J. Tooby, pp. 19-136. Oxford University Press. [aPES]

Tribble, E. B. (2005) Distributing cognition in the globe. Shakespeare Quarterly $56: 135-55 . \quad[\mathrm{GT}]$

Trivers, R. (1971) The evolution of reciprocal altruism. Quarterly Review of Biology 46:35-57. [aPES]

Vaesen, K. (2012) Cumulative cultural evolution and demography. PLoS ONE 7: e40989. [aPES]

Vallacher, R. R. \& Nowak, A. (2007) Dynamical social psychology: Finding order in the flow of human experience. In: Social psychology: Handbook of basic principles. ed. W. A. Kruglanski \& E. T. Higgins, 2nd ed., pp. 734-58. Guilford Press. [rPES]

van den Bergh, J. C. J. M. \& Gowdy, J. M. (2009) A group selection perspective on economic behavior, institutions and organizations. Journal of Economic Behavior \& Organization 72:1-20. [aPES]

van Ijzendoorn, M. H., Bakermans-Kranenburg, M. J. \& Sagi-Schwartz, A. (2006) Attachment across diverse sociocultural contexts: The limits of universality. In: Parenting beliefs, behaviors, and parent-child relations: A cross-cultural perspective, ed. K. H. Rubin, pp. 107-42. Psychology Press. [aPES]

Van Vugt, M., Hogan, R. \& Kaiser, R. B. (2008) Leadership, followership, and evolution. American Psychologist 63:182-96. [MM]

Vega-Redondo, F. (2007) Complex social networks. Cambridge University Press. [MA]

von Neumann J. \& Morgenstern, O. (1946) Theory of games and economic behavior. Princeton University Press. [TAM]

Wade, M. J. (1978) A critical review of models of group selection. Quarterly Review of Biology 53:101-14. [arPES]

Wade, M. J., Wilson, D. S., Goodnight, C., Taylor, D., Bar-Yam, Y., de Aguiar, M. A. M., Stacey, B., Werfel, J., Hoelzer, G. A., Brodie, E. D., Fields, P., Breden, F., Linksvayer, T. A., Fletcher, J. A., Richerson, P. J., Bever, J. D., Van Dyken, J D. \& Zee, P. (2010) Multilevel and kin selection in a connected world. Nature 463:E8-E10. [aPES]

Wagenhals, L. W. \& Alexander, H. L. (2001) Modeling effects-based operations in support of war games. In: Enabling technology for simulation science, Proceedings of SPIE, Vol. 4367. ed. V. A. F. Sisti \& D. A. Trevisani, pp. 365-76. Society of Photo-Optical Instrumentation Engineers [JCS]

Waring, T. M. (2012) Cooperation dynamics in a multi-ethnic society: A case study from Tamil Nadu. Current Anthropology 53(5):642-49. [aPES]

Wasserman, S. \& Faust, K. (1994) Social network analysis: Methods and applications. Cambridge University Press. [DG, MA]

Watts, D. J. (1999) Small worlds. Princeton University Press. [LS]

Weber, E. U. \& Hsee, C. K. (2000) Culture and individual judgment and decision making. Psychologie Appliquee - Revue Internationale (Applied Psychology An International Review- 49(1):32-61. [KK]

Wegner, D. M. (1986) Transactive memory: A contemporary analysis of the group mind. In: Theories of group behavior, pp. 185-208. Springer. [GT]

Weldon, M. S. \& Bellinger, K. D. (1997) Collective memory: Collaborative and individual processes in remembering. Journal of Experimental Psychology: Learning, Memory, and Cognition 23:1160-75. [GT]

Werner, C. (2009) Bride abduction in post-Soviet Central Asia: Marking a shift towards patriarchy through local discourses of shame and tradition. Journal of the Royal Anthropological Institute 15:314-31. [rPES]

West, S. A., El Mouden, C. \& Gardner, A. (2011) Sixteen common misconceptions about the evolution of cooperation in humans. Evolution and Human Behavior 32(4):231-62. [TCS-P]

White, O. (1965) Parliament of a thousand tribes: A study of New Guinea. Heinemann. [aPES]
Whiten, A. \& Erdal, D. (2012) The human socio-cognitive niche and its evolutionary origins. Philosophical Transactions of the Royal Society B 367:2119-29. [AF]

Whiten, A., Hinde, R. A., Laland, K. N. \& Stringer, C. B. (2011) Culture evolves. Philosophical Transactions of the Royal Society B 366:938-48. [aPES]

Wild, G., Gardner, A. \& West, S. A. (2009) Adaptation and the evolution of parasite virulence in a connected world. Nature 459:983-86. [aPES]

Wildman, W. J. \& Sosis, R. (2011) Stability of groups with costly beliefs and practices Journal of Artificial Societies and Social Stimulation 14(3):6. [RS]

Williams, G. C. (1966) Adaptation and natural selection. Princeton University Press. [aPES]

Wilson, D. S. (1975) A theory of group selection. Proceedings of the National Academy of Sciences 72:143-46. [arPES, DG]

Wilson, D. S. (1992) The effect of complex interactions on variation between units of a metacommunity, with implications for biodiversity and higher levels of selection. Ecology 73:1984-2000. [DSW]

Wilson, D. S. (1997) Altruism and organism: Disentangling the themes of multilevel selection theory. American Naturalist 150:S122-34. [aPES, DSW]

Wilson, D. S. (2002) Darwin's cathedral: evolution, religion, and the nature of society. University of Chicago Press. [aPES, ROG]

Wilson, D. S. \& Dugatkin, L. A. (1997) Group selection and assortative interactions American Naturalist 149:336-51. [aPES]

Wilson, D. S. \& O'Gorman, R. (2003) Emotions and actions associated with norm-breaking events. Human Nature 14(3):277-304. doi:10.1007/s12110-0031007-z. [ROG]

Wilson, D. S. \& Sober, E. (1994) Reintroducing group selection to the human behavioral sciences. Behavioral and Brain Sciences 17(4):585-607. [aPES, TCS-P]

Wilson, D. S. \& Wilson, E. O. (2007) Rethinking the theoretical foundation of sociobiology. The Quarterly Review of Biology 82(4):327-48. [aPES, TCS-P]

Wimsatt, W. C. (1974) Complexity and organization. In: PSA 1972, ed. K. Schaffner \& R. S. Cohen, pp. 67-86. Philosophy of Science Association. [aPES, RAM]

Wimsatt, W. C. (1986) Forms of aggregativity. In: Human nature and natural knowledge, ed. M. G. Grene, A. Donagan, A. N. Perovich \& M. V. Wedin, pp. 259-91. Reidel. [GT]

Wimsatt, W. C. (1997) Aggregativity: Reductive heuristics for finding emergence. Philosophy of Science 64(4):S372-84. [arPES, JCS, CS]

Wimsatt, W. C. (2000) Emergence as non-aggregativity and the biases of reductionisms. Foundations of Science 5(3):269-97. [CS]

Wimsatt, W. C. (2006) Aggregate, composed, and evolved systems: Reductionistic heuristics as means to more holistic theories. Biology \& Philosophy 21:667702. [arPES]

Wimsatt, W. C. \& Griesemer, J. R. (2007) Reproducing entrenchments to scaffold culture: The central role of development in cultural evolution. In: Integrating evolution and development: From theory to practice, ed. R. Sansom \& R. Brandon, pp. 227-323. MIT Press. [aPES]

Wolf, M. \& McNamara, J. M. (2013) Adaptive between-individual differences in social competence. Trends in Ecology \& Evolution 28:253-54. [rPES]

Wood, W. (2000) Attitude change: Persuasion and social influence. Annual Review of Psychology 51:539-70. [MM]

Wood, W. \& Eagly, A. H. (2010) Gender. In: Handbook of social psychology, 5th ed., ed. S. T. Fiske, D. T. Gilbert \& G. Lindzey, pp. 629-67. Wiley. [MM]

Woolley, A. W., Chabris, C. F., Pentland, A., Hashmi, N. \& Malone, T. W. (2010) Evidence for a collective intelligence factor in the performance of human groups. Science 330:686-88. [aPES]

Wray, M. K., Mattila, H. R. \& Seeley, T. D. (2011) Collective personalities in honeybee colonies are linked to colony fitness. Animal Behaviour 81:559-68. $[\mathrm{PN}]$

Wynne-Edwards, V. C. (1962) Animal dispersion in relation to social behavior. Oliver \& Boyd. [aPES]

Xygalatas, D. (2013) Effects of religious setting on cooperative behavior: A case study from Mauritus. Religion, Brain \& Behavior 3(2):91-102. [RS]

Young, H. P. (1998) Individual strategy and social structure: An evolutionary theory of institutions. Princeton University Press. [MA, aPES]

Zeeman, E. C. (1980) Population dynamics from game theory. Global Theory of Dynamical Systems/Lecture Notes in Mathematics 819:471-97. [TAM]

Zollman, K. J. (2013) Network epistemology: Communication in epistemic communities. Philosophy Compass 8(1):15-27. [MA] 